\title{
SHARP INEQUALITIES, THE FUNCTIONAL DETERMINANT, AND THE COMPLEMENTARY SERIES
}

\author{
THOMAS P. BRANSON
}

\begin{abstract}
Results in the spectral theory of differential operators, and recent results on conformally covariant differential operators and on sharp inequalities, are combined in a study of functional determinants of natural differential operators. The setting is that of compact Riemannian manifolds. We concentrate especially on the conformally flat case, and obtain formulas in dimensions 2,4 , and 6 for the functional determinants of operators which are well behaved under conformal change of metric. The two-dimensional formulas are due to Polyakov, and the four-dimensional formulas to Branson and Ørsted; the method is sufficiently streamlined here that we are able to present the sixdimensional case for the first time. In particular, we solve the extremal problems for the functional determinants of the conformal Laplacian and of the square of the Dirac operator on $S^{2}$, and in the standard conformal classes on $S^{4}$ and $S^{6}$. The $S^{2}$ results are due to Onofri, and the $S^{4}$ results to Branson, Chang, and Yang; the $S^{6}$ results are presented for the first time here. Recent results of Graham, Jenne, Mason, and Sparling on conformally covariant differential operators, and of Beckner on sharp Sobolev and Moser-Trudinger type inequalities, are used in an essential way, as are a computation of the spectra of intertwining operators for the complementary series of $\mathrm{SO}_{0}(m+1,1)$, and the precise dependence of all computations on the dimension. In the process of solving the extremal problem on $S^{6}$, we are forced to derive a new and delicate conformally covariant sharp inequality, essentially a covariant form of the Sobolev embedding $L_{1}^{2}\left(S^{6}\right) \hookrightarrow L^{3}\left(S^{6}\right)$ for section spaces of trace free symmetric two-tensors.
\end{abstract}

\section{INTRODUCTION}

Some very recent work in analysis and geometry has revealed strong new connections among fields which, while never completely separate, have at least been studied in very different ways. Part of the stimulus for this has been physical string theory, which led in the last decade to a fresh look at Riemann surfaces, always a meeting ground for different disciplines in analysis. In this paper, we would like to clarify some of these connections as they have manifested themselves in the study of string theoretic principles in higher $(>2)$ dimensions. Broadly speaking, the fields in question are:

(I) the spectral theory of differential operators;

(II) conformal geometry; and

Received by the editors June 30, 1992 and, in revised form, July 16, 1994.

1991 Mathematics Subject Classification. Primary 58G25; Secondary 22E46, 26 D10.

Partially supported by the Danish Research Council. 
(III) sharp inequalities.

In this discussion, we work in the category of compact Riemannian manifolds. Under the heading (I) are such tools as the heat kernel expansion, index theory, and functional determinants. Two of the major goals involve relating geometry to spectral data. In the isospectral problem, one tries to show that the spectrum of some natural differential operator (for example, the Laplacian $\Delta$ ) determines the geometry (Riemannian metric), or at least that there is a very small set of metrics with the same spectrum. In the uniformization problem, one tries to use some spectral invariant $\mathscr{S}$ to "drive" the metric to a uniform state (usually one of constant curvature in some sense), either by solving an extremal problem for $\mathscr{S}$ as a functional on the space of metrics, or by setting up a "heat flow" (parabolic equation) which will produce the uniform state at time infinity. Under the heading (II), the main tools are conformally invariant differential operators. (III) is a hard analytic topic; among the major tools relevant here is symmetrization and the use of symmetric decreasing rearrangement to "improve" the value of functionals like those mentioned in the discussion of (I) above. "Sharp" inequalities are those with best constants and extremals given. Geometrically, a sharp inequality can usually be viewed as an invariant quantity, i.e., the quantity asserted to be nonnegative. Sharpness, not to mention the applicability of symmetrization in the proof, are intimately connected to the geometric invariance exhibited by the eventual inequality. More functional analytically, a sharp inequality can often be viewed as a norm computation for an embedding of Banach spaces, the prototypical example being the Sobolev Embedding Theorem. Both of these viewpoints will be useful here.

The more or less straightforward connections are (I) $\leftrightarrow$ (II) and (II) $\leftrightarrow$ (III); the link (I) $\leftrightarrow$ (III) has been somewhat mysterious. For example, one would like a reason for the fact that Onofri's analysis of the extremal problem for the functional determinant $\operatorname{det} \Delta$ on the sphere $S^{2}$ leads directly to the MoserTrudinger inequality, other than that both problems are extremely natural. A main goal of this paper, aside from showing that similar phenomena occur in higher dimensions, is to show that these phenomena should be expected, i.e., are to some extent predictable from the geometric formulation of the problems. In other words, we would like to make the link (I) $\leftrightarrow$ (III) explicit.

A curious additional connection is that between (III) and the theory of the complementary series of the Lie group $\mathrm{SO}_{0}(m+1,1)$; we make this completely explicit in Section 3 of this paper. It has been remarked that, with respect to the problem of determining the unitary dual of a semisimple Lie group, the complementary series is an anomalous sort of object, not really fitting into any framework that does much good in the study of unitary representations in general. For this reason, complementary series representations have even acquired a reputation as being somewhat "useless". (According to [V, p. 17], "One hopes not to need them for most harmonic analysis problems.") It is, therefore, ironic that they are so important in the present treatment, and more so because they are useful for the same reason that they have been thought useless: the invariant inner product is a "strange" one, obtained from the $L^{2}$ inner product $(\cdot, \cdot)$ by insertion of a pseudo-differential intertwining operator $A$ to form $(\cdot, A \cdot)$. These $A$ are actually elliptic operators defining invariant versions of the inner products in the Sobolev spaces $L_{\nu}^{2}$ (meaning $L^{2}$, with $\nu$ derivatives in $L^{2}$, 
$\nu \in \mathbb{R})$. Otherwise said, they are invariant Banach isomorphisms from $L_{\nu}^{2}$ to its dual $L_{-\nu}^{2}$; for a discrete set of $\nu$, they are actually differential operators, and in fact the realizations on the standard sphere $S^{m}$ of conformally covariant differential operators that exist for general Riemannian metrics. Furthermore, the spectra of these intertwining operators are explicitly computable from the intertwining relation. We return to this theme in Section 7 to derive an essentially new estimate crucial to the extremal problem for the functional determinant on $S^{6}$; here we need not only the complementary series representations from the spherical principal series (principal series representations carried by spaces of scalar valued functions), but also a tensor valued complementary series. In a way, the complementary series is the central object in this investigation; indeed, we have just touched upon links to all the other ideas in this circle (including (I-III)) in discussing it.

In describing the results of this work, we shall stay on a not-too-technical level for the remainder of the introduction (just referring ahead for definitions and clarification). We continue the study of the functional determinant in higher $(>2)$ dimensions begun in [BØ3, BCY], and derive new formulas for functional determinants of operators with certain nice conformal properties, in particular for the conformal Laplacian (or Yamabe operator) $Y$ and the square $\nabla^{2}$ of the Dirac operator, on conformally flat manifolds of dimension 6 . One motivation for going to dimension six is the search for a pattern in the extremal problem for the functional determinant. In a fundamental paper [On], Onofri showed that as a functional on the space of volume $4 \pi$ Riemannian metrics on the Riemann sphere $S^{2}$, the functional determinant det $\Delta$ of the Laplacian is maximized exactly at the standard metric $g_{0}$ and its transforms under the Möbius group of $S^{2}$. His method was as follows: (1) prove the statement replacing "Riemannian metrics" by "metrics in the standard conformal class $\left[g_{0}\right]:=\left\{g_{\omega}=e^{2 \omega} g \mid \omega \in C^{\infty}\left(S^{2}\right)\right\}$." This is done by computing a quotient $\left(\operatorname{det} \Delta_{\omega}\right) /\left(\operatorname{det} \Delta_{0}\right)$, where $\Delta_{\omega}$ is the Laplacian in the metric $g_{\omega}$, and then noticing that the resulting quantity is exactly that asserted to be nonpositive by the celebrated Moser-Trudinger inequality. This inequality may be viewed as the norm computation for the embedding of the Sobolev class $L_{1}^{2}$ into the Orlicz class $e^{L}$. (2) Then note that any Riemannian metric on $S^{2}$ is the pullback of some $g_{\omega}$ under a diffeomorphism $\varphi \in \operatorname{Diffeo}\left(S^{2}\right)$. Since spectral invariants of $\Delta$ or of any other natural differential operator are also diffeomorphism invariants, the result follows; the spectral invariants involved here are, of course, $\operatorname{det} \Delta$ and the volume (which appears in the spectral asymptotics of $\Delta$ ). Similar results hold for the Laplacians of higher spin bundles; in particular the spin Laplacian, i.e., the square $\nabla^{2}$ of the Dirac operator $\not$, though one important dissimilarity is that det $\nabla^{2}$ is minimized in the standard geometry.

The first thing to go radically wrong with Onofri's argument when we go up in dimension is this second step: generalizing the Möbius group to the conformal group $\operatorname{ctran}\left(S^{m}, g_{0}\right)$, up to covering a copy of $\mathrm{SO}_{0}(m+1,1)$, we get an extremely large space of metrics, even after deflation by the infinite dimensional groups of diffeomorphisms and of conformal changes of metric. To be somewhat more precise, the cone $\mathscr{G}$ of Riemannian metrics is acted upon by the group $\operatorname{Diffeo}\left(S^{m}\right) \ltimes C_{+}^{\infty}\left(S^{m}\right)$, where $\ltimes$ is semidirect product, and the action of $e^{\omega} \in C_{+}^{\infty}\left(S^{m}\right)$ is $g \mapsto e^{2 \omega} g$. The product is semidirect because 
diffeomorphisms pull back conformal factors $e^{\omega}$. If $m=2$, the orbit space $\mathscr{G} / \operatorname{Diffeo}\left(S^{m}\right) \ltimes C_{+}^{\infty}\left(S^{m}\right)$ is a single point, but if $m>2$, it is infinite dimensional and quite complicated structurally. The upshot is that, in higher dimensions, we must stay within a given conformal class, at least at first, in looking for analogues of Onofri's Theorem and other fundamental string theoretic principles. Almost no work has been done on the question of cutting across conformal classes, either for the extremal problem or the closely related isospectral problem.

There are still more unpleasant facts to be faced in higher dimension: much as we like the ordinary Laplacian $\Delta$ (for example, for its topological significance), it simply does not have the good conformal behavior necessary to get a Polyakov formula for the conformal variation of its determinant. Nature strongly prefers that we compute the determinant of a conformally covariant differential operator, or at least a positive integral power of such. The Dirac operator $\not$ is conformally covariant, so we can always consider $\nabla^{2}$. There is also a Laplacian on functions, the conformal Laplacian

$$
Y:=\Delta+\frac{m-2}{4(m-1)} \tau,
$$

where $\tau$ is the scalar curvature, which has nice conformal properties, and which specializes to $\Delta$ in dimension two.

At this point one would like to examine the situation in dimension $m=3$. However, the functional determinant is quite rigid conformally in odd dimensions, and so at least for the problem we are looking at, the next dimension is $m=4$. Here Branson and Ørsted [BØ3] computed quotients of the form

$$
\left(\operatorname{det} A_{\omega}\right) /\left(\operatorname{det} A_{0}\right)
$$

for reasonably general operators $A$ and background metrics $g_{0}$. (In particular, conformal flatness was not assumed.) As above, the subscript $\omega$ indicates that we evaluate in the metric $g_{\omega}=e^{2 \omega} g_{0}$. Later, Branson, Chang, and Yang [BCY] attacked the isospectral and extremal problems related to these formulas. One result of this latter work is that something survives of the Onofri reasoning: consider the extremal problems for det $Y_{\omega}$ and det $\nabla_{\omega}^{2}$ in the standard conformal class $\left[g_{0}\right]$ on $S^{4}$. What emerges from the quantities $(0.1)$ is now a linear combination of two norm computations: one for the exponential class embedding $L_{2}^{2} \hookrightarrow e^{L}$, and another for the "ordinary" borderline Sobolev embedding $L_{1}^{2} \hookrightarrow L^{4}$. The latter inequality is that involved in the Yamabe problem, and in fact it is quite fruitful to think of it in this way. The former inequality is the four-dimensional version of a generalized Moser-Trudinger inequality due to Beckner [Bec]. The "miracle" that occurs is that in this linear combination, the signs of the two coefficients agree, both for $A=Y$ and $A=\nabla^{2}$, so we get extremal results. But just as in dimension two, the $Y$ signs disagree with the $\nabla^{2}$ signs, so we have opposite kinds of extrema. This is summarized in the first two lines of Table 0.1 .

As is clear from the third line, the sign miracles continue in dimension six (where in fact they seem more improbable, in that more coefficients have to fall into place with the correct sign). Note that the difference between the behavior 
TABLE 0.1. The functional determinant at the standard metric and its conformal transforms

\begin{tabular}{|c|c|c|}
\hline & $\operatorname{det} Y_{\omega}$ & $\operatorname{det} \nabla_{\omega}^{2}$ \\
\hline$S^{2}$ & $\max$ & $\min$ \\
\hline$S^{4}$ & $\min$ & $\max$ \\
\hline$S^{6}$ & $\max$ & $\min$ \\
\hline
\end{tabular}

of $Y$ and $\not \nabla^{2}$ is essential and is not due to conventions; we use the same convention in each case (i.e., that the leading symbol is multiplication by $|\xi|^{2}$ ). The third line of the table is the main new "factual" result of the present work:

Theorem 0.1. On $S^{6}$, for $g=g_{\omega}=e^{2 \omega} g_{0}$ in the conformal class of the standard metric $g_{0}$ and having the standard volume $16 \pi^{3} / 15$, the quantity det $Y_{\omega}$ (resp. $\operatorname{det} \not_{\omega}^{2}$ ) is maximized (resp. minimized) exactly when $g_{\omega}=h^{*} g_{0}$ for some conformal diffeomorphism $h$ on $\left(S^{6}, g_{0}\right)$.

The use of a generalized Polyakov formula to compute quotients of the form (0.1) requires a fairly explicit knowledge of one of the heat kernel invariants of the operator $A$ in question, that at the "index level"; i.e., that with a homogeneity degree (under uniform dilation of the metric) which compensates that of the Riemannian measure. We call this invariant $U_{m}[A]$. It is often said that the combinatorial complexity of the heat invariants explodes as one goes up in level; since level equals dimension in our problem, our difficulties explode as the dimension goes up. It has been possible, and hopefully not completely tasteless, to compute in dimension 6 by paying close attention to the dependence of all quantities on dimension; that is, by performing what physicists might call dimensional regularization. Specifically, we deal with universal polynomial formulas whose coefficients are rational functions of the dimension $m$, and use computations valid for large even $m$ to make conclusions about a specific $m$. In other words, we meromorphically continue rational functions of $m$, using $m=\infty$ as the limit point. This point of view excuses us from considering pure divergence terms in the heat invariant $U_{m}[A]$ in some subcomputations. The advantage gained from this is nonexistent in dimension 2 , minor in dimension 4 , and considerable in dimension 6 . To make things even more tractable, we work in the conformally flat category; this is of course stable under the changes of metric we want to consider, and does not discard the case of the sphere.

Extrapolating from dimensions 2, 4, and 6, the philosophy would seem to be as follows: the term corresponding to the exponential class Beckner-MoserTrudinger inequality [Bec], i.e. that related to the norm computation for the embedding $L_{m / 2}^{2} \hookrightarrow e^{L}$, is, in a sense to be made precise, the leading term in the functional determinant. Its coefficient is related to the leading term (really an equivalence class in the space of metric invariants) of $U_{m}[A]$; such leading terms were studied in [G5, BGØ1]. Looking at these formulas, one finds that if the leading term alone were decisive, the result would be the obvious 
continuation of Table 0.1 to arbitrary even $m$. The sign miracles, then, consist in coeffients of "trailing terms" having the correct sign. The estimation of the trailing terms, by the time we get to dimension 6, entails some relatively delicate conformal geometry. To oversimplify quite a bit, what ends up being used, in addition to the embedding $L_{3}^{2} \hookrightarrow e^{L}$, is the borderline embedding $L_{1}^{2} \hookrightarrow$ $L^{3}$, both in the trivial scalar bundle, and in the bundle TFS $^{2}$ of trace free symmetric two-tensors. More specifically, we have to study the effect of the scalar embedding on the scalar curvature function, and of the TFS $^{2}$ embedding on the Einstein (trace free Ricci) tensor.

Our treatment depends heavily on two pieces of technology that have only recently come clearly into view. The first is the construction by Graham, Jenne, Mason, and Sparling [GJMS] of conformally covariant operators on scalar functions with leading term $\Delta^{n / 2}, n$ a positive even integer, as long as it is not the case that the dimension $m$ is even and less than $n$. In particular, this allows us to find a representative of the leading term equivalence class described above which behaves relatively simply under conformal variation. The other is Beckner's recent work on sharp Sobolev and Moser-Trudinger inequalities, based on fundamental work of Lieb [Lie] on best constants and extremals for HardyLittlewood-Sobolev inequalities in $\mathbb{R}^{m}$. In Section 3 below, we give proofs of some of Beckner's results that use group invariance (and indeed, the complementary series) in an essential way.

The sequence of events in this paper will be as follows. Section 1 is about conformal covariance in general, and the Graham-Jenne-Mason-Sparling operators $P_{n}$ in particular. Something of extreme importance to us is the zeroth order term $Q_{n}$ of such a $P_{n}$, defined here by applying $P_{n}$ to the constant function: $P_{n} 1=(m-n) Q_{n} / 2$, where $m$ is the dimension. Section 2 is about the principal series of $\mathrm{SO}_{0}(m+1,1)$, and especially its subseries, the complementary series. Here we show how to compute the spectrum of an intertwining operator, and relate such operators to the operators $P_{n}$ of the previous section. Section 3 presents Beckner's theory of sharp inequalities on the sphere (i.e., that part of the theory that we shall need, from a point of view that suits our needs), with heavy emphasis on connections to the complementary series. Section 4 presents joint work of Branson and Ørsted on conformal index theory and the generalized Polyakov formula. Section 5 presents the two- and four-dimensional theory from the dimensional regularization point of view; the end results are somewhat different derivations of known results of Onofri and of Branson and Ørsted. In Section 6, we do the computations necessary to get formulas for $\operatorname{det} Y$ and $\operatorname{det} \not^{2}$ in six dimensions. In Section 7, these are applied to prove Theorem 0.1. A large part of Section 7 is devoted to some delicate conformal geometry and a new sharp inequality that seems to be necessary to the proof. Section 8 contains a discussion of the sign pattern of Table 0.1 and its continuation, and of prospects for future work.

It is a pleasure to thank Bill Beckner, Alice Chang, Peter Gilkey, Bent Ørsted, and Paul Yang for their help in clarifying the thinking of this paper. This material was part of a series of talks given by the author at the First Global Analysis Research Center Symposium on Pure and Applied Mathematics at Seoul National University; thanks are due to the organizers for making the talks possible. Finally, the author thanks Roskilde University Center, Seoul National 
University, and Sonderforschungsbereich 170 at the University of Göttingen for their hospitality, and the Danish Research Council for financial support, during the writing of this paper.

\section{CONFORMALLY COVARIANT DIFFERENTIAL OPERATORS ON SCALAR FUNCTIONS}

Let $(M, g)$ be a smooth, compact Riemannian manifold without boundary. $m$ will always denote the dimension of $M$. Let $\nabla$ be the Levi-Civita connection and $R$ be the Riemann curvature tensor of $g$. We shall say that a differential operator $D$ on smooth scalar functions on $M$ is natural if it can be given by a universal polynomial expression in $g$, its inverse $g^{\sharp}, \nabla$, and $R$, using tensor product and contraction. Such an object, of course, is not really an operator, but a rule that canonically assigns an operator to each Riemannian manifold of a given dimension. For example, $D$ could be the ordinary Laplacian $\Delta=\delta d$, where $d$ is the exterior derivative and $\delta$ its formal adjoint, since in the usual invariant index notation, $\Delta=-g^{i j} \nabla_{i} \nabla_{j}$. A local scalar invariant can be described as a natural differential operator of order zero acting on scalar functions; or alternatively, as a linear combination of monomials

$$
\mathscr{C}\left(\left(\nabla_{a_{1}} \ldots \nabla_{a_{p}} R_{i j k l}\right) \ldots\left(\nabla_{b_{1}} \ldots \nabla_{b_{q}} R_{\text {stuv }}\right)\right),
$$

where $\mathscr{C}$ is some operator which groups indices into pairs, raises one index in each pair, and contracts to a scalar.

We shall also have some use for natural operators between bundles of tensorspinors. Recall that the structure group of Riemannian geometry is $\mathrm{O}(m)$; and that of oriented Riemannian spin geometry is Spin $(m)$. (By Weyl's invariant theory, (1.1) is a basis of the local scalar $\mathrm{O}(m)$ invariants; see, e.g., [BFG, Section 5.8].) If $H$ is one of these structure groups, a tensor-spinor bundle is just a vector bundle of the form $\mathscr{F}_{H} \times_{\lambda} V$, where $(V, \lambda)$ is a finite-dimensional representation of $H$, and $\mathscr{F}_{H}$ is the bundle of $H$-frames. To define a natural operator between two such bundles, we just impose the polynomial restriction above, noting that if $H=\operatorname{Spin}(m)$, the Levi-Civita connection is extended to the Levi-Civita spin connection (which we also call $\nabla$ ), and the Clifford section $\gamma$ is allowed to enter our polynomial constructions. $\gamma$ is a section of $T M \otimes \operatorname{End}(\Sigma M)$, where $\Sigma M$ is the spinor bundle. For example, the Dirac operator $\not=\gamma^{i} \nabla_{i}$ is a natural $\operatorname{Spin}(m)$-operator on sections of $\Sigma M$. (The orientation assumed in using the structure group $\operatorname{Spin}(m)$ is just a convenience, and we shall not consider operators that depend on orientation.)

A natural differential operator $D$ is conformally covariant of bidegree $(a, b)$ $\in \mathbb{R}^{2}$ in dimension $m$ if for any $m$-dimensional Riemannian manifold $\left(M, g_{0}\right)$,

$$
g_{\omega}=e^{2 \omega} g_{0}, \omega \in C^{\infty}(M) \Rightarrow D_{\omega}=e^{-b \omega} D_{0} \mu\left(e^{a \omega}\right),
$$

where for any $f \in C^{\infty}(M), \mu(f)$ is multiplication by $f$. Here the subscripts indicate the metric in which we are evaluating; for example, $D_{0}$ is computed in $g_{0}$, and $D_{\omega}$ in $g_{\omega}$. We fix this subscript notation for use whenever we vary the metric within a conformal class

$$
\left[g_{0}\right]:=\left\{g_{\omega}=e^{2 \omega} g_{0} \mid \omega \in C^{\infty}(M)\right\} .
$$

We shall also apply the subscript notation to other quantities, for example, local invariants and the Riemannian measure $d v$. If $\operatorname{Spin}(m)$ bundles are 
involved in the construction of the operator $D$, we impose the compatible scaling $\gamma_{\omega}=e^{-\omega} \gamma_{0}$ in (1.2); the weight (coefficient of $\omega$ in the exponential) used in this scaling is forced by the Clifford relations.

We shall sometimes have use for the infinitesimal form of the conformal covariance relation (1.2). Suppose $\omega \in C^{\infty}(M)$, and consider the curve $g_{\varepsilon \omega}$ of conformal metrics; then (1.2) implies that

$$
\left.(d / d \varepsilon)\right|_{\varepsilon=0} D_{\varepsilon \omega}=-(b-a) \omega D_{0}+a\left[D_{0}, \mu(\omega)\right] .
$$

where $[\cdot, \cdot]$ is the commutator. All such variational formulas will be meant in the elementary sense: if $\varphi$ is a smooth section and $x \in M$, then

$$
\left(\left(\left.(d / d \varepsilon)\right|_{\varepsilon=0} D_{\varepsilon \omega}\right) \varphi\right)(x):=\left.(d / d \varepsilon)\right|_{\varepsilon=0}\left(\left(D_{\varepsilon \omega} \varphi\right)(x)\right) .
$$

The finite and infinitesimal forms of the conformal covariance relation are, in fact, equivalent: an application of (1.3) with $g_{\varepsilon_{0} \omega}$ in place of $g_{0}$ gives

$$
\left.(d / d \varepsilon)\right|_{\varepsilon=\varepsilon_{0}}\left\{e^{b \varepsilon \omega} D_{\varepsilon \omega} \mu\left(e^{-a \varepsilon \omega}\right)\right\}=0
$$

for any $\varepsilon_{0} \in \mathbb{R}$.

The best-known example of a conformal covariant is the conformal Laplacian, or Yamabe operator

$$
Y=\Delta+\frac{m-2}{4(m-1)} \tau, \quad m \neq 1,
$$

where $\tau$ is the scalar curvature $R^{i j}{ }_{i j}$ of $g$. (Our convention on placement of indices will be the one that makes $R^{1}{ }_{212}$ positive on standard spheres.) The Yamabe operator has conformal bidegree $((m-2) / 2,(m+2) / 2)$. Somewhat less well-known is the fourth-order Paneitz operator $P_{4}$, which was introduced in [P] (see also [Bra1, Theorem 1.21], and [ES]). To describe $P$, let $\rho$ be the Ricci tensor of $\nabla ; \rho_{i j}=R^{k}{ }_{i k j}$, and let

$$
\begin{aligned}
& J=\tau /(2(m-1)), \quad m \neq 1, \\
& V=(\rho-J g) /(m-2), \quad m \neq 1,2 .
\end{aligned}
$$

Then

$$
P=\Delta^{2}+\delta T d+(m-4) Q / 2, \quad m \neq 1,2,
$$

where

$$
\begin{aligned}
& T=(m-2) J-4 V \cdot, \\
& Q=\frac{m}{2} J^{2}-2|V|^{2}+\Delta J
\end{aligned}
$$

Here $V$. is the natural action of a two-tensor on one-forms, $(V \cdot \varphi)_{j}=V^{i}{ }_{j} \varphi_{i}$, and $|V|^{2}=V^{i j} V_{i j}$. The conformal bidegree of $P$ is $((m-4) / 2,(m+4) / 2)$.

The examples of $Y$ and $P$ make it clear that the dependence of a conformal covariant on the dimension $m$ is potentially important. Indeed, this dependence can be used to derive the Gauss curvature prescription equation in dimension 2 from the Yamabe equation in higher dimension. Applying the conformal covariance relation for $Y=\Delta+(m-2) J / 2$ to the constant function 1 , we get

$$
\left(\Delta+\frac{m-2}{2} J\right)_{0} e^{\frac{m-2}{2} \omega}=\frac{m-2}{2} J_{\omega} e^{\frac{m+2}{2} \omega}, \quad \omega \in C^{\infty}(M) .
$$


Since $\Delta$ annihilates constants,

$$
\Delta_{0}\left(e^{\frac{m-2}{2} \omega}-1\right)+\frac{m-2}{2} J_{0} e^{\frac{m-2}{2} \omega}=\frac{m-2}{2} J_{\omega} e^{\frac{m+2}{2} \omega} .
$$

Because the coefficients appearing in the expressions of $Y$ and $J$ as formal polynomials are rational in $m$, as are all terms in the conformal covariance relation for $Y$ (after multiplication by appropriate exponential factors), we may apply analytic continuation; specifically, we can divide by $(m-2) / 2$ and then evaluate at $m=2$ to get

$$
\Delta_{0} \omega+J_{0}=J_{\omega} e^{2 \omega}, \quad m=2 .
$$

Thus the Yamabe equation, with its power function nonlinearity (viewed as an equation on $\left.e^{(m-2) \omega / 2}\right)$, goes over to an equation with an exponential nonlinearity in $\omega$. On the level of identities, this reflects the transition on the level of inequalities from the borderline Sobolev embedding $L_{1}^{2} \hookrightarrow L^{2 m /(m-2)}$ to the Moser-Trudinger inequality as $m \downarrow 2$ (see Section 3 below). Clearly the same argument describes the behavior of the Paneitz operator $P$ and Paneitz quantity $Q$ as we approach the critical dimension, in this case $m=4$ : starting with the identity

$$
\left(P^{0}+\frac{m-4}{2} Q\right)_{0} e^{\frac{m-4}{2} \omega}=\frac{m-4}{2} Q_{\omega} e^{\frac{m+4}{2} \omega},
$$

where $P^{0}=P-(m-4) Q / 2$, we get

$$
P_{0} \omega+Q_{0}=Q_{\omega} e^{4 \omega}, \quad m=4,
$$

since $P^{0}$ annihilates constants, and $P=P^{0}$ in dimension 4 . The corresponding event on the level of inequalities is the transition from the borderline Sobolev embedding $L_{2}^{2} \hookrightarrow L^{2 m /(m-4)}$ to an exponential class inequality as $m \downarrow 4$; such inequalities have been studied in different settings by Lieb [Lie], Adams [A], and Beckner [Bec].

Onofri [On] and Osgood, Phillips, and Sarnak [OPS1-2] showed that the Moser-Trudinger inequality is decisive in the study of the functional determinant of the Laplacian as a functional on the space of metrics in dimension 2 . Branson, Chang, and Yang [BCY] showed that the exponential class inequality mentioned just above, together with the "conventional" borderline inequality $L_{1}^{2} \hookrightarrow L^{4}$, play a similar role in dimension 4 . Here the Paneitz operator, which arises naturally in four-dimensional determinant computations of Branson and Ørsted [BØ3], plays a central role. This is one of several motivations for a study of higher-order generalizations of $Y$ and $P$. Graham, Jenne, Mason, and Sparling [GJMS] have recently made such a study; we collect and strengthen some of their results here in a form that will be useful to us.

Theorem 1.1. Let $n$ be a positive even integer. Suppose that

$$
m \text { is odd, or } n \leq m \text {. }
$$

There is a conformally covariant differential operator $P_{n}$ on scalar functions, of conformal bidegree $((m-n) / 2,(m+n) / 2)$, such that:

(a) The leading symbol of $P_{n}$ is that of $\Delta^{n / 2}$. On $\mathbb{R}^{m}$ with its standard metric, $P_{n}=\Delta^{n / 2}$. 
(b) $P_{n}=P_{n}^{0}+\frac{m-n}{2} Q_{n}$, where $Q_{n}$ is a local scalar invariant and $P_{n}^{0}$ has the form $S_{n-1} d$ for some natural differential operator $S_{n-1}$ carrying one-forms to functions; in particular, $P_{n}^{0}$ annihilates constants.

(c) There are universal expressions for $P_{n}$ and $Q_{n}$ as polynomials in $\left(g, g^{\sharp}\right.$, $R, \nabla)$ with coefficients that are real rational functions of $m$, regular at all positive integral $m$ satisfying (1.6).

Proof. The assertions on the existence of $P_{n}$ and its conformal bidegree, together with part (a), are contained in [GJMS].

For parts (b) and (c), we examine the construction of $P_{n}$ given in [GJMS]. This construction is local, and involves a manifold $\tilde{G}$ of dimension $m+2$. $\tilde{G}$ is covered by charts $U \times \mathbb{R} \times(0,1)$, where the $U$ are charts for a given Riemannian manifold $(M, g)$; the coordinates on $U$ (resp. the $\mathbb{R}$ and $(0,1)$ factors), will be called $x=\left(x^{1}, \ldots, x^{m}\right)$ (resp. $u$ and $s$ ). In [GJMS], what we call $e^{u}$ is called $t$, and what we call $s$ is called $\rho . \tilde{G}$ is endowed with a metric

$$
\tilde{g}=e^{2 u}\left\{g_{i j}(x, s) d x^{i} d x^{j}+2 s d u^{2}+2 d u d s\right\}
$$

of signature $(m+1,1)$ which is defined formally by the requirement that the Ricci tensor $\tilde{\rho}$ of $\tilde{g}$ vanishes. That is, one computes a Taylor series in $s$ for $\tilde{g}$ based on this requirement, together with the initial condition $g_{i j}(x, 0)=$ $g_{i j}(x)$. The result is the equation

$$
s\left(g_{i j}^{\prime \prime}-g^{k l} g_{i k}^{\prime} g_{j l}^{\prime}+\frac{1}{2} g^{k l} g_{k l}^{\prime} g_{i j}^{\prime}\right)+\frac{2-m}{2} g_{i j}^{\prime}-\frac{1}{2} g^{k l} g_{k l}^{\prime} g_{i j}+\rho_{i j}=0 .
$$

(See [GJMS, equation (3.2)].) Here the prime is $s$-differentiation, and $\rho$ is the Ricci tensor of the metric $g_{i j}(x, s)$ for fixed $s$. An inductive construction of the Taylor series is possible if $m$ is odd; when $m$ is even, the series is determined only up to order $s^{m / 2}$. The operator $P_{n}$ arises as the obstruction to finding an extension, near the $s=0$ hypersurface, of a function $e^{(n-m) u / 2} \varphi(x)$ to a function $e^{(n-m) u / 2} \varphi(x, s)$ which is annihilated by the Laplacian $\square$ of the metric $\tilde{g}$. That is, the obstruction to extending $e^{(n-m) u / 2} \varphi(x)$ is $P_{n} \varphi$. This obstruction is obtained by solving for a Taylor expansion of $\varphi(x, s)$ using the equation

$$
-2 s \varphi^{\prime \prime}+\left(n-2-s g^{i j} g_{i j}^{\prime}\right) \varphi^{\prime}-\Delta \varphi+\frac{1}{4}(n-m) g^{i j} g_{i j}^{\prime} \varphi=0 .
$$

Here $\Delta$ is the Laplacian of $g_{i j}(x, s)$ for fixed $s$. (Compare [GJMS, (3.5)], noting that the reference's $n$ is our $m$, the reference's $-\Delta$ is our $\Delta$, and the reference's $w$ is $(n-m) / 2$ in the case under consideration.) The obstruction appears when one takes $\frac{n}{2}-1$ derivatives of (1.7) with respect to $s$; it appears in the form $K(m, n) P_{n} \varphi$, where $K$ is rational in $m$ for fixed $n$. (Here we are taking care to see that the normalization $P_{n}=\Delta^{n / 2}+$ (lower order) preserves rationality. $K(m, n)$ is the $C_{n, w, l}$ of [GJMS, p. 564].) Since the entire construction is rational in $m$, the coefficients of $P_{n}$ are also rational in $m$.

When $n=m$, the local invariant $P_{n} 1$ is the obstruction to extending the constant function 1 to a function on $G$ annihilated by $\square$ (i.e., we try to extend $e^{(n-m) u / 2} \cdot 1$ with $\left.n=m\right)$. Since $\square 1=0$, there is no such obstruction; the desired extension is 1 . Thus $P_{m} 1=0$. But

$$
P_{n}=P_{n}^{0}+\tilde{Q}_{n},
$$


where $\tilde{Q}_{n}$ is the local invariant $P_{n} 1$, and $P_{n}^{0}$ has the form $\left(S_{n-1}\right)^{i} \nabla_{i}=S_{n-1} d$. Since $\tilde{Q}_{m}=0$, we can write $\tilde{Q}_{n}=(m-n) Q_{n} / 2$; further, all coefficients are still rational in $m$. This completes the proof of (b) and (c).

The theorem does not assert uniqueness of the $P_{n}$. In fact, it is easy to show nonuniqueness if $n$ is a multiple of $4:$ if $C$ is the Weyl conformal curvature tensor,

$$
C^{i}{ }_{j k l}=R^{i}{ }_{j k l}+V_{j k} \delta^{i}{ }_{l}-V_{j l} \delta^{i}{ }_{k}+V^{i}{ }_{l} g_{j k}-V^{i}{ }_{k} g_{j l},
$$

scalar multiples of $|C|^{n / 2}=\left(C^{i j k l} C_{i j k l}\right)^{n / 4}$ can be added to $P_{n}$ without disturbing conformal covariance. In the next section, however, we shall show that the evaluation of $P_{n}$ on the sphere $S^{m}$ with its standard metric $g_{0}$, or with any conformal multiple of $g_{0}$, is unique.

Note that rationality in $m$ is preserved upon changes of basis resulting when the generators $R, \rho, \tau$ are replaced by $C, V, J$, and vice versa; we shall often use this fact implicitly.

The operators $Y$ and $P$ fill the requirements set by the theorem for $P_{2}$ and $P_{4}$, with $J$ and $Q$ as the corresponding $Q_{2}$ and $Q_{4}$. In fact, some elementary invariant theory shows that $P_{2}$ is uniquely determined to be $Y$, and $P_{4}$ must have the form $P+\alpha(m)|C|^{2}$ for some rational function $\alpha(m)$.

Remark 1.2. There is no formal difference in the above discussion between Riemannian and pseudo-Riemannian conformal geometry: the formation of monomials $(1.1)$ is the same, and the finite dimensional representation theory of the structure group $\operatorname{Spin}_{0}(p, q), p+q=m$, is the same as that of $\operatorname{Spin}_{0}(m)$ by the Weyl unitary trick. In particular, the operators of Theorem 1.1 exist in the pseudo-Riemannian regime, and are given by the same formal polynomials as in the Riemannian case.

Remark 1.3. It does not seem to be apparent from the [GJMS] construction that the $P_{n}$ are formally self-adjoint, though this is a natural conjecture. If $D$ is a natural scalar differential operator of conformal bidegree $(a, b)$, an easy argument shows that $D^{*}$ has conformal bidegree $(m-b, m-a)$. Thus $P_{n}^{*}$ satisfies the requirements set by the theorem for $P_{n}$, except for part (b): $P_{m}^{*} 1$ might not vanish. Thus we cannot just switch consideration to the selfadjoint part $\left(P_{n}+P_{n}^{*}\right) / 2$ without investigating the [GJMS] construction further. Instead, we content ourselves with the following observation, which is good enough for our purposes: If $\left[g_{0}\right]$ is a given conformal class on a Riemannian manifold $M$, and $P_{n}$ is formally self-adjoint at $g_{0}$, then $P_{n}$ is formally selfadjoint at all metrics $g_{\omega} \in\left[g_{0}\right]$. Indeed, if $\left(P_{n}\right)_{0}$ is formally self-adjoint in the metric $g_{0}$ and $\varphi$ or $\psi$ has compact support,

$$
\begin{aligned}
\int_{M}\left\{\left(P_{n}\right)_{\omega} \varphi\right\} \psi(d v)_{\omega} & =\int_{M} e^{-\frac{m+n}{2} \omega}\left\{\left(P_{n}\right)_{0}\left(e^{\frac{m-n}{2} \omega} \varphi\right)\right\} \psi e^{m \omega}(d v)_{0} \\
& =\int_{M}\left\{\left(P_{n}\right)_{0}\left(e^{\frac{m-n}{2} \omega} \varphi\right)\right\}\left(e^{\frac{m-n}{2} \omega} \psi\right)(d v)_{0} \\
& =\int_{M}\left(e^{\frac{m-n}{2} \omega} \varphi\right)\left\{\left(P_{n}\right)_{0}\left(e^{\frac{m-n}{2} \omega} \psi\right)\right\}(d v)_{0}
\end{aligned}
$$

showing that the first expression is symmetric in $\varphi$ and $\psi$, and thus that $\left(P_{n}\right)_{\omega}$ is formally self-adjoint in the metric $g_{\omega}$. As a special case of this observation, 
we get that $P_{n}$ is formally self-adjoint in any conformally flat metric, since $P_{n}=\Delta^{n / 2}$ on standard $\mathbb{R}^{m}$.

Although it is not our intention to try to write an explicit formula for $Q_{n}$ except in low dimension, its conformal behavior can be described in arbitrary dimension:

Corollary 1.4. If $(m, n)$ is as in (1.6) and $P_{n}$ is formally self-adjoint at $g_{0}$, then

$$
\left.(d / d \varepsilon)\right|_{\varepsilon=0} \int\left(Q_{n} d v\right)_{\varepsilon \omega}=(m-n) \int \omega\left(Q_{n} d v\right)_{0} .
$$

If $m$ is even and $P_{m}$ is formally self-adjoint at $g_{0}$, then

$$
\int\left(Q_{m} d v\right)_{\omega}=\int\left(Q_{m} d v\right)_{0}
$$

Proof. The conformal covariance relation for $P_{n}$, applied to the constant function 1 , reads

$$
\left(P_{n}^{0}+\frac{m-n}{2} Q_{n}\right)_{0} e^{\frac{m-n}{2} \omega}=\frac{m-n}{2}\left(Q_{n}\right)_{\omega} e^{\frac{m+n}{2} \omega} .
$$

Just as for the cases $n=2,4$, we can analytically continue to compute the conformal behavior of $Q_{m}$, which is not explicitly given by (1.11). Since $P_{n}^{0}=$ $S_{n-1} d$ annihilates constants,

$$
\left(P_{n}^{0}\right)_{0}\left(e^{\beta \omega}-1\right)+\beta\left(Q_{n}\right)_{0} e^{\beta \omega}=\beta\left(Q_{n}\right)_{\omega} e^{(m-\beta) \omega}, \quad \beta=(m-n) / 2 .
$$

By Theorem 1.1(c), we can divide by $\beta$ and evaluate at $\beta=0$ :

$$
\left(P_{m}\right)_{0} \omega+\left(Q_{m}\right)_{0}=\left(Q_{m}\right)_{\omega} e^{m \omega}, \quad m \text { even. }
$$

Since $(d v)_{\omega}=e^{m \omega}(d v)_{0}$,

$$
\left(\left(P_{m}\right)_{0} \omega+\left(Q_{m}\right)_{0}\right)(d v)_{0}=\left(Q_{m} d v\right)_{\omega}, \quad m \text { even } .
$$

The infinitesimal form of (1.11) is

$$
\left.(d / d \varepsilon)\right|_{\varepsilon=0}\left(Q_{n}\right)_{\varepsilon \omega}+n \omega\left(Q_{n}\right)_{0}=\left(P_{n}^{0}\right)_{0} \omega,
$$

in all dimensions $m$ described by (1.6), except $m=n$. By analytic continuation, this extends also to $m=n$. So far we have not used the formal self-adjointness assumption.

For a conformal class with $P_{m}$ formally self-adjoint (Remark 1.3), $P_{m}=$ $\delta S_{m-1}^{*}$, so (1.13) and Stokes' Theorem give (1.10). Since $\left.(d / d \varepsilon)\right|_{\varepsilon=0}(d v)_{\varepsilon \omega}=$ $m \omega(d v)_{0}$, the same sort of reasoning leads from (1.15) to (1.9).

It is also possible to say something about the terms in $Q_{n}$ with the most and least derivatives. To make this precise, fix $m$ and consider the space $\mathscr{J}_{n}$ of local scalar $\mathrm{O}(m)$ invariants $A$ which have level $n$ in the sense that a uniform scaling $\bar{g}=\alpha^{2} g, 0<\alpha \in \mathbb{R}$, results in $\bar{A}=\alpha^{-n} A$. It is easy to see that the monomial (1.1) is in $\mathscr{I}_{n}$ with

$$
n=(2+p)+\ldots+(2+q) \text {. }
$$

There is a natural vector space filtration

$$
\mathscr{I}_{n, 0} \subset \mathscr{I}_{n, 2} \subset \ldots \subset \mathscr{I}_{n, n-2}=\mathscr{I}_{n}
$$


of $\mathscr{I}_{n}$ defined by declaring (1.1) to be in $\mathscr{I}_{n, N}$ if $(1.16)$ holds and $p+\ldots+q \leq$ $N$. (Of course, applications of the curvature identities to a quantity which appears only to be in $\mathscr{I}_{n, N}$ may show that that quantity is actually in the more elite space $\mathscr{I}_{n}, N^{\prime}, N^{\prime}<N$.) For example, the $m$-dimensional Pfaffian $\mathrm{Pff}_{m}$ is an element of $\mathscr{I}_{m, 0}$. The terms in $Q_{n}$ with the most derivatives are described by the following.

Corollary 1.5. If $Q_{n}$ is as in Theorem 1.1, then $Q_{n} \simeq \Delta^{(n-2) / 2} J$ modulo $\mathscr{I}_{n, n-4}$. Proof. Fix $m$ and consider the following conformal variation operator $\operatorname{Var}_{n}$ on $\mathscr{I}_{n}$ :

$$
\operatorname{Var}_{n}(\omega, A)=\left.(d / d \varepsilon)\right|_{\varepsilon=0} A_{\varepsilon \omega}+n \omega A_{0}, \quad \omega \in C^{\infty}(M) .
$$

An inductive argument [Bra1, Section 1.b] shows that the target space for $\operatorname{Var}_{n}$ is a space $\mathscr{J}_{n}$ of $d \omega$-augmented local invariants generated by formal monomials

$$
\mathscr{C}\left(\left(\nabla_{a_{1}} \ldots \nabla_{a_{p}} R_{i j k l}\right) \ldots\left(\nabla_{b_{1}} \ldots \nabla_{b_{q}} R_{\text {stuv }}\right)\left(\nabla_{c_{1}} \ldots \nabla_{c_{r}} \omega\right)\right)
$$

where $\mathscr{C}$ is as described after (1.1), $r>0$, and $(2+p)+\ldots+(2+q)+r=n$. Here $\omega$ is to be viewed as an indeterminate element of $C^{\infty}(M)$. Just as for the unaugmented invariants above, the number $p+q+r$ of explicit derivatives defines a filtration

$$
\mathscr{J}_{n, 2} \subset \mathscr{J}_{n, 4} \subset \ldots \subset \mathscr{J}_{n, n}=\mathscr{J}_{n}
$$

and it is easily shown that

$$
\operatorname{Var}_{n}\left(\cdot, \mathscr{I}_{n, N}\right) \subset \mathscr{J}_{n, N+2} .
$$

(Again, see [Bra1, Section 1.b].) Elementary invariant theory [BGØ2, Section 1] shows that $\mathscr{I}_{n} / \mathscr{I}_{n, n-4}$ and $\mathscr{I}_{n} / \mathscr{I}_{n, n-2}$ are one-dimensional, with generators given by the equivalence classes of $\Delta^{(n-2) / 2} J$ and $\Delta^{n / 2} \omega$ respectively. In particular, there is a constant $a$ (which may a priori depend on $m$ and $n$ ) for which

$$
Q_{n}-a \Delta^{(n-2) / 2} J \in \mathscr{I}_{n, n-4} .
$$

The conformal covariance relation for $Y$ and its consequence (1.5) show that

$$
\operatorname{Var}_{n}\left(\omega, \Delta^{(n-2) / 2} J\right) \simeq \Delta^{n / 2} \omega \text { modulo } \mathscr{J}_{n, n-2}
$$

By (1.15),

$$
\operatorname{Var}_{n}\left(\omega, Q_{n}\right)=P_{n}^{0} \omega \simeq \Delta^{n / 2} \omega \text { modulo } \mathscr{J}_{n, n-2}
$$

in all dimensions $m$ satisfying (1.6). This and (1.19) identify the constant $a$ in (1.18) as 1 , completing the proof.

We can also write down the term in $\int Q_{n} d v$ with the most derivatives. Note that exact divergences, i.e., local scalar invariants in the range of the formal adjoint $\delta$ of the exterior derivative $d$, integrate to zero, and that it is precisely the exact divergences that integrate to zero universally.

Corollary 1.6. Suppose $n>4$. In the conformally flat case, if $Q_{n}$ is as in Theorem 1.1, then $Q_{n} \simeq \Delta^{(n-2) / 2} J+\frac{m-n}{2}\left|\nabla^{(n-4) / 2} J\right|^{2}$ modulo $\mathscr{I}_{n, n-6}+(\mathscr{R}(\delta) \cap$ $\mathscr{I}_{n, n-4)}$.

Proof. Invariant theory, Corollary 1.5, the Bianchi identity, and integration by parts show that $Q_{n} \simeq \Delta^{(n-2) / 2} J+b\left|\nabla^{(n-4) / 2} J\right|^{2}$, where $b$ is a constant depending 
on $m$ and $n$. But integrating by parts,

$$
\left.(d / d \varepsilon)\right|_{\varepsilon=0} \int\left(\left|\nabla^{(n-4) / 2} J\right|^{2} d v\right)_{\varepsilon \omega}=2 \int \omega\left(\left(\Delta^{(n-2) / 2} J+B\right) d v\right)_{0},
$$

where $B \in \mathscr{I}_{n, n-4}$. That $b=(m-n) / 2$ now follows from (1.17) and Corollary 1.4 .

Still more information about $Q_{n}$ can be gleaned from (1.11), and this information is crucial to an understanding of how a study of the functional determinant leads to the operators $P_{m}$. The problem that arises is to compute a conformal primitive, or integral, of a level $m$ local invariant $U$; this is a functional quantity $\mathscr{P}$ on the conformal class $\left[g_{0}\right]$ for which

$$
\left.(d / d \varepsilon)\right|_{\varepsilon=0} \mathscr{P}_{\omega+\varepsilon \eta}=\int \eta(U d v)_{\omega}, \quad \text { all } \omega, \eta \in C^{\infty}(M) .
$$

As usual, the convention is that a quantity subscripted with $\omega$ is evaluated in the metric $g_{\omega}$. Conformal primitives, when they exist, are unique up to constant functionals on $\left[g_{0}\right]$, since they solve first-order initial value problems in $\varepsilon$ when restricted to the curves $g_{\varepsilon \omega}$ for a fixed $\omega . Q_{m}$ enters because by Corollary 1.5 , it is the "leading term" of any such $U$ up to a constant factor. (Of course, $\Delta^{(m-2) / 2} J$ could also be used as the leading term, but the conformal behavior of $Q_{m}$ is much nicer.) In practice, the leading term is more difficult to estimate than "trailing terms"; an instance is the problem from [BCY] in dimension 4 mentioned above: the leading term corresponds to an exponential class embedding $L_{2}^{2} \hookrightarrow e^{L}$, while the (sole) trailing term is estimated by the ordinary borderline embedding $L_{1}^{2} \hookrightarrow L^{4}$. When we try to solve (1.20) with $U=Q_{n}$, all is straightforward for $m$ odd or $m>n$ : by (1.9), we may take $\mathscr{P}$ to be $\left(\int Q_{n} d v\right) /(m-n)$ if we are in a conformal class where $P_{n}$ is formally self-adjoint. When $m=n$, we get a solution $\mathscr{P}$ by analytic continuation: after division by $\beta$ in (1.12), we have

$$
\left\{\left(P_{n}^{0}\right)_{0}\left(\frac{e^{\beta \omega}-1}{\beta}\right)+\left(Q_{n}\right)_{0} e^{\beta \omega}\right\}(d v)_{0}=\left(Q_{n}\right)_{\omega} e^{-\beta \omega}(d v)_{\omega} .
$$

In the proof of Corollary 1.4, we just evaluated this at $m=n$; to get higherorder (in $\beta$ ) information, we rewrite (1.21) as

$$
\begin{aligned}
\left(Q_{n} d v\right)_{\omega}-\left(Q_{n} d v\right)_{0}=\left\{\left(P_{n}^{0}\right)_{0}\left(\frac{e^{\beta \omega}-1}{\beta}\right)+\left(e^{\beta \omega}-1\right)\left(P_{n}^{0}\right)_{0}\left(\frac{e^{\beta \omega}-1}{\beta}\right)\right. & \left.+\left(e^{2 \beta \omega}-1\right)\left(Q_{n}\right)_{0}\right\}(d v)_{0} .
\end{aligned}
$$

Now we inject the assumption that $\left(P_{m}\right)_{0}$ is formally self-adjoint, so that upon integration, we lose the first term on the right by Stokes' Theorem ( $P_{m}$ having the form $\left.\delta S_{m-1}^{*}\right)$. Dividing (1.22) by $2 \beta=m-n$, we have

$$
\begin{aligned}
& \int \frac{\left(Q_{n} d v\right)_{\omega}-\left(Q_{n} d v\right)_{0}}{m-n} \\
& \quad=\int\left\{\left(\frac{e^{\beta \omega}-1}{2 \beta}\right)\left(P_{n}^{0}\right)_{0}\left(\frac{e^{\beta \omega}-1}{2 \beta}\right)+\left(\frac{e^{2 \beta \omega}-1}{2 \beta}\right)\left(Q_{n}\right)_{0}\right\}(d v)_{0} .
\end{aligned}
$$


Evaluating at $m=n$, we get

$$
\left.\int \frac{\left(Q_{n} d v\right)_{\omega}-\left(Q_{n} d v\right)_{0}}{m-n}\right|_{m=n} "=" \frac{1}{2} \int \omega\left(P_{m}\right)_{0} \omega(d v)_{0}+\int \omega\left(Q_{m}\right)_{0}(d v)_{0} .
$$

We have not defined the class of meromorphic-in- $m$ local invariants appropriate to making this precise, but this will not be necessary. (The last analytic continuation was not strictly legal, since we imposed a restriction on the conformal class before performing it.) What we shall need is the following, the proof of which is immediate from (1.9) and (1.14):

Corollary 1.7. If $m$ is odd or $n<m$, and $P_{n}$ is formally self-adjoint at $g_{0}$, the functional

$$
\mathscr{P}_{(m, n)}: \omega \mapsto\left(\mathscr{P}_{(m, n)}\right)_{\omega}:=(m-n)^{-1} \int\left\{\left(Q_{n} d v\right)_{\omega}-\left(Q_{n} d v\right)_{0}\right\}
$$

is a conformal primitive for $Q_{n}$ in the sense of (1.20), and vanishes at $\omega=0$. If $m$ is even, and $P_{m}$ is formally self-adjoint at $g_{0}$, the functional

$$
\begin{aligned}
\left(\mathscr{P}_{(m, m)}\right)_{\omega} & =\frac{1}{2} \int \omega\left(\left(P_{m}\right)_{0} \omega\right)(d v)_{0}+\int \omega\left(Q_{m} d v\right)_{0} \\
& =\frac{1}{2} \int \omega\left\{\left(Q_{m} d v\right)_{\omega}+\left(Q_{m} d v\right)_{0}\right\}
\end{aligned}
$$

is a conformal primitive for $Q_{m}$ which vanishes at $\omega=0$.

\section{CONFORMAL COVARIANCE ON THE SPHERE, AND THE SPHERICAL PRINCIPAL SERIES OF $\mathrm{SO}_{0}(m+1,1)$}

Beckner [Bec] has pointed out that the meaning of Lieb's best constant for the Hardy-Littlewood-Sobolev inequality in $\mathbb{R}^{m}$ [Lie] is more apparent when this inequality is transferred to the setting of the sphere $S^{m}$ via stereographic projection. After a suggestion of Bent Ørsted, the present author pointed out that these constants, together with other constants which arise when the inequalities are written in terms of spherical harmonics, are actually data from the theory of spherical principal series representations of the semisimple Lie group $G=\mathrm{SO}_{0}(m+1,1)$ (see [Bec, Sections 2,4]). Here the word "spherical" just refers to the fact that these representations live in spaces of ordinary functions, as opposed to vector bundle sections. (Properly understood, they live in spaces of line bundle sections, but it is not necessary to view them in this light, and we intend to offer a totally elementary workout of the theory.)

The starting point is the realization of $G$ as (the identity component of) the conformal transformation group of the sphere with its standard metric $g$. In general, a conformal transformation on a Riemannian manifold $(M, g)$ is a diffeomorphism $h$ on $M$ with $h \cdot g=\Omega_{h}^{2} g$, where $0<\Omega_{h} \in C^{\infty}(M)$. Here $h$. is the natural pushout of tensors by a diffeomorphism; in particular, $h \cdot=\left(h^{-1}\right)^{*}$ on purely covariant tensors like $g$. The group of conformal transformations will be called $\operatorname{ctran}(M, g)$. The map $h \mapsto \Omega_{h}$ carrying $\operatorname{ctran}(M, g)$ to $C^{\infty}(M)_{+}$ is a multiplier, or cocycle:

$$
\Omega_{h_{1} \circ h_{2}}=\Omega_{h_{1}}\left(h_{1} \cdot \Omega_{h_{2}}\right) .
$$


The corresponding infinitesimal notion is that of conformal vector field. Let $\mathscr{D}^{1}(M)$ be the $C^{\infty}(M)$-module of smooth vector fields, and let $\mathscr{L}$ denote the Lie derivative. $X \in \mathscr{D}^{1}(M)$ is conformal if $\mathscr{L}_{X} g=2 \omega_{X} g$ for some $\omega_{X} \in$ $C^{\infty}(M)$; we denote the Lie algebra of conformal vector fields by $\operatorname{cvf}(M, g)$. The map $X \mapsto \omega_{X}$ carrying $\operatorname{cvf}(M, g)$ to $C^{\infty}(M)$ is an infinitesimal cocycle:

$$
\omega_{\left[X_{1}, X_{2}\right]}=X_{1} \omega_{X_{2}}-X_{2} \omega_{X_{1}}
$$

The connection between the finite and infinitesimal notion is made by integrating conformal vector fields to local one-parameter groups of local conformal transformations. The relation to conformal change of metric as treated in the last section is as follows: a conformal transformation $h$ can be thought of as a composition

$$
h:(M, g) \stackrel{h}{\longrightarrow}\left(M, \Omega_{h}^{2} g\right) \stackrel{\text { id }}{\longrightarrow}(M, g)
$$

of an isometry and a conformal change of metric. Thus a general conformal covariant $D$ of bidegree $(a, b)$ will have the behavior

$$
\begin{aligned}
D\left(\Omega_{h}^{a} h \cdot \varphi\right) & =\Omega_{h}^{b} h \cdot(D \varphi), \\
D\left(\mathscr{L}_{X}+a \omega_{X}\right) \varphi & =\left(\mathscr{L}_{X}+b \omega_{X}\right) D \varphi
\end{aligned}
$$

under the transformations above.

On the sphere, all conformal vector fields integrate globally. Indeed, the conformal action of $G$ on $S^{m}$ resolves the singularities in the action of the conformal group on $\mathbb{R}^{m}$ : under stereographic projection, the action is transferred to $S^{m}$, where the singular generator, inversion in the unit sphere, becomes reflection across an equator. To see the action concretely, we realize $S^{m}$ as the unit sphere $S_{1}^{m}$ in $\mathbb{R}^{m+1}$, with homogeneous coordinates $y=\left(y_{0}, \ldots, y_{m}\right)$, and also as $S_{1}^{m} \times\{1\} \subset \mathbb{R}^{m+2}$; the "extra" component will be called $y_{m+1}$. If $A \in G$, we can take the linear action of $A$ on $(y, 1)$ and then divide by $(A(y, 1))_{m+1}>0$ to get a new element $A \cdot y$ of $S_{1}^{m} \times\{1\}$. This action defines an isomorphism

$$
l: G \stackrel{\simeq}{\rightrightarrows} \operatorname{ctran}\left(S^{m}, g\right) .
$$

From the Lie-theoretic viewpoint, $G$ is the fundamental object, and the space $S^{m}$ arises as $G / P_{\min }$, where $P_{\min }$ is a minimal parabolic subgroup. As one does generally in semisimple theory, take a Cartan decomposition $\mathfrak{g}=\mathfrak{k}+\mathfrak{s}$ on the Lie algebra level, choose a maximal abelian Lie algebra $\mathfrak{a}$ in $\mathfrak{s}$, and fix a positive open Weyl chamber $\mathfrak{a}_{+}^{*}$ in $\mathfrak{a}^{*}$. Let $G=K A N$ be the corresponding Iwasawa decomposition, and let $M$ be the centralizer $\{m \in K \mid(\operatorname{Ad} m) H=H$, all $H \in$ a . The minimal parabolic subgroup corresponding to our choices is $P=$ $M A N$. The Iwasawa decomposition gives an identification of the homogeneous spaces $G / M A N$ and $K / M$.

In our case, and in the notation above, $K$ is the copy of $\mathrm{SO}(m+1)$ that acts in the $\left(y_{0}, \ldots, y_{m}\right)$ variables, and $M$ is the copy of $\mathrm{SO}(m)$ that acts in the $\left(y_{1}, \ldots, y_{m}\right)$ variables. Here and in the rest of this section, we assume that $m \geq 2$. The smooth action of $G$ infinitesimalizes to a Lie algebra isomorphism

$$
l: \mathfrak{g} \cong \operatorname{cvf}\left(S^{m}, g\right) .
$$

In homogeneous coordinates on $\mathbb{R}^{m+2}$, if $\varepsilon_{i}=1$ for $i=0, \ldots, m$ and $\varepsilon_{m+1}=$ $-1, l(\mathfrak{g})$ is spanned by the $L_{i j}=\varepsilon_{i} y_{i} \partial_{j}-\varepsilon_{j} y_{j} \partial_{i}$ for $0 \leq i, j \leq m+1$, and the 
commutation relations are

$$
\begin{aligned}
& {\left[L_{i j}, L_{j k}\right]=\varepsilon_{j} L_{i k},} \\
& {\left[L_{i j}, L_{k l}\right]=0 \text { if } i, j, k, l \text { are distinct. }}
\end{aligned}
$$

$l(\mathfrak{k})$ is spanned by the $L_{i j}$ with $0 \leq i, j \leq m$. By (2.5), a is one-dimensional; we make the choice $\mathfrak{a}=\mathbb{R} H_{0}$, where $l\left(H_{0}\right)=L_{0, m+1}$. As a result of this choice, $l(\mathfrak{m})=\operatorname{span}\left\{L_{i j} \mid 1 \leq i, j \leq m\right\}$. We also need to pick a positive Weyl chamber $\mathfrak{a}_{+}^{*}$; we do this by declaring the dual element $\alpha \in \mathfrak{a}^{*}$, i.e., the one with $\alpha\left(H_{0}\right)=1$, to be positive. To see the $(\mathfrak{g}, \mathfrak{a})$ root structure, note that $L_{i, m+1}+L_{0 i}$ (resp. $\left.L_{i, m+1}-L_{0 i}\right)$ is a +1 (resp. -1 ) eigenvector of $\left[L_{0, m+1}, \cdot\right]$ for $1 \leq i \leq m$. Thus there are two roots, $\pm \alpha$, with root spaces

$$
\mathfrak{g}_{ \pm \alpha}=l^{-1}\left(\operatorname{span}\left\{L_{i, m+1} \pm L_{0 i} \mid 1 \leq i \leq m\right\}\right) .
$$

The Iwasawa $\mathfrak{n}$ is the sum of the positive root spaces, here $\mathfrak{g}_{\alpha}$; its image $\theta \mathfrak{n}=\overline{\mathfrak{n}}$ under the Cartan involution $\theta$ is just $\mathfrak{g}_{-\alpha}$. The multiplicity of $\alpha$ is $\operatorname{dim} \mathfrak{g}_{\alpha}=m$, so the important quantity $\rho$, half the sum of the positive $(\mathfrak{g}, \mathfrak{a})$ roots, is $m \alpha / 2$.

A remark on normalizations is in order. From the Lie-theoretic point of view, natural Riemannian metrics on homogeneous spaces of $G$, when they exist, are determined by the Killing form $B_{\mathfrak{g}}(X, Y)=-\operatorname{tr}(\operatorname{ad} X$ ad $Y)$. The normalization

$$
b=b_{\mathfrak{s o}(p, q)}=B_{\mathfrak{s o}(p, q)} / 2(p+q-2)
$$

of Killing forms in the $\mathfrak{s o}(p, q)$ series has several convenient effects: (a) $b(X, Y)$ $=-\frac{1}{2} \operatorname{tr} \ell(X) \ell(Y)$ for $\ell$ the defining representation of $\mathfrak{s o}(p, q)$; (b) for any standard (block stabilization) inclusion $\mathfrak{s o}\left(p^{\prime}, q^{\prime}\right) \hookrightarrow \mathfrak{s o}(p, q)$, the restricted and intrinsic forms $b$ agree on the smaller algebra. If $\mathfrak{g}=\mathfrak{s o}(m+1,1)$ as above, we also get: (c) $|\alpha|=1$ in the corresponding inner product on $\mathfrak{g}^{*} ;$ (d) the manifold $K / M$ with the normalized Killing form metric is the standard $m$-sphere, which is distinguished by its scalar curvature $m(m-1)$. Indeed, by [Bes, 7.39], the scalar curvature of $K / M$ in the $B_{\mathfrak{k}}$ metric is $m / 2$. Our normalization divides the metric by $2(m-1)$, and thus multiplies the scalar curvature by $2(m-1)$, giving $m(m-1)$ as desired.

At this point, we are ready to set up the spherical principal series representations, at first in elementary differential geometric terms. It will be convenient to have at our disposal, in addition to $C^{\infty}\left(S^{m}\right)$, the smaller space $\mathscr{E}\left(S^{m}\right)$ of functions which are finite sums of spherical harmonics on $S^{m}$. Like $C^{\infty}\left(S^{m}\right)$ functions, $\mathscr{E}\left(S^{m}\right)$ is dense in any Sobolev space, and so its use will not cause problems later, when we close in Sobolev norms determined by the representation theory. In representation theoretic terms, $\mathscr{E}\left(S^{m}\right)$ is the space of $K$-finite vectors.

By the cocycle conditions $(2.1,2.2)$, the maps

$$
u_{\nu}(h)=\Omega_{h}^{\nu+\frac{m}{2}} h \cdot, \quad U_{\nu}(X)=X+\left(\nu+\frac{m}{2}\right) \omega_{X}
$$

for $\nu \in \mathbb{C}$, are homomorphisms

$$
\operatorname{ctran}\left(S^{m}, g\right) \stackrel{u_{\nu}}{\rightarrow} \text { Aut } C^{\infty}\left(S^{m}\right), \quad \operatorname{cvf}\left(S^{m}, g\right) \stackrel{U_{\nu}}{\rightarrow} \text { End } C^{\infty}\left(S^{m}\right),
$$

in the group and Lie algebra senses respectively. 
Remark 2.1. The extra summand of $m / 2$ above is the customary " $\rho$-shift": it follows from the last paragraph that an isometric and positivity preserving identification of $\mathfrak{a}^{*}$ with $\mathbb{R}$ identifies $\rho$ with $m / 2$. We put in the $\rho$-shift for ease of contact with the Lie theoretic literature, but its use is just a convention.

Note that since $l(K)$ and $l(\mathfrak{k})$ consist of isometric transformations and vector fields respectively,

$$
\Omega_{h}=1, \quad h \in l(K) ; \quad \omega_{X}=0, \quad X \in l(\mathfrak{k}) ;
$$

thus $\left.u_{\nu}\right|_{K}$ and $\left.U_{\nu}\right|_{\mathfrak{e}}$ are independent of $\nu$. We shall now show that the infinitesimal maps $U_{\nu}(X)$ stabilize $\mathscr{E}\left(S^{m}\right)$.

Lemma 2.2. Let $E_{j}$ be the space of $j$ th -order spherical harmonics on $S^{m} \cdot l(K)$ and $l(\mathfrak{k})$ stabilize each $E_{j}$, and

$$
\omega_{X} E_{j}, X E_{j} \subset E_{j-1} \oplus E_{j+1}, \quad X \in \imath(\mathfrak{s}),
$$

where by convention, $E_{-1}=0$. In particular, $U_{\nu}$ is a Lie algebra homomorphism from $\operatorname{cvf}\left(S^{m}, g\right)$ to End $\mathscr{E}\left(S^{m}\right)$.

Proof. $l(K)$ is the rotation group of $S^{m}$, and thus stabilizes each $E_{j}$. The assertions about $\mathfrak{s}$ are immediate once we note that in homogeneous coordinates $\left(y_{0}, \ldots, y_{m}\right)$ on $S^{m} \subset \mathbb{R}^{m+1}, L_{i, m+1}=\partial_{y_{i}}$; and that $\omega_{L_{i, m+1}}$ is the homogeneous coordinate function $y_{i}$, for $0 \leq i \leq m$.

Of course, the treatment just above is a very special case of a standard setup in semisimple Lie theory. In the general semisimple setting, and in the notation above, we can view $C^{\infty}(K / M)$ as the space of right- $M$-fixed vectors in $C^{\infty}(K)$,

$$
C^{\infty}(K / M) \simeq_{K}\left\{\varphi \in C^{\infty}(K) \mid \varphi(k m)=\varphi(k) \text {, all } k \in K, m \in M\right\} .
$$

Let $P=P_{\min }=M A N$. When we switch to the $G / P$ realization of $K / M$, we can choose the effect of $A$ in the corresponding right equivariance rule:

$$
\begin{aligned}
C^{\infty}(G / P) & \simeq_{K} C^{\infty}\left(\operatorname{Ind}_{P}^{G} 1 \otimes \lambda \otimes 1\right) \\
& =\left\{\psi \in C^{\infty}(G) \mid \psi(x m a n)=a^{-\lambda-\rho} \psi(x),\right. \\
& \text { all } x \in G, m \in M, a \in A, n \in N\} .
\end{aligned}
$$

Here, for $\lambda \in \mathfrak{a}^{*}$, we define $a^{\lambda}$ as $e^{\lambda(\log a)}$, using the fact that the exponential map is a diffeomorphism from $\mathfrak{a}$ to $A$. The notation $\operatorname{Ind}_{P}^{G} 1 \otimes \lambda \otimes 1$ indicates that we induce, from $P$ to $G$, the representation that acts trivially on $M$ and $N$, and by $a \mapsto a^{-\lambda-\rho}$ on $A . G$ and $\mathfrak{g}$ act on these spaces of equivariant functions via left multiplication to give homomorphisms

$$
\pi_{\lambda}: G \rightarrow \operatorname{Aut} C^{\infty}(G / P), \quad \Pi_{\lambda}: \mathfrak{g} \rightarrow \text { End } C^{\infty}(G / P),
$$

the second of which is the infinitesimal form of the first. The point is that when everything is unraveled, the homomorphisms $u_{\nu}$ of (2.6) are exactly the $\pi_{\nu \alpha}$, and similarly with $U, \Pi$ in place of $u, \pi$. To be more precise, if we use the letter $j$ for the identification $G / P \rightarrow S^{m}$, then

$$
\pi_{\nu \alpha}(x)\left(f_{\circ} j\right)=j \circ u_{\nu}(l(x)) f, \quad x \in G, f \in C^{\infty}\left(S^{m}\right) .
$$

We shall now do our work entirely in the elementary differential geometric setting, and suppress the identifications $l$ and $j$ in the notation.

We shall need some results that imply the uniqueness of intertwining operators; these are closely related to results on irreducibility, which we shall also state and prove. The driving result is the following. Let $u$ be the $\nu$-independent $K$-representation $u_{\nu}$. 
Lemma 2.3 (Cocycle irreducibility). For all $j \in \mathbb{N}$,

$$
\operatorname{Proj}_{E_{j \pm 1}} \omega(\mathfrak{s}) E_{j}=E_{j \pm 1},
$$

where $\omega(\mathfrak{s}):=\left\{\omega_{X} \mid X \in \mathfrak{s}\right\}$. As a result, the orbit $\mathscr{O}(f)$ of any nonzero $f \in E_{j}$, for any $j \in \mathbb{N}$, under the joint action of $u(K)$ and $\omega(\mathfrak{s})$, is $\mathscr{E}\left(S^{m}\right)$. In particular, $\mathscr{E}\left(S^{m}\right)$ has no nontrivial invariant subspace under this joint action. Proof. By the proof of Lemma 2.2, if $\left\{X_{i}\right\}_{i=0}^{m}$ is any orthonormal basis of $\mathfrak{s}$,

$$
\sum_{i=0}^{m} \omega_{X_{i}}^{2}=1
$$

(This sum is independent of the choice of orthonormal basis, and is the sum of squares of the homogeneous coordinate functions for the choice made above.) For each $j \in \mathbb{N}$, put

$$
t_{j}^{ \pm}=\left.\sum_{i=0}^{m} \operatorname{Proj}_{E_{j}} \omega_{X_{i}} \operatorname{Proj}_{E_{j \pm 1}} \omega_{X_{i}}\right|_{E_{j}} .
$$

The sum on the right is a $K$-endomorphism of $E_{j}$, and so is a scalar by Schur's Lemma. By (2.8), $t_{j}^{+}+t_{j}^{-}=1$. By the trace identity $\operatorname{tr} a b=\operatorname{tr} b a$,

$$
\left(\operatorname{dim} E_{j}\right) t_{j}^{+}=\left(\operatorname{dim} E_{j+1}\right) t_{j+1}^{-}, \quad j \in \mathbb{N} .
$$

This allows us to compute the $t_{j}^{ \pm}$inductively: since $t_{0}^{+}=1$ and

$$
\frac{\operatorname{dim} E_{j+1}}{\operatorname{dim} E_{j}}=\frac{(m+j-1)(m+2 j+1)}{(j+1)(m+2 j-1)},
$$

we get

$$
t_{j}^{+}=\frac{m+j-1}{m+2 j-1}, \quad t_{j}^{-}=\frac{j}{m+2 j-1} .
$$

In particular, all of these numbers except $t_{0}^{-}$are nonzero. Thus $\operatorname{Proj}_{E_{j \neq 1}} \omega(\mathfrak{s}) E_{j}$ is nonzero unless $j=0$ and the minus sign is chosen. But $X \otimes \varphi \mapsto \operatorname{Proj}_{E_{j \pm 1}} \omega_{X} \varphi$ is an $\mathrm{SO}(m)$ map $\mathfrak{s} \otimes E_{j} \rightarrow E_{j \pm 1}$, so Schur's Lemma gives (2.7).

For the statements about $\mathscr{O}(f)$, first note that since $\mathrm{SO}(m)$ is transitive on $E_{j}, \mathscr{O}(f) \supset E_{j}$. By $(2.7), \mathscr{O}(f) \supset E_{j-1} \oplus E_{j} \oplus E_{j+1} ;$ by induction $\mathscr{O}(f)=$ $\mathscr{E}\left(S^{m}\right)$. The $E_{j}$ are $\mathrm{SO}(m)$-invariant, so any invariant subspace is of the form $V=\bigoplus_{j \in B} E_{j}$ for some subset $B$ of the natural numbers $\mathbb{N}$. If $V \neq 0$, it has a nonzero $f$ in some $E_{j}$ as above, forcing it to be $\mathscr{E}\left(S^{m}\right)$.

The results we need really amount to irreducibility under some of the $(u(K)$, $\left.U_{\nu}(\mathfrak{s})\right)$. The above lemma replaces a generic irreducibility theorem of Bruhat [Bru], which implies irreducibility off a set of measure 0 in the $\mathfrak{a}_{\mathbb{C}}^{*}$ parameter. In our setting, all reducibility questions are settled by the cocycle $\omega$, since in a certain sense all the representations $U_{\nu}$ can be written in terms of $\omega$. We make this precise in the following.

Lemma 2.4. If $\Delta$ is the Laplacian on $S^{m}$, then

$$
\left[\Delta, \mu\left(\omega_{X}\right)\right]=2 U_{0}(X), \quad X \in \mathfrak{s} .
$$

Proof. We need only prove this for the basis elements $X=L_{i, m+1}, 0 \leq i \leq m$. By symmetry ( $K$-invariance and irreducibility of $\mathfrak{s}$ under $K$ ), we need only 
treat $Z=L_{0, m+1}$. For this, introduce the azimuthal angular coordinate $p$, i.e., that with

$$
\cos p=y_{0}=\omega_{Z} .
$$

Since $Z=(\sin p) \partial_{p}$, we have

$$
d y_{0}=-Z_{\mathrm{b}}
$$

where $b$ is the metric identification $T S^{m} \rightarrow T^{*} S^{m}$. But

$$
\left[\Delta, \mu\left(y_{0}\right)\right]=\Delta y_{0}-2 l\left(d y_{0}\right) d=\Delta y_{0}+2 l(Z) d=\Delta y_{0}+2 Z,
$$

where $l$ is interior multiplication. Since $y_{0} \in E_{1}$ and

$$
\left.\Delta\right|_{E_{j}}=\kappa_{j}=j(m-1+j),
$$

$\Delta y_{0}=m y_{0}$, so $\left[\Delta, \mu\left(y_{0}\right)\right]=2 Z+m y_{0}=2 U_{0}(Z)$, as desired.

Corollary 2.5. If $X \in \mathfrak{g}$,

$$
\begin{aligned}
& \left.\operatorname{Proj}_{E_{j+1}} U_{\nu}(X)\right|_{E_{j}}=\left.\frac{m+2 j+2 \nu}{2} \operatorname{Proj}_{E_{j+1}} \omega_{X}\right|_{E_{j}}, \\
& \left.\operatorname{Proj}_{E_{j-1}} U_{\nu}(X)\right|_{E_{j}}=\left.\frac{-m-2 j+2+2 \nu}{2} \operatorname{Proj}_{E_{j-1}} \omega_{X}\right|_{E_{j}} .
\end{aligned}
$$

If $m+2 j+2 \nu \neq 0$, then $\operatorname{Proj}_{E_{j+1}} U_{\nu}(\mathfrak{s}) E_{j}=E_{j+1}$. If $m+2 j-2-2 \nu \neq 0$, then $\operatorname{Proj}_{E_{j-1}} U_{\nu}(\mathfrak{s}) E_{j}=E_{j-1}$.

Proof. If $X \in \mathfrak{k}$, all terms in (2.11) vanish, so we may assume $X \in \mathfrak{s}$. Then (2.11) follows from Lemmas 2.3 and 2.4, together with the fact that $\kappa_{j+1}-\kappa_{j}=$ $m+2 j$.

Corollary 2.6 (Irreducibility). If $\pm \nu \notin m / 2+\mathbb{N}$, the assertions of Lemma 2.3 hold with $\left(u(K), U_{\nu}(\mathfrak{s})\right)$ in place of $(u(K), \omega(\mathfrak{s}))$.

Proof. This is immediate from Corollary 2.5 , once we note that the 0 coefficient there when $\nu=(m-2) / 2$ describes a map from $E_{0}$ to $E_{-1}$.

The $u_{\sqrt{-1} \lambda}$ for $\lambda \in \mathbb{R}$ are called the unitary principal series, and the $u_{\nu}$ for $\nu \in \mathbb{R},|\nu|<m / 2$ are the complementary series. In the course of computing the intertwining operators for the principal series, we shall be able to explain how this terminology came about (Remark 2.9 below). More importantly, we shall make contact with the $S^{m}$ realizations of the conformally covariant operators $P_{n}$, and with Lieb's best constant for the Hardy-Littlewood-Sobolev inequality.

Definition 2.7. A linear operator $T$ on $\mathscr{E}\left(S^{m}\right)$ is intertwining of bidegree $(\nu, \sigma)$ $\in \mathbb{C}^{2}$ if $T$ commutes with $u(K)$, and $T U_{\nu}(X)=U_{\sigma}(X) T$ for all $X \in \mathfrak{s}$.

Intertwining in this $(\mathfrak{g}, K)$-module sense is equivalent to intertwining in an appropriately formulated group sense; for us, this will be easiest to see from the concrete form of the intertwinors. Note that a conformally covariant differential operator in the sense of $(1.2,1.3)$, when evaluated in the standard $S^{m}$, is intertwining of bidegree $\left(a-\frac{m}{2}, b-\frac{m}{2}\right)$. Schur's Lemma, together with the fact that the $E_{j}$ are inequivalent irreducible $K$-modules, shows that each intertwinor $T$ is diagonalized by the decomposition $\mathscr{E}\left(S^{m}\right)=\bigoplus E_{j}$. That is, we can identify $T$ with a list of eigenvalues $\mu_{j} \in \mathbb{C},\left.T\right|_{E_{j}}=\mu_{j}$. Corollary 
2.5 shows that we can construct a $T$ of bidegree $(\sigma, \nu)$ by finding a list $\left\{\mu_{j}\right\}$ satisfying

$$
\begin{aligned}
(m+2 j+2 \nu) \mu_{j+1} & =(m+2 j+2 \sigma) \mu_{j}, \\
(-m-2 j+2+2 \nu) \mu_{j-1} & =(-m-2 j+2+2 \sigma) \mu_{j} .
\end{aligned}
$$

Apart from trivialities, there is no such list unless the consistency constraint $\sigma=-\nu$ is satisfied. When $-\nu \notin \frac{m}{2}+\mathbb{N}$, however, there is a unique such list (up to a constant factor) giving an intertwinor of bidegree $(-\nu, \nu)$, that being

$$
\mu_{j}^{\nu}=\frac{\Gamma\left(\frac{m}{2}+j+\nu\right)}{\Gamma\left(\frac{m}{2}+j-\nu\right)} .
$$

If $\nu \in \frac{m}{2}+\mathbb{N}$, this formula is to be interpreted in the sense of analytic continuation; that is, $\mu_{j}^{\nu}=0$ if $\nu \in \frac{m}{2}+j+\mathbb{N}$. By (2.10),

$$
\mu_{j}^{\nu}=\left.\frac{\Gamma\left(B+\nu+\frac{1}{2}\right)}{\Gamma\left(B-\nu+\frac{1}{2}\right)}\right|_{E_{j}},
$$

where

$$
B=\sqrt{\Delta+\left(\frac{m-1}{2}\right)^{2}} .
$$

Note that

$$
\left.B\right|_{E_{j}}=j+\frac{m-1}{2}
$$

We can now state:

Theorem 2.8. (a) Aside from multiples of the identity, all intertwinors have bidegree $(-\nu, \nu)$ for some $\nu \in \mathbb{C}$. If $-\nu \notin \frac{m}{2}+\mathbb{N}$, the operator

$$
A_{2 \nu}:=\Gamma\left(B+\nu+\frac{1}{2}\right) / \Gamma\left(B-\nu+\frac{1}{2}\right)
$$

is the unique intertwinor of bidegree $(-\nu, \nu)$, up to a constant factor. If $\nu \notin$ $\frac{m}{2}+\mathbb{N}$, the operator

$$
\tilde{A}_{2 \nu}:=\frac{\Gamma\left(\frac{m}{2}-\nu\right) \Gamma\left(B+\nu+\frac{1}{2}\right)}{\Gamma\left(\frac{m}{2}+\nu\right) \Gamma\left(B-\nu+\frac{1}{2}\right)}
$$

is the unique such intertwinor, up to a constant factor.

(b) If $-\nu \in \frac{m}{2}+\mathbb{N}$, then $\tilde{A}_{2 \nu}$ is a finite rank operator on $\mathscr{E}\left(S^{m}\right)$. For $-\nu \in \mathbb{R} \backslash\left(\frac{m}{2}+\mathbb{N}\right), A_{2 \nu}$ extends to a bounded operator of loss $2 \nu$ between Sobolev classes; that is $A_{2 \nu}: L_{k}^{2}\left(S^{m}\right) \rightarrow L_{k-2 \nu}^{2}\left(S^{m}\right)$ for all $k \in \mathbb{R}$. Denoting the space of operators with loss $l$ by $\Psi_{l}\left(S^{m}\right)$, we have that $A_{2 \nu}$ lies in the coset $\Delta^{\nu}+\Psi_{2 \nu-1}\left(S^{m}\right) \in \Psi_{2 \nu}\left(S^{m}\right) / \Psi_{2 \nu-1}\left(S^{m}\right)$.

(c) For $-\nu \in \mathbb{R} \backslash\left(\frac{m}{2}+\mathbb{N}\right), A_{2 \nu}$ is $G$ intertwining:

$$
A_{2 \nu} u_{-\nu}(h)=u_{\nu}(h) A_{2 \nu} \quad \text { on } C^{\infty}\left(S^{m}\right) \text { for all } h \in G .
$$

The same statement holds for $\tilde{A}_{2 \nu}$ if $\nu \in \mathbb{R} \backslash\left(\frac{m}{2}+\mathbb{N}\right)$.

(d) For $-\nu \in \mathbb{R} \backslash\left(\frac{m}{2}+\mathbb{N}\right), A_{2 \nu}$ is symmetric: if $\varphi_{i}$ is in the Sobolev class $L_{k_{i}}^{2}\left(S^{m}\right), i=1,2$, with $k_{1}+k_{2}=2 \nu$, then $\left(\varphi_{1}, A_{2 \nu} \varphi_{2}\right)=\left(A_{2 \nu} \varphi_{1}, \varphi_{2}\right)$, where $(\cdot, \cdot)$ is the extension of the (sesquilinear) $L^{2}$ inner product to a pairing of $C^{\infty}$ functions with distributions. The same is true of $\tilde{A}_{2 \nu}$ if $\nu \in \mathbb{R} \backslash\left(\frac{m}{2}+\mathbb{N}\right)$. 
(e) If $\nu \in \mathbb{R}$ and $|\nu|<m / 2$, then $A_{2 \nu}$ is a positive operator: if $\varphi \in L_{\nu}^{2}\left(S^{m}\right)$, then $\left(A_{2 \nu} \varphi, \varphi\right) \geq 0$, with equality if and only if $\varphi=0$.

(f) If $(m, n)$ and $P_{n}$ are as in Theorem 1.1, then on $S^{m}$ with its standard metric $g_{0}$,

$$
\left(P_{n}\right)_{0}=A_{n}=\prod_{\alpha=1}^{n / 2}\left\{\left(B+\alpha-\frac{1}{2}\right)\left(B-\alpha+\frac{1}{2}\right)\right\}, \quad\left(Q_{n}\right)_{0}=\frac{\Gamma\left(\frac{m+n}{2}\right)}{\Gamma\left(\frac{m-n+2}{2}\right)} .
$$

In particular, $\left(Q_{m}\right)_{0}=\Gamma(m)$. As a result, if $\omega \in C^{\infty}\left(S^{m}\right)$, then in the metric $g_{\omega}=e^{2 \omega} g_{0}$,

$$
\left(P_{n}\right)_{\omega}=e^{-\frac{m+n}{2} \omega} A_{n} \mu\left(e^{\frac{m-n}{2} \omega}\right) .
$$

If $n \neq m$,

$$
\left(Q_{n} d v\right)_{\omega}=e^{\beta \omega}\left\{\frac{\Gamma\left(\frac{m+n}{2}\right)}{\Gamma\left(\frac{m-n+2}{2}\right)}+A_{n} \frac{e^{\beta \omega}-1}{\beta}\right\}(d v)_{0},
$$

where $\beta=(m-n) / 2$; and

$$
\left(Q_{m} d v\right)_{\omega}=\left(A_{m} \omega+\left(Q_{m}\right)_{0}\right)(d v)_{0} .
$$

Proof. (a) is immediate from (2.15) and the discussion above. Note that $A_{2 \nu}$ and $\tilde{A}_{2 \nu}$ agree (are nonzero constant multiples of one another) where both are defined. If $-\nu \in \frac{m}{2}+\mathbb{N}$, the factor $\Gamma\left(B+\nu+\frac{1}{2}\right) / \Gamma\left(\frac{m}{2}+\nu\right)$ in $\tilde{A}_{2 \nu}$ annihilates $E_{j}$ for $j \geq-\nu-\frac{m}{2}+1$; this proves the finite rank assertion. By (2.10), any operator $T$ with $\left.T\right|_{E_{j}}=\lambda_{j} \sim$ const $\cdot j^{\delta}$ as $j \rightarrow \infty$ extends to a bounded operator $L_{k}^{2} \rightarrow L_{k-\delta}^{2}$ for all $k \in \mathbb{R}$. But by Stirling's formula, $\mu_{j}^{\nu} \sim j^{2 \nu}+c j^{2 \nu-1}$ for some constant $c$; this proves the rest of (b). (c) is proved formally, on a dense set of $h \in G$, by computing that

$$
(d / d \varepsilon)\left\{u_{\nu}\left(h_{-\varepsilon}\right) A_{2 \nu} u_{-\nu}\left(h_{\varepsilon}\right)\right\}=0
$$

as a consequence of the $\mathfrak{g}$ intertwining relation, where $h_{\varepsilon}$ is a local oneparameter group in $G$. By part (b), this calculation makes sense in a space of bounded operators between appropriate Sobolev spaces and gives (2.16) as an operator identity; by the Sobolev Lemma, (2.16) is true in the elementary sense. The same argument works for the finite rank operators in part (b). (d) is true formally because the eigenvalues of $A_{2 \nu}$ and $\tilde{A}_{2 \nu}$ are real; the orders of the Sobolev spaces involved are computed from (b). (e) is immediate from the eigenvalue list (2.13).

For (f), first note that by $(2.4)$ and Remark $2.1,\left(P_{n}\right)_{0}$ must be intertwining of bidegree $(-n / 2, n / 2)$ on $\left(S^{m}, g_{0}\right)$. Indeed, by universality, $P_{n}$ is $K$ intertwining; by the infinitesimal conformal covariance relation applied to the functions $\omega_{X}, X \in \operatorname{cvf}\left(S^{m}, g_{0}\right), P_{n}$ must be $\mathfrak{g}$ intertwining. Since $A_{n}$ is the only intertwinor of bidegree $(-n / 2, n / 2)$ with the correct leading symbol (that of $\left.\Delta^{n / 2}\right)$, it follows that $\left(P_{n}\right)_{0}=A_{n}$. The formula for $\left(P_{n}\right)_{0}=A_{n}$ now follows from part $(\mathrm{a})$, and the formula for $\left(P_{n}\right)_{\omega}$ follows from conformal covariance. The formulas for $Q_{n}$ are derived by applying those for $P_{n}$ to the constant function 1 , except when $n=m$. The formula for $Q_{m}$ follows from (1.14).

Remark 2.9. It is easily seen that the $u_{\sqrt{-1} \lambda}$ for $\lambda \in \mathbb{R}$ are unitary in the $L^{2}$ inner product; these representations make up the unitary principal series 
mentioned before, but will not be of direct interest to us. The inner product $\left(\cdot, A_{2 \nu} \cdot\right)$ is invariant for $u_{-\nu}$ whenever $-\nu \in \mathbb{R} \backslash\left(\frac{m}{2}+\mathbb{N}\right)$, and is positive definite if and only if $|\nu|<m / 2$. In fact, Theorem 2.8 shows that if $\nu \in(-m / 2, m / 2)$, the norm corresponding to the inner product $\left(\cdot, A_{2 \nu} \cdot\right)$ defines the Sobolev class $L_{\nu}^{2}$. The $\left(L_{\nu}^{2}\left(S^{m}\right), u_{-\nu}\right)$ for $\nu \in(-m / 2, m / 2)$ are the complementary series mentioned above; they are directly involved in what follows.

\section{CONFORMAL COVARIANCE ON $\mathbb{R}^{m}$, AND THE INEQUAlities OF LIEB AND BECKNER}

In the last section, we established an intertwining property, equivalent to covariance under multiplication of the metric by some special conformal factors, for the operators

$$
A_{2 \nu} \in \Delta_{S^{m}}^{\nu}+\Psi_{2 \nu-1}\left(S^{m}\right), \quad-\nu \notin \frac{m}{2}+\mathbb{N},
$$

where $\Psi_{d}\left(S^{m}\right)$ is the space of operators of loss $d$ described in Theorem $2.8(\mathrm{~b})$. The operators $P_{n}$ of Theorem 1.1, when evaluated on $S^{m}$, are special cases. In this section, we shall use stereographic projection to view the intertwinors $A_{2 \nu}$ on $\mathbb{R}^{m}$ with its standard metric. Not surprisingly, they turn out to be exactly the operators $\Delta_{\mathbb{R}^{m}}^{\nu}$. For the complementary series range of $\nu$, viz. $|\nu|<m / 2$, these operators are given by integral kernels; in fact exactly those integral kernels estimated by the Lieb inequalities. This circumstance allowed Beckner to give a simple expression for these inequalities on $S^{m}$, using facts about spherical harmonics and Gegenbauer polynomials. Here we shall give an explanation that does not depend on these technical devices, but only on group invariance.

For ease of comparison, we follow Beckner's notation for the time being. Accordingly, let $d \xi$ be the normalized measure on the standard sphere, so that $\int_{S^{m}} d \xi=1$. We view $S^{m}$ as the unit sphere in a copy of $\mathbb{R}^{m+1}$ with coordinate function $\xi=(u, s) \in \mathbb{R}^{m} \times \mathbb{R}$. Our convention for the stereographic projection will be as follows: we identify $\mathbb{R}^{m}$, whose coordinate will be called $x$, with the complement $S^{m} \backslash(0,-1)$ of the south pole via

$$
x=\frac{u}{1+s}, \quad u=\frac{2 x}{1+r^{2}}, \quad s=\frac{1-r^{2}}{1+r^{2}}:=\cos p, \quad \alpha:=|u|=\sin p .
$$

Here $r=|x|$, and $p$ is the azimuthal angle mentioned in the proof of Lemma 2.4. The standard metrics are related by

$$
g_{\mathbb{R}^{m}}=\Phi^{2} g_{S^{m}}, \quad \Phi=\frac{1}{2}\left(1+r^{2}\right)=\frac{1}{1+s} .
$$

If $d \xi^{\prime}$ is the usual, unnormalized Riemannian measure on $S^{m},(3.1)$ implies that

$$
d x=\left(\frac{1+r^{2}}{2}\right)^{m} d \xi^{\prime}=\left(\frac{1+r^{2}}{2}\right)^{m} v_{m} d \xi
$$

where

$$
v_{m}=\frac{2 \pi^{\frac{m+1}{2}}}{\Gamma\left(\frac{m+1}{2}\right)}=\frac{(4 \pi)^{\frac{m}{2}} \Gamma\left(\frac{m}{2}\right)}{\Gamma(m)}
$$

is the volume of $\left(S^{m}, d \xi^{\prime}\right)$.

The stereographic projection, like the intertwining principle of the last section, is a special case of a general setup in semisimple theory. Let $\bar{N}=\exp \overline{\mathfrak{n}}$, 
and let $o$ be the identity coset in $G / M A N$. There is a natural diffeomorphic injection $\bar{N} \rightarrow G / M A N$, given by $\bar{n} \mapsto \bar{n} \cdot O$. In our situation, $\bar{N}$ is naturally identified with $\mathbb{R}^{m}$, and as shown in the last section, $G / M A N$ is naturally $S^{m}$. We omit the details of this approach, noting only its potential usefulness in future applications to, for example, CR geometry (replacing $\mathrm{SO}_{0}(m+1,1)$ by $\mathrm{SU}(m+1,1))$.

One of the convenient aspects of having both the $S^{m}$ and $\mathbb{R}^{m}$ pictures is that conformal covariance of a pseudo-differential operator $T$ is easy to check, given some explicit formula for $T$. The full conformal group $\mathrm{O}(m+1,1)$ of the sphere, when realized in the $\mathbb{R}^{m}$ picture, is generated by the group $\mathrm{O}(m)$ of origin-fixing isometries, the translation group $\mathbb{R}^{m}$, the dilations $\delta_{c}: x \mapsto c x$ for $0<c \in \mathbb{R}$, and the inversion in the unit sphere, $Q: x \rightarrow x / r^{2}$. The inversion is singular at $x=0$; this is the reason why one must compactify to get an "honest" group action. In the $S^{m}$ picture, $Q:(u, s) \mapsto(u,-s)$ is an isometry. This gives rise to the following useful principle:

Proposition 3.1. Suppose $S$ (resp. $T$ ) is a linear transformation on $C^{\infty}\left(S^{m}\right)$ (resp. $\left.C^{\infty}\left(\mathbb{R}^{m}\right)\right)$, with

$$
T=\Phi^{-b} S \mu\left(\Phi^{a}\right)
$$

for some $a, b \in \mathbb{C}$, where $\Phi$ is as in (3.1). If $S$ is isometry invariant on $S^{m}$, $T$ is isometry invariant on $\mathbb{R}^{m}$, and the dilational behavior of $T$ is

$$
T\left(\delta_{c} \cdot f\right)=c^{a-b} \delta_{c} \cdot(T f)
$$

for all $c>0$ and $f \in C^{\infty}\left(\mathbb{R}^{m}\right)$, then $S$ is intertwining of bidegree $(a-m / 2, b-$ $m / 2)$ on $S^{m}$, and

$$
T\left(r^{-2 a} Q \cdot f\right)=r^{-2 b} Q \cdot(T f)
$$

for all $f \in C^{\infty}\left(\mathbb{R}^{m}\right)$.

The proof is based on the following observation:

Lemma 3.2. Let $g$ and $\bar{g}=\Phi^{2} g$ be conformally related metrics on a manifold $M, 0<\Phi \in C^{\infty}(M)$. If $h \in \operatorname{ctran}(M, g), h \cdot g=\Omega_{h}^{2} g$, then $h \in \operatorname{ctran}(M, \bar{g})$ and $h \cdot \bar{g}=\Xi_{h}^{2} g$ with $\Phi \Xi_{h}=(h \cdot \Phi) \Omega_{h}$. Fix $h \in \operatorname{ctran}(M, g)=\operatorname{ctran}(M, \bar{g})$; suppose that $S$ is a linear transformation on $C^{\infty}(M)$ with the intertwining property

$$
S\left(\Omega_{h}^{a} h \cdot \varphi\right)=\Omega_{h}^{b} h \cdot(S \varphi), \quad \text { all } \varphi \in C^{\infty}(M)
$$

for some $a, b \in \mathbb{C}$; and let $T=\Phi^{-b} S \mu\left(\Phi^{a}\right)$. Then $T$ has the intertwining property

$$
T\left(\Xi_{h}^{a} h \cdot f\right)=\Xi_{h}^{b} h \cdot(T f), \quad \text { all } f \in C^{\infty}(M) .
$$

Proof. This is immediate from the identities $h \cdot(\varphi \psi)=(h \cdot \varphi)(h \cdot \psi)$ for all $\varphi, \psi \in C^{\infty}(M)$, and $h \cdot \Phi^{a}=(h \cdot \Phi)^{a}$ for all $0<\Phi \in C^{\infty}(M)$ and $a \in \mathbb{C}$.

Proof of Proposition 3.1. We apply the lemma in the case where $g=g_{S^{m}}$ and $\bar{g}=g_{\mathbb{R}^{m}}$. Because of the singularity of the inversion $Q$ on $\mathbb{R}^{m}$, the manifold $M$ can be taken to be $S^{m}$ minus at most two points, or conformally equivalently, $\mathbb{R}^{m}$ minus at most one point, the puncture sets depending on the conformal transformation considered. The proposition follows from the fact 
that the conformal transformations are generated by the isometries on $\mathbb{R}^{m}$ and $S^{m}$, together with the dilations on $\mathbb{R}^{m}$, once we note that (3.3) is the conformal covariance relation for dilations $\left(\Xi_{\delta_{c}}=c\right)$, and (3.4) is the conformal covariance relation for $Q\left(\Xi_{Q}=r^{-2}\right)$.

Consider now the convolution operators $r^{-(m-2 \nu)} *$ on $\mathbb{R}^{m}$ for $\nu \in(0, m / 2]$; in detail,

$$
\left(r^{-(m-2 \nu)} * f\right)(y)=\int_{\mathbb{R}^{m}} f(x)|x-y|^{-(m-2 \nu)} d x .
$$

$r^{-(m-2 \nu)} *$ can be naturally viewed as a bounded operator from $L^{p}\left(\mathbb{R}^{m}\right)$ to its dual $L^{q}\left(\mathbb{R}^{m}\right)$, where $p=2 m /(m+2 \nu)$ and $q=2 m /(m-2 \nu)$. We would also like to view $r^{-(m-2 \nu)} *$ as an operator between $L^{2}$ Sobolev spaces, and make contact with the theory of the principal series. The philosophy is that up to a constant factor, $r^{-(m-2 \nu)_{*}}$ is an inverse for $\Delta^{\nu}$; we shall not develop this thinking precisely, but rather move to the compact setting of the sphere, where all is straightforward. The numbers $p, q, \nu$ are related by the commutative diagram (3.5), in which the maps $l$ are borderline Sobolev embeddings, and the map on the right is bounded as a consequence of the boundedness of the other three maps. The norms on all spaces are conformally invariant; on the top line, in the representation $u_{-\nu}$, and on the bottom line, in the representation $u_{\nu}$. For the $L^{2}$ Sobolev spaces, this is a consequence of Remark 2.9; for $L^{p}$ and $L^{q}$, it follows upon a change of variable once we notice that $h \cdot d \xi=\Omega_{h}^{m} d \xi$ for $h \in \operatorname{ctran}\left(S^{m}, g\right)$.

$$
\begin{array}{cc}
L_{\nu}^{2}\left(S^{m}\right) & l \\
\uparrow A_{-2 \nu} & L^{q}\left(S^{m}\right) \\
L_{-\nu}^{2}\left(S^{m}\right) & \longleftarrow A_{-2 \nu} \\
&
\end{array}
$$

Using hard analytic symmetrization arguments, Lieb showed that:

Theorem 3.3 [Lie]. If $\nu \in(0, m / 2]$,

$$
\max _{\mathbb{P} L^{p}\left(\mathbb{R}^{m}\right)} \frac{\left|\left\langle r^{-(m-2 \nu)} * f, g\right\rangle\right|}{\|f\|_{p}\|g\|_{p}}
$$

is attained exactly at

$$
[f]=[g]=\left[\Xi_{h}^{m / 2+\nu} h \cdot \Phi^{-(m / 2+\nu)}\right],
$$

where $h$ is a conformal transformation on $\left(\mathbb{R}^{m}, \bar{g}\right)$ as above (i.e., $h$ may have a point singularity), and $h \cdot \bar{g}=\Xi_{h}^{2} g$. Here $\mathbb{P} L^{p}\left(\mathbb{R}^{m}\right)$ is the projective space on $L^{p}\left(\mathbb{R}^{m}\right),[\cdot]$ is the projective equivalence class, and $\langle\cdot, \cdot\rangle$ is the pairing of $L^{q}\left(\mathbb{R}^{m}, d x\right)$ and $L^{p}\left(\mathbb{R}^{m}, d x\right)$.

In particular, the maximum is attained at

$$
f=g=\left(1+r^{2}\right)^{-(m / 2+\nu)} .
$$

In the $\Omega, \Xi$ notation of Lemma 3.2, again taking $g=g_{S^{m}}, \bar{g}=g_{\mathbb{R}^{m}}$, we can rewrite (3.6) as

$$
[f]=[g]=\left[\left(\Omega_{h} / \Phi\right)^{m / 2+\nu}\right]
$$


Consider the correspondences

$$
f \stackrel{(p)}{\longleftrightarrow} \varphi=\Phi^{m / 2+\nu} f, \quad k \stackrel{(q)}{\longleftrightarrow} \kappa=\Phi^{m / 2-\nu} k
$$

between measurable functions $f, k$ on $\mathbb{R}^{m}$ and $\varphi, \kappa$ on $S^{m}$. Since $d x=$ $\Phi^{m} d \xi^{\prime}=v_{m} \Phi^{m} d \xi$

$$
\begin{aligned}
\|f\|_{L^{p}\left(\mathbb{R}^{m}, d x\right)} & =v_{m}^{1 / p}\|\varphi\|_{L^{p}\left(S^{m}, d \xi\right)}, \\
\int_{\mathbb{R}^{m}} k(x) f(x) d x & =v_{m} \int_{S^{m}} \kappa(\xi) \varphi(\xi) d \xi .
\end{aligned}
$$

To transfer Lieb's inequality, we need to write the operator $r^{-(m-2 \nu)} *$ in the spherical setting. Now the distance between $\xi, \eta \in S^{m}$ in the ambient $\mathbb{R}^{m+1}$ is

$$
|\xi-\eta|^{2}=\{\Phi(x) \Phi(y)\}^{-1}|x-y|^{2}
$$

where $\xi$ corresponds to $x$ and $\eta$ to $y$ under the stereographic projection. To make contact with Lemma 3.2 , let $T=r^{-(m-2 \nu)_{*}}$; then

$$
\begin{aligned}
(T f)(y) & =\left(T\left(\Phi^{\nu-m / 2} \varphi\right)\right)(\eta) \\
& =v_{m} \int_{S^{m}} \Phi(\xi)^{\nu-m / 2} \varphi(\xi)(\Phi(\xi) \Phi(\eta))^{-(m / 2-\nu)}|\xi-\eta|^{-(m-2 \nu)} \Phi(\xi)^{m} d \xi \\
& =v_{m} \Phi(\eta)^{-(m / 2-\nu)} \int_{S^{m}} \varphi(\xi)|\xi-\eta|^{-(m-2 \nu)} d \xi \\
& :=\Phi(\eta)^{-(m / 2-\nu)}(S \varphi)(\eta) .
\end{aligned}
$$

$T$ and $S$ are manifestly isometry invariant on $\mathbb{R}^{m}$ and $S^{m}$ respectively. By translation invariance, the dilational behavior of $T$ can be detected at a single point:

$$
\begin{aligned}
\left(T\left(\delta_{c} \cdot f\right)\right)(0) & =\int_{\mathbb{R}^{m}} f\left(c^{-1} x\right)|x|^{-(m-2 \nu)} d x=\int_{\mathbb{R}^{m}} f(y)(c|y|)^{-(m-2 \nu)} c^{m} d y \\
& =c^{2 \nu}(T f)(0),
\end{aligned}
$$

so $T\left(\delta_{c} \cdot f\right)=c^{2 \nu} \delta_{c} \cdot(T f)$. Thus by Proposition $3.1, S$ is intertwining of bidegree $(\nu,-\nu)$. By the uniqueness result Theorem 2.8(a), we must have

$$
S=b \tilde{A}_{-2 \nu}=b \frac{\Gamma\left(\frac{m}{2}+\nu\right) \Gamma\left(B-\nu+\frac{1}{2}\right)}{\Gamma\left(\frac{m}{2}-\nu\right) \Gamma\left(B+\nu+\frac{1}{2}\right)}
$$

for some nonzero constant $b$. In fact, since $S$ and $\tilde{A}_{-2 \nu}$ are both nonnegative, $b>0$. Putting all this together, we get

$$
\frac{\left|\left\langle\tilde{A}_{-2 \nu} \varphi, \psi\right\rangle_{\left(S^{m}, d \xi\right)}\right|}{\|\varphi\|_{L^{p}\left(S^{m}\right)}\|\psi\|_{L^{p}\left(S^{m}\right)}}=b^{-1} v_{m}^{2 \nu / m} \frac{\left|\left\langle r^{-(m-2 \nu)} * f, g\right\rangle_{\left(\mathbb{R}^{m}, d x\right)}\right|}{\|f\|_{L^{p}\left(\mathbb{R}^{m}, d x\right)}\|g\|_{L^{p}\left(\mathbb{R}^{m}, d x\right)}},
$$

where $f \stackrel{(p)}{\leftrightarrow} \varphi$ and $g \stackrel{(p)}{\leftrightarrow} \psi$ as above. Thus we can conclude:

Theorem 3.4 [Bec]. If $\nu \in(0, m / 2]$,

$$
\max _{\mathbb{P} L^{p}\left(S^{m}, d \xi\right)} \frac{\left|\left\langle\tilde{A}_{-2 \nu} \varphi, \psi\right\rangle_{\left(S^{m}, d \xi\right)}\right|}{\|\varphi\|_{L^{p}\left(S^{m}, d \xi\right)}\|\psi\|_{L^{p}\left(S^{m}, d \xi\right)}}
$$


is attained exactly for

$$
[\varphi]=[\psi]=\left[\Omega_{h}^{m / 2+\nu}\right], \quad h \in \operatorname{ctran}\left(S^{m}, g\right) .
$$

In particular, one of the extremal functions is 1 , so the maximum value is 1. The endpoint $\nu=0$, missing in Lieb's theorem, can be restored here: for $\nu=0, p=2$ and $\tilde{A}_{2 \nu}$ is the identity, so the functional is maximized whenever $[\varphi]=[\psi]$. This is a qualitative difference from the situation for $\nu \in(0, m / 2]$, where there is a finite-parameter set of minimizers.

It has turned out that an inequality dual to Theorem 3.4, as well as a generalized Moser-Trudinger inequality obtained by taking the dual of an endpoint derivative of Theorem 3.4, are crucial to the estimation of functional determinants of natural differential operators on $S^{2}$ and $S^{4}$ [On, OPS1-2, BØ3, BCY]. The above inequalities were derived in detail to show the power of the present group-invariance machinery, which replaces Beckner's use of technical devices like Gegenbauer polynomials and the Funk-Hecke formula. For the following inequalities, we refer to Beckner's arguments deriving them from Theorem 3.4. The geometric interpretations included in our statements of Corollaries 3.6 and 3.8 below are partially indicated in [Bec].

Theorem 3.5 (The Sobolev embedding $\left.L_{\nu}^{2} \hookrightarrow L^{2 m /(m-2 \nu)} \quad[\mathrm{Bec}]\right)$. If $\nu \in[0$, $m / 2)$, with notation as above,

$$
\min _{\mathbb{P} L^{q}\left(S^{m}, d \xi\right)} \frac{\left|\left\langle\tilde{A}_{2 \nu} \kappa, \lambda\right\rangle_{\left(S^{m}, d \xi\right)}\right|}{\|\kappa\|_{L^{q}\left(S^{m}, d \xi\right)}\|\lambda\|_{L^{q}\left(S^{m}, d \xi\right)}}
$$

is attained exactly for

$$
[\kappa]=[\lambda]=\left[\Omega_{h}^{m / 2-\nu}\right], \quad h \in \operatorname{ctran}\left(S^{m}, g\right) .
$$

Corollary 3.6. (a) If $\nu<m / 2$ is a positive integer and $n=2 \nu$,

$$
\begin{aligned}
\|\kappa\|_{L^{2 m /(m-n)}\left(S^{m}, d \xi\right)}^{2} & \leq \frac{\Gamma\left(\frac{m-n}{2}\right)}{\Gamma\left(\frac{m+n}{2}\right)}\left(A_{n} \kappa, \kappa\right)_{L^{2}\left(S^{m}, d \xi\right)} \\
& =\left(\beta\left(Q_{n}\right)_{0}\right)^{-1}\left(\left(P_{n}\right)_{0} \kappa, \kappa\right)_{L^{2}\left(S^{m}, d \xi\right)},
\end{aligned}
$$

with equality if and only if $[\kappa]=\left[\Omega_{h}^{\beta}\right], h \in \operatorname{ctran}\left(S^{m}, g\right)$, where $\beta=(m-n) / 2$.

(b) If $(m, n)$ is as in part $(\mathbf{a})$,

$$
\left\|\left(Q_{n}\right)_{\omega}\right\|_{L^{m / n}\left(S^{m},(d \xi)_{\omega}\right)}
$$

is minimized exactly when $\left[e^{\omega}\right]=\left[\Omega_{h}\right], h \in \operatorname{ctran}\left(S^{m}, g\right)$. Here $(d \xi)_{\omega}=$ $(d v)_{\omega} / v_{m}$, where $(d v)_{\omega}$ is the Riemannian measure of the metric $e^{2 \omega} g$.

Proof of Corollary 3.6. The transition from Theorem 3.5 to part (a) is made via Theorem 2.8. Part (b) can then be proved by an argument of Paul Yang. Let $c=\beta\left(Q_{n}\right)_{0}, q=m / \beta=2 m /(m-n), f=e^{\beta \omega}$, and $T=\left(P_{n}^{0}\right)_{0}$; then

$$
c\|f\|_{q}^{2} \leq \int_{S^{m}} f(T+c) f d \xi \leq\left\|f^{2}\right\|_{q / 2}\left\|\frac{T f}{f}+c\right\|_{q /(q-2)}
$$

by Hölder's inequality. Here $\|\cdot\|_{p}$ is the norm in $L^{p}\left(S^{m}, d \xi\right)$. Since $\|f\|_{q}^{2}=$ $\left\|f^{2}\right\|_{q / 2}$, this is the desired result. 
Corollary 3.6 gives an interpretation of the Sobolev embedding $L_{n / 2}^{2} \hookrightarrow$ $L^{2 m /(m-n)}$ in terms of local invariants which is similar to the interpretation of the Yamabe functional in terms of the total scalar curvature.

Theorem 3.7 (The embedding $L_{m / 2}^{2} \hookrightarrow e^{L}$ [Bec]). Let $\bar{\omega}=\int_{S^{m}} \omega d \xi$ be the mean value of $\omega \in C^{\infty}\left(S^{m}\right)$. Then

$$
\log \int_{S^{m}} e^{m(\omega-\bar{\omega})} d \xi \leq \frac{m}{2 \Gamma(m)}\left(P_{m} \omega, \omega\right)_{L^{2}\left(S^{m}, d \xi\right)},
$$

with equality if and only if

$$
\left[e^{\omega}\right]=\left[\Omega_{h}\right], \quad h \in \operatorname{ctran}\left(S^{m}, g\right) .
$$

Corollary 3.8 .

$$
\left(Q_{m}\right)_{0} \log \int_{S^{m}} e^{m \omega} d \xi \leq \frac{m}{2} \int_{S^{m}} \omega\left\{\left(Q_{m} d \xi\right)_{\omega}+\left(Q_{m} d \xi\right)_{0}\right\},
$$

with equality if and only if (3.9) holds. Under the volume-preservation restriction $\int_{S^{m}}(d \xi)_{\omega}=1$,

$$
0 \leq \frac{m}{2} \int_{S^{m}} \omega\left\{\left(Q_{m} d \xi\right)_{\omega}+\left(Q_{m} d \xi\right)_{0}\right\},
$$

with equality if and only if $e^{\omega}=\Omega_{h}$ for some $h \in \operatorname{ctran}\left(S^{m}, g\right)$.

Note that the invariance of the right side of (3.8) under scaling $(\omega \mapsto \omega+$ const) follows from the fact that $P_{m}$ is formally self-adjoint and annihilates constants in the conformally flat case.

Proof of Corollary 3.8. We use (1.14) to write (3.8) in the form

$$
\left(Q_{m}\right)_{0}\left(\log \int_{S^{m}} e^{m \omega} d \xi-m \bar{\omega}\right) \leq \frac{m}{2}\left\{\int_{S^{m}} \omega\left(Q_{m} d v\right)_{\omega} d \xi-\left(Q_{m}\right)_{0} \bar{\omega}\right\},
$$

with equality if and only if (3.9) holds. This is equivalent to

$$
\left(Q_{m}\right)_{0} \log \int_{S^{m}} e^{m \omega} d \xi \leq \frac{m}{2} \int_{S^{m}} \omega\left\{\left(Q_{m} d \xi\right)_{\omega}+\left(Q_{m} d \xi\right)_{0}\right\},
$$

with equality if and only if (3.9) holds. Since $\int_{S^{m}}(d \xi)_{\omega}=\int_{S^{m}} e^{m \omega} d \xi$, we get (3.11) for volume 1 conformal metrics. To see when equality holds in (3.11), we just have to note that metrics corresponding to $e^{\omega}=\Omega_{h}$ have volume 1 :

$$
\int_{S^{m}} \Omega_{h}^{m} d \xi=\int_{S^{m}} h \cdot d \xi=\int_{S^{m}} d \xi=1 .
$$

\section{The CONFORMAL INDEX AND THE GENERALIZEd POLYAKov FORMUla}

Return now to the setting of Section 1 , where $(M, g)$ is a Riemannian manifold of dimension $m$, possibly with spin structure (and thus a Clifford section $\gamma$ ). We would like to study the functional determinants of some natural differential operators $A$. For convenience, we separate the different kinds of assumptions needed for different parts of the theory. 
Analytic assumptions 4.1. Let $A$ be a differential operator of positive order on sections of a tensor-spinor bundle $\mathbb{V}$ over $M$. Suppose that $A$ is formally selfadjoint and has positive definite leading symbol $\sigma_{\text {lead }}(A)$; that is, $\sigma_{\text {lead }}(A)(x, \xi)$ is positive definite in End $\mathbb{V}_{x}$ for all $x \in M$ and $0 \neq \xi \in T_{x}^{*} M$.

Remark 4.2. The assumptions on the leading symbol make sense because tensorspinor bundles over a Riemannian manifold come equipped with Riemannian vector bundle structures. $A$ is, in particular, elliptic. Since $\sigma_{\text {lead }}(A)(x,-\xi)=$ $(-1)^{\text {ord } A} \sigma_{\text {lead }}(A)(x, \xi)$, the assumption of positive definite leading symbol forces the order of $A$ to be even. We shall fix the notation $2 \ell=2 \ell[A]>0$ for ord $A$.

Under the analytic assumptions 4.1 , we can write down the heat kernel trace expansion, and define the zeta function and functional determinant. Let $f$ denote an indeterminate element of $C^{\infty}(M)$; the heat expansion is

$$
\operatorname{Tr}_{L^{2}} f \exp (-t A) \sim \sum_{n=0}^{\infty} a_{n}(f, A) t^{(n-m) / 2 \ell}, \quad t \downarrow 0 .
$$

(See, e.g., [G4].) Here

$$
a_{n}(f, A)=\int_{M} f U_{n}[A] d v,
$$

where $U_{n}[A]$ is locally computable from the symbol of $A$ in local coordinates; $U_{n}[A]$ is polynomial in the total symbol, with coefficients that are smooth in the leading symbol. The invariants $U_{n}$ vanish for $n$ odd. The auxiliary function $f$ is a useful device for holding onto divergence terms in the heat coefficients; these disappear from view (by Stokes' Theorem) if we only consider $a_{n}[A]:=$ $a_{n}(1, A)=\int U_{n}[A]$. We could also keep track of the endomorphism-valued invariants in the heat kernel expansion by allowing $f$ to be valued in End $\mathbb{V}$, but this will not be useful for our purposes.

The heat operator trace can also be written in terms of the spectrum of $A$. Under the analytic assumptions $4.1, A$ has real eigenvalue spectrum $\lambda_{0} \leq \lambda_{1} \leq$ $\ldots \uparrow \infty . A$ may have finitely many negative and zero eigenvalues. The zeta function associated to $A$ is

$$
\zeta_{A}(s)=\sum_{\lambda_{j} \neq 0}\left|\lambda_{j}\right|^{-s} .
$$

$\zeta_{A}(s)$ is well-defined and holomorphic for large $\operatorname{Re} s$. Let $q[A]$ denote the multiplicity of 0 as an eigenvalue of $A$. Then by the heat expansion (4.1), (4.2)

$$
\begin{aligned}
\sum_{\lambda_{j} \neq 0} e^{-t\left|\lambda_{j}\right|} & =-q[A]+2 \sum_{\lambda_{j}<0} \sinh \left(t \lambda_{j}\right)+\sum_{n=0}^{N} a_{n}[A] t^{(n-m) / 2 \ell}+O\left(t^{(N-m+2) / 2 \ell}\right) \\
& =\sum_{n=0}^{N} \tilde{a}_{n}[A] t^{(n-m) / 2 \ell}+O\left(t^{(N-m+1) / 2 \ell}\right)
\end{aligned}
$$

where 


$$
\tilde{a}_{n}[A]=\left\{\begin{array}{l}
a_{n}[A]-q[A], \quad n=m, \\
a_{n}[A]+2 \sum_{\lambda_{j}<0} \lambda_{j}^{k} / k !, \quad n=m+2 \ell(1+2 k), \quad k \in \mathbb{N}, \\
a_{n}[A] \quad \text { otherwise. }
\end{array}\right.
$$

( $\tilde{a}_{n}[A]$ might not vanish for some odd $n$, but this can only happen if $m$ is odd.) Applying the Mellin transform, we get a meromorphic continuation of $\zeta_{A}(s)$ to $\mathbb{C}:$

$$
\begin{aligned}
\zeta_{A}(s)=\frac{1}{\Gamma(s)}\left(\sum_{n=0}^{N}\right. & \left(s-\frac{m-n}{2 \ell}\right)^{-1} \tilde{a}_{n}[A] \\
& \left.+\int_{0}^{1} t^{s-1} O\left(t^{(N-m+1) / 2 \ell}\right) d t+\int_{1}^{\infty} t^{s-1} \sum_{\lambda_{j} \neq 0} e^{-t\left|\lambda_{j}\right|} d t\right),
\end{aligned}
$$

where $O\left(t^{(N-m+1) / 2 \ell}\right)$ is the error term from (4.2). In particular, $\zeta_{A}(s)$ is regular at $s=0$, and we can define the functional determinant of $A$ by

$$
\operatorname{det} A:=(-1)^{\#\left\{\lambda_{j}<0\right\}} \exp \left(-\zeta_{A}^{\prime}(0)\right) \text {. }
$$

Before going further, we need to restrict to natural operators.

Naturality assumptions 4.3. $A$ is natural in the sense of Section 1, with coefficients that depend rationally on the dimension $m$. (In particular, $A$ might not exist for a finite set $\mathscr{C}_{A} \subset \mathbb{Z}^{+}$of dimensions.) With respect to uniform dilations of the metric, $A$ has homogeneity degree - ord $A$, in the sense that if $0<\alpha \in \mathbb{R}$ and $\bar{g}=\alpha^{2} g$, and (if spin structure is involved) $\bar{\gamma}=\alpha^{-1} \gamma$, then $\bar{A}=\alpha^{-2 \ell} A$. Furthermore, $A$ satisfies the analytic assumptions 4.1 categorically: the realization of $A$ on any Riemannian manifold $M$ for $m \notin \mathscr{C}_{A}$ satisfies 4.1.

Remark 4.4. By Weyl's invariant theory, the heat invariants $U_{n}$ of any $A$ satisfying the naturality assumptions 4.3 must be local scalar invariants in the sense of Section 1. Furthermore, under the above scaling, they must satisfy

$$
\bar{U}_{n}=\alpha^{-n} U_{n},
$$

by the heat expansion and the formula

$$
\exp \left(-t\left(\alpha^{-2 \ell} A\right)\right)=\exp \left(-\left(\alpha^{-2 \ell} t\right) A\right) \text {. }
$$

Recall from Section 1 that a local scalar invariant satisfying (4.3) has level $n$. The same terminology can be applied to natural scalar differential operators scaling as in (4.3). It is straightforward to show that we may measure the level via a "derivative count" as follows: If $U$ is a level $n$ monomial local invariant or monomial natural differential operator, of degree $\left(k_{R}, k_{\nabla}\right)$ in $(R, \nabla)$, then

$$
2 k_{R}+k_{\nabla}=n \text {. }
$$

(Occurrences of $g, g^{\sharp}, \gamma$ do not affect the level.) Our homogeneity assumption on $A$ just says that $A$ has a consistent level, equal to its order $2 \ell$. Thus $\sigma_{\text {lead }}(A)$ is polynomial in $g, g^{\sharp}, \gamma$; that is, no higher jets of these object are involved in the leading symbol. We also get that the section $\tilde{\sigma}_{\text {lead }}$ of $\left(\operatorname{Symm}\left(T^{*} M\right)^{\otimes 2 \ell}\right) \otimes$ End $\mathbb{V}$ representing the leading symbol is parallel (annihilated by $\nabla$ ), since $g, g^{\sharp}, \gamma$ are. 
Remark 4.5. It is important to note that the functional determinant is not invariant under uniform dilation (scaling) of the metric. Suppose, as above, that $\bar{g}=\alpha^{2} g$ for $0<\alpha \in \mathbb{R}$, and if applicable, $\bar{\gamma}=\alpha^{-1} \gamma$. Then

$$
\zeta_{\bar{A}}(0)=\zeta_{A}(0), \quad \operatorname{det} \bar{A}=\alpha^{-2 \ell \zeta_{A}(0)} \operatorname{det} A .
$$

That is, the quantity $\zeta_{A}(0)$ is scale-invariant, and the functional determinant has a scale homogeneity that depends on $\zeta_{A}(0)$. Thus the functional

$$
\mathscr{D}(A, g)=(\operatorname{vol} g)^{2 \ell \zeta_{A}(0) / m} \operatorname{det} A
$$

is a scale invariant "version" of the determinant. An added advantage of $\mathscr{D}(A, g)$ is that, like $\zeta_{A}(0)$ and the determinant, it is a spectral invariant, since $\ell[A]$ and vol $g$ are. ( $\operatorname{vol} g=c a_{0}[A]$, where $c$ is a nonzero constant depending only on $\sigma_{\text {lead }}[A]$.) We emphasize, however, that there is no reason to expect $\operatorname{det} A$ or $\mathscr{D}(A, g)$ to be the integral of a local expression, as is $a_{n}[A]$.

We shall now impose some additional conformal assumptions.

Conformal assumptions 4.6. $A$ is a positive integral power of a natural differential operator $D, A=D^{h}$, with $h$ independent of $m$, and $D$ conformally covariant in the sense of 1.2 , with a conformal bidegree $(a, b)=(a(m), b(m))$ that is rational in $m$.

Remark 4.7. We do not assume that $A$ itself is conformally covariant, or that $D$ has positive definite leading symbol. Working in this generality allows to handle, for example, the square $\nabla^{2}$ of the Dirac operator. Under 4.6 and the scale homogeneity assumptions of 4.3 , the conformal bidegree of $D$ is forced to take the form $(a, a+2 \ell / h)$.

An extremely important property from our point of view is a generalization of the scale-invariance property (4.4) to pointwise, or conformal scaling. This property is heavily dependent on the conformal assumptions 4.6. Following [BØ1], we call this a conformal index property.

Conformal Index Theorem 4.8 [B囚1]. Let $M$ be an m-dimensional compact manifold, and suppose that $A$ satisfies 4.1, 4.3, and 4.6, with $m \notin \mathscr{C}_{A}$. Then the quantities $q[A], \#\left\{\lambda_{j}<0\right\}, a_{m}[A]$, and $\zeta_{A}(0)$ are constant on each conformal class $\left[g_{0}\right]=\left\{e^{2 \omega} g_{0} \mid \omega \in C^{\infty}(M)\right\}$ of Riemannian metrics on $M$.

Proof. Since $\sigma_{\text {lead }}(A)=\sigma_{\text {lead }}(D)^{h}, D$ is elliptic, and thus has pure eigenvalue spectrum; as a result, $\mathscr{N}(A)=\mathscr{N}(D) . \quad q[A]=\operatorname{dim} \mathscr{N}(D)$ is conformally invariant because $\varphi \mapsto e^{a \omega} \varphi$ is a bijection of $\mathcal{N}\left(D_{0}\right)$ onto $\mathscr{N}\left(D_{\omega}\right)$. The conformal invariance of $\#\left\{\lambda_{j}<0\right\}$ follows from, e.g., [Bl, Proposition 1], using the just mentioned explicit knowledge of the motion of the 0 eigenspace under variation of $\omega$. Since $\zeta_{A}(0)=a_{m}[A]-q[A]$, we just need to show that $a_{m}[A]$ is conformally invariant.

For this, choose $\omega \in C^{\infty}(M)$, and consider the conformal curve of metrics $g_{\varepsilon \omega}=e^{2 \varepsilon \omega} g_{0}$. If we can show that the operator $\left.(d / d \varepsilon)\right|_{\varepsilon=0}$ annihilates the functional $a_{m}\left[A_{\varepsilon \omega}\right]$, we are done, since the result may then be applied with $g_{\varepsilon_{0} \omega}$ in place of $g_{0}$, and $\omega$ is arbitrary. The estimates of [Bס1] justify the following formal computation: 


$$
\begin{aligned}
\left.\left.\sum_{n=0}^{\infty}(d / d \varepsilon)\right|_{\varepsilon=0} a_{n}[A] t^{(n-m) / 2 \ell} \sim(d / d \varepsilon)\right|_{\varepsilon=0} \operatorname{Tr} \exp \left(-t A_{\varepsilon \omega}\right) \\
\quad=-t \operatorname{Tr}\left\{\left(\left.(d / d \varepsilon)\right|_{\varepsilon=0} A_{\varepsilon \omega}\right) \exp \left(-t A_{0}\right)\right\} \\
\quad=-t \operatorname{Tr}\left\{h D_{0}^{h-1}\left(\left.(d / d \varepsilon)\right|_{\varepsilon=0} D_{\varepsilon \omega}\right) \exp \left(-t A_{0}\right)\right\} \\
=-t \operatorname{Tr}\left\{h D_{0}^{h-1}\left(-(2 \ell / h) \omega D_{0}+a\left[D_{0}, \mu(\omega)\right]\right) \exp \left(-t A_{0}\right)\right\} \\
=2 t \ell \operatorname{Tr} \omega\left\{D_{0}^{h} \exp \left(-t A_{0}\right)\right\} \\
=2 t \ell \operatorname{Tr} \omega\left\{A_{0} \exp \left(-t A_{0}\right)\right\} \\
=-2 \ell t(d / d t) \operatorname{Tr} \omega \exp \left(-t A_{0}\right) \\
\sim \sum_{n=0}^{\infty}(m-n) a_{n}\left(\omega, A_{0}\right) t^{(n-m) / 2 \ell} \\
\sim \sum_{n=0}^{\infty}(m-n) t^{(n-m) / 2 \ell} \int_{M} \omega\left(U_{n}[A] d v\right)_{0},
\end{aligned}
$$

where, of course, the asymptotics are for $t \downarrow 0$. Here we have used the fact that functions of $D$ and $A$ commute, and that $e^{-t A_{0}}$ is a smoothing operator for $t>0$. Comparing coefficients for $n=m$, we get the desired result.

As a corollary to the proof, we get a formula that will be useful below:

Corollary 4.9 [BØ2]. Under the assumptions of Theorem 4.8,

$$
\left.(d / d \varepsilon)\right|_{\varepsilon=0} a_{n}\left[A_{\varepsilon \omega}\right]=(m-n) \int_{M} \omega\left(U_{n}[A] d v\right)_{0} .
$$

Thus the functional $(m-n)^{-1} a_{n}\left[A_{\omega}\right]$ is a conformal primitive for $U_{n}[A]$ if $n \neq m$.

If we wish, we can use the corollary to "reconstruct" the divergence terms in $U_{n}[A]$ from the integrated invariant $a_{n}[A]$, as long as $n \neq m$. In fact, the rational dependence on dimension assumed in 4.3 allows us to analytically continue in $m$ (with some additional assumptions on $\sigma_{\text {lead }}(A)$; see 5.1 (a) below), so the restriction $n \neq m$ will not really be a problem.

We shall now show that the functional determinant is the "missing" conformal primitive in the above, i.e., that of $U_{m}[A]$. This will complete a very strong analogy between the local invariants $Q_{n}$ and the quadratic form $\left(P_{m} \omega, \omega\right)_{L^{2}}$ studied in Sec. 1 on one hand; and the local invariants $U_{n}[A]$ and the functional determinant on the other. This analogy will have more than esthetic value to us; it will actually lead to an efficient procedure for computing quotients of the form $\left(\operatorname{det} A_{\omega}\right) /\left(\operatorname{det} A_{0}\right)$.

Theorem 4.10 (Generalized Polyakov formula [BØ2]). Suppose A satisfies 4.1, 4.3, and 4.6. Let $\left(M, g_{0}\right)$ be a particular $m$-dimensional manifold, $m \notin \mathscr{C}_{A}$, on which $\mathscr{N}(A)=0$. Then

$$
\left.(d / d \varepsilon)\right|_{\varepsilon=0} \zeta_{A_{\varepsilon \omega}}^{\prime}(0)=2 \ell a_{m}\left(\omega, A_{0}\right) .
$$

Proof. First assume that $\#\left\{\lambda_{j}<0\right\}=0$, so that each $A_{\omega}$ is a positive operator. The estimates in [BØ 1, BØ2] allow us to conclude that $\left.(d / d \varepsilon)\right|_{\varepsilon=0} \zeta_{A_{\varepsilon \omega}}(s)$ is 
meromorphic in $s$, and that we can interchange the order of conformal variation and analytic continuation, or of conformal variation and $s$-differentiation. (Besides uniform estimates, this depends on the conformal invariance of $q[A]$ and of $\#\left\{\lambda_{j}<0\right\}$, because of the way the nonpositive spectrum was handled in the definition of the functional determinant.) For $\operatorname{Re} s$ large,

$\left.(d / d \varepsilon)\right|_{\varepsilon=0} \zeta_{A_{\varepsilon \omega}}^{\prime}(s)=\left.(d / d s)(d / d \varepsilon)\right|_{\varepsilon=0} \zeta_{A_{\varepsilon \omega}}(s)$

$$
\begin{aligned}
& =(d / d s)\left\{\frac{1}{\Gamma(s)} \int_{0}^{\infty} t^{s-1}\left\{\left.(d / d \varepsilon)\right|_{\varepsilon=0} \operatorname{Tr} \exp \left(-t A_{\varepsilon \omega}\right)\right\} d t\right\} \\
& =-(d / d s)\left\{\frac{2 \ell}{\Gamma(s)} \int_{0}^{\infty} t^{s}(d / d t) \operatorname{Tr}\left(\omega \exp \left(-t A_{0}\right)\right) d t\right\} \\
& =(d / d s)\left\{\frac{2 \ell s}{\Gamma(s)} \int_{0}^{\infty} t^{s-1} \operatorname{Tr}\left(\omega \exp \left(-t A_{0}\right)\right) d t\right\} .
\end{aligned}
$$

Analytically continuing this formula, the value at $s=0$ is the same as that of

$$
\frac{2 \ell}{\Gamma(s)} \int_{0}^{\infty} t^{s-1} \operatorname{Tr}\left(\omega \exp \left(-t A_{0}\right)\right) d t
$$

viz., $2 \ell a_{m}\left(\omega, A_{0}\right)$.

To dispense with the positivity assumption on $A$, note that we have proved the result for the positive operator $A^{2}$. But $\zeta_{A^{2}}(s)=\zeta_{A}(2 s)$, so $\zeta_{A^{2}}^{\prime}(0)=$ $2 \zeta_{A}^{\prime}(0) ;$ and by [FG], $U_{m}\left[A^{2}\right]=U_{m}[A]$.

Remark 4.11. The effect of zero eigensections on the above formula and argument is as follows. Since $q[A]$ is conformally invariant, formula (4.5) is still correct. Formula (4.6) is also correct, and can be rewritten as

$$
-(d / d s)\left\{\frac{2 \ell}{\Gamma(s)} \int_{0}^{\infty} t^{s}(d / d t) \operatorname{Tr}\left(\omega\left(\exp \left(-t A_{0}\right)-\operatorname{Proj}_{\mathscr{N}\left(A_{0}\right)}\right)\right) d t\right\}
$$

It is now clear that the integral converges, and that the $\int_{1}^{\infty}$ part does not affect the value of the expression at $s=0$. The kernel function of

$$
\omega\left\{\exp \left(-t A_{0}\right)-\operatorname{Proj}_{\mathscr{N}\left(A_{0}\right)}\right\}
$$

is

$$
\omega(x)\left\{H(t, x, y)-\sum_{\lambda_{j}=0} \varphi_{j}(x) \otimes \varphi_{j}^{*}(y)\right\},
$$

where $H(t, x, y)$ is the kernel function of $\exp \left(-t A_{0}\right)$, and $\left\{\varphi_{j}\right\}$ is an $L^{2}$ orthonormal basis of the zero eigensections. The conclusion is that

$$
\begin{aligned}
\left.(d / d \varepsilon)\right|_{\varepsilon=0} \zeta_{A_{\varepsilon \omega}}^{\prime}(0) & =2 \ell\left(a_{m}\left(\omega, A_{0}\right)-\int_{M} \omega \sum_{\lambda_{j}=0}\left|\varphi_{j}(x)\right|^{2}(d v)_{0}\right) \\
& =2 \ell \int_{M} \omega\left(U_{m}[A]-\sum_{\lambda_{j}=0}\left|\varphi_{j}(x)\right|^{2}\right)(d v)_{0}
\end{aligned}
$$

Thus we can compute with the generalized Polyakov formula if $\mathscr{N}\left(A_{0}\right)=0$, or if we have explicit knowledge of $\mathscr{N}\left(A_{0}\right)$. For example, if $\left(M, g_{0}\right)$ is a flat torus, any parallel section is annihilated by any natural differential operator, so 
zero eigenvalues are unavoidable. On the other hand, $\mathscr{N}\left(A_{0}\right)$ consists exactly of the parallel sections in this case, i.e., of the sections that have constant components in the standard trivialization; thus we know these sections explicitly. The conformal covariance relation then gives us knowledge of the null space in conformally related metrics, i.e., of $\mathscr{N}\left(A_{\omega}\right)$.

Remark 4.12. Because $U_{m}[A]$ vanishes for $m$ odd, the functional determinant of an operator satisfying our conformal assumptions is conformally rigid in odd dimensions in the absence of a null space. If there is a null space, the conformal variation of the determinant depends only on the conformal variation of this null space. For boundary value problems, however, we do get an interesting functiorial in odd dimensions, since there is a nonvanishing boundary contribution to $a_{\text {odd }}(\omega, A)$ [BG1, BG2].

We would now like to compute the quotients $\left(\operatorname{det} A_{\omega}\right) /\left(\operatorname{det} A_{0}\right)$; what we shall actually write down is the equivalent information

$$
-\log \left(\left(\operatorname{det} A_{\omega}\right) /\left(\operatorname{det} A_{0}\right)\right)=-\log \left|\operatorname{det} A_{\omega}\right|+\log \left|\operatorname{det} A_{0}\right|,
$$

or the equivalent scale invariant information

$$
-\log \left(\mathscr{D}\left(A, e^{2 \omega} g_{0}\right) / \mathscr{D}\left(A, g_{0}\right)\right)=-\frac{2 \ell \zeta_{A}(0)}{m} \log \frac{\int_{M} e^{m \omega}(d v)_{0}}{\operatorname{vol}\left(g_{0}\right)}-\log \frac{\operatorname{det} A_{\omega}}{\operatorname{det} A_{0}} .
$$

(Note that sgn det $A_{\omega}=\operatorname{sgn} \operatorname{det} A_{0}$ since $\#\left\{\lambda_{j}<0\right\}$ is conformally invariant, and that the scale invariant quantity makes sense as a functional on the conformal class $\left[g_{0}\right]$ because $\zeta_{A}(0)$ is conformally invariant.) According to the generalized Polyakov formula, say for $\mathscr{N}(A)=0$, we just need to compute a conformal primitive for $U_{m}[A]$ that vanishes at $\omega=0$ in order to compute (4.7).

\section{ThE FUNCTIONAL DETERMINANT IN DIMENSIONS 2 AND 4}

To avoid having to compute the divergence terms in $U_{m}[A]$, replacing this information by the knowledge of $a_{m}[A]$ for each even dimension $m \notin \mathscr{C}_{A}$, we need the following technical assumption on $\left(m, U_{m}[A]\right)$. The assumption is satisfied in all situations where we are able to compute $U_{m}[A]$, so it is no impediment to our calculations. One might conjecture that it is true quite generally.

Technical assumptions 5.1. Fix $n \in 2 \mathbb{Z}^{+}$, and make the following assumption on $(n, A)$ :

(a) There exists a universal function $u_{A}(m)$, meromorphic on $\mathbb{C}$ and depending only on the formal polynomial expression of $\sigma_{\text {lead }}(A)$, such that the coeffcients in the formal polynomial expression for $u_{A}(m) U_{n}[A]$ are rational in $m$ for $m \in 2 \mathbb{Z}^{+}, m \notin \mathscr{C}_{A}$.

(b) There is a rational function $\alpha_{n}(m)$ and a local scalar $\mathrm{O}(m)$ invariant $B_{n}(m)$ depending rationally on $m \in 2 \mathbb{Z}^{+}$, both regular at $m=n$, such that

$$
u_{A}(m) U_{n}[A] \simeq \alpha_{n}(m) Q_{n}+(m-n) B_{n}(m) \text { modulo } \mathscr{R}(\delta),
$$

for all conformally flat compact Riemannian manifolds of even dimension $m \notin$ $\mathscr{C}_{A}$. Here $\delta$ is the formal adjoint of the exterior derivative $d$ on functions, and $\mathscr{R}(\delta)$ is its range.

Remark 5.2. Local scalar $\mathrm{O}(m)$ invariants with dimension-dependent coefficients can be viewed in the light of Gilkey's Theorem [G1, BFG, G4]. Among 
other things, Gilkey's Theorem states that as $m$ increases, the space $L_{n}^{m}$ of level $n$ local scalar $\mathrm{O}(m)$ invariants on $m$-dimensional Riemannian manifolds stabilizes at $m=n$ :

$$
L_{n}^{m} \cong L_{n}^{n}, \quad m \geq n .
$$

The isomorphisms are natural, and obtained by stabilization maps $s_{n}^{m}: L_{n}^{m+1} \rightarrow$ $L_{n}^{m} . \quad s_{n}^{m}$ evaluates on a product manifold $N \times S^{1}, \operatorname{dim} N=m$, and then restricts to the submanifold $N \times\{1\} \simeq N$. We denote by $L_{n}$ the direct limit of the $L_{n}^{m}$ as $m \uparrow \infty$; this is naturally isomorphic to $L_{n}^{n}$. Let

$$
\mathscr{L}_{n}=\operatorname{Rat}(m) \otimes_{\mathbb{R}} L_{n},
$$

where Rat $(m)$ is the field of rational functions in $m$. We shall need to consider local invariants in the conformally flat category. If $(M, g)$ is conformally flat, then $M$ is covered by charts $\left(X, g_{b}\right)$, where $g_{b}$ is the standard flat metric on $X$ and $g=e^{2 h_{X}} g_{\mathrm{b}}$. By [Bra1, Section 1], each local invariant $\varphi \in L_{n}^{m}$ has the form

$$
\varphi_{g}=e^{-n h_{X}} \tilde{\varphi}\left(\nabla h_{X}, \ldots, \nabla^{n} h_{X}\right),
$$

when evaluated in the metric $g$, where $\tilde{\varphi}$ is a universal (independent of the choice of $\left.\left(X, g_{b}\right)\right)$ polynomial, homogeneous of degree $n$ in the $g_{b}$ covariant derivative $\nabla$. Entirely similar statements can be made about local tensorial and operator valued invariants. By [BGP, Theorem 0.1], all universal scalar and differential form expressions (5.1) are restrictions of local invariants from $L_{n}^{m}$, or the similarly defined space $L_{n, p}^{m}$ of $p$-form invariants, in the conformally flat category; we denote by $\tilde{L}_{n}^{m}$ and $\tilde{L}_{n, p}^{m}$ the spaces of such restrictions. It will also be technically useful to consider the space of formal expressions (5.1) which are not necessarily universal; we shall call this $\hat{L}_{n}^{m}$, or, in the $p$-form case, $\hat{L}_{n, p}^{m}$. To see the difference between the $\hat{L}$ and $\tilde{L}$ spaces in an example, note that $\tilde{L}_{2}^{m}$ has dimension 1 , and is generated by the expression for the scalar curvature $(m \geq 2)$; but $\hat{L}_{2}^{m}$ has dimension 2 (and basis $e^{-2 h_{X}} \Delta h_{X}, e^{-2 h_{X}}\left|\nabla h_{X}\right|^{2}$ ). The $\hat{L}$ spaces are useful because they have bases which are $m$-stable for $m \geq n$, consisting of monomials

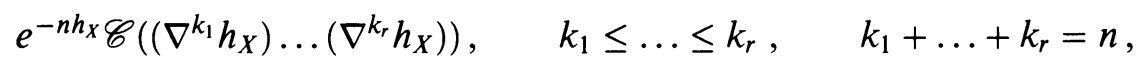

where $\mathscr{C}$ is some contraction and permutation of arguments operator which produces a form of the right degree. By the proof of [BGP, Theorem 0.1], the space of invariant scalar expressions (5.1) with rational-in- $m$ coefficients is exactly the space $\tilde{\mathscr{L}}_{n}$ of restrictions of elements of $\mathscr{L}_{n}$ to the conformally flat category. When we analytically continue in dimension in the conformally flat category, the process will implicitly be an analytic continuation of expressions (5.1). In any given explicit calculation, one needs to watch whether the $m$ in question is a pole of any of the coefficients involved.

Remark 5.3. Suppose $A$ satisfies the naturality assumptions 4.3 , acting on sections of a tensor-spinor bundle $\mathbb{V}$, that ord $A=2$ and $\sigma_{2}(A)$ is scalar, i.e., $\sigma_{2}(A)(x, \xi)=|\xi|^{2} \mathrm{Id}_{\mathbf{v}_{x}}$. Then examination of the algorithm that produces the heat invariants [G4] shows that the coefficients appearing in the formal polynomial expression for $(4 \pi)^{m / 2}(\operatorname{dim} \mathbb{V})^{-1} U_{n}[A]$ are rational in $m$, so we have no rationality problems so long as $(4 \pi)^{m / 2}(\operatorname{dim} \mathbb{V})^{-1}$ is meromorphic. This is one 
reason for restricting to even $m$ in 5.1: the fiber dimension of the spinor bundle is $2^{[m / 2]}$; this is the holomorphic function $2^{m / 2}$ when applied to even $m$, but a different holomorphic function, $2^{(m-1) / 2}$, when applied to odd $m$. The point is that we have to keep track of the dependence of the fiber dimension $\operatorname{dim} \mathbb{V}$ on $m$, and that for the operators $Y$ and $\nabla^{2}$, this dependence causes no problem. If $\operatorname{ord} A=4$ and $\sigma_{4}(A)$ is scalar in the sense that $\sigma_{4}(A)(x, \xi)=|\xi|^{4} \operatorname{Id}_{\mathbf{v}_{x}}$, the same can be said with a certain meromorphic function in place of $(4 \pi)^{\mathrm{m} / 2}$; unlike the second order case, this function now depends on $n$ [G3, FG]. Eventually, we need to apply meromorphic continuation to coefficients in formal polynomials defining local invariants; the limit point involved is $m=\infty$, so we need meromorphicity there. This is the reason for extracting the universal meromorphic factor in 5.1: so that we shall really only need to apply meromorphic continuation to rational functions (whose singularities at infinity, if any, are poles). The assumptions are certainly not the weakest under which meromorphic continuation can be performed, but suffice for the present purposes.

Remark 5.4. Note that there are "new" identities in the conformally flat category which do not involve the Weyl tensor $C$ directly. The most basic example follows from the Bianchi identity: in an orthonormal frame, summing over repeated indices,

$$
C_{i j k l \mid i}=(m-3)\left(V_{j l \mid k}-V_{j k \mid l}\right) .
$$

Thus for a conformally flat metric in dimension $m \geq 4$, the tensor $\nabla V$ is symmetric in all three of its arguments. (This property also holds in dimension 3, and in fact characterizes conformal flatness there.) For general non-conformally flat metrics however, this identity (the one that states that $\nabla V$ is completely symmetric) does not hold.

Remark 5.5. Some subspaces of the spaces introduced in Remark 5.2 will be important to us. Let $\tilde{\mathscr{L}}_{n-1,1}$ be the space of invariant level $n-1$ one-form expressions $(5.1)$ with rational-in- $m$ coefficients. The divergence $\delta$ carrying one-forms to functions determines spaces

$$
\delta L_{n-1,1}^{m} \subset L_{n}^{m}, \quad \delta \tilde{L}_{n-1,1}^{m} \subset \tilde{L}_{n}^{m}, \quad \delta \tilde{\mathscr{L}}_{n-1,1} \subset \mathscr{L}_{n} .
$$

of exact divergences. In the (5.1) picture, the fact that $\delta$ is conformally covariant of bidegree $(m-2, m)$ gives

$$
\delta_{g} \varphi_{g}=e^{-(n+2) h_{X}}\left\{\delta_{b} \tilde{\varphi}-(m-2-n) l\left(d h_{X}\right) \tilde{f}\right\},
$$

where $l$ is interior multiplication. Another class of interest to us is that of level $n$ local invariants $U$ satisfying

$$
\left.(d / d \varepsilon)\right|_{\varepsilon=0} \int_{M}(U d v)_{\varepsilon \omega}=(m-n) \int_{M} \omega(U d v)_{0}
$$

under variation along the conformal curve $g_{\varepsilon \omega}=e^{2 \varepsilon \omega} g_{0}$; this condition makes sense within $L_{n}^{m}, \mathscr{L}_{n}, \tilde{L}_{n}^{m}$, or $\tilde{\mathscr{L}}_{n}$, giving rise to classes $C L_{n}^{m}, C \mathscr{L}_{n}, C \tilde{L}_{n}^{m}$, and $C \tilde{\mathscr{L}}_{n}$ of relative conformal invariants. In the conformally flat case, $Q_{n}$ satisfies (5.2) by Corollary 1.4 ; by Corollary 4.9 , so does $U_{n}[A]$ for $A$ satisfying $4.1,4.3$, and 4.6 (and for $U_{n}[A]$, conformal flatness need not be assumed). $C L_{m}^{m}$ and $C \tilde{L}_{m}^{m}$ consist of local invariants whose integrals are conformal invariants; and furthermore, $\delta L_{m-1,1}^{m} \subset C L_{m}^{m}, \delta \tilde{L}_{m-1,1}^{m} \subset C \tilde{L}_{m}^{m}$, and 
$\delta \tilde{\mathscr{L}}_{n-1,1} \subset C \tilde{\mathscr{L}}_{n}$. [BGP, Theorem 0.2] shows that $C \tilde{L}_{m}^{m} / \delta \tilde{L}_{m-1,1}^{m}$ is onedimensional, and is generated by the class of the Pfaffian (Euler polynomial) $\mathrm{Pff}_{m}$. We know from Theorem 2.8(f) that the class of $Q_{m}$ is nonzero, so $Q_{m}$ is also a generator for $C \tilde{L}_{m}^{m} / \delta \tilde{L}_{m-1,1}^{m}$. Thus we need assumption 5.1(b) just to guarantee the rationality of $\alpha_{n}(m)$ and $B_{n}(m)$.

We are now ready to write a very general formula for the functional determinant in the conformally flat case.

Theorem 5.6. Under the assumptions 4.1, 4.3, and 4.6 on $A$, and 5.1 on $(m, A)$, with $m \in 2 \mathbb{Z}^{+}$and $m \notin \mathscr{C}_{A}$, the quantity $\mathscr{A}$ defined by

$$
\begin{aligned}
u_{A}(m) \mathscr{A}:= & \frac{1}{2} \alpha_{m}(m) \int \omega\left\{\left(Q_{m} d v\right)_{\omega}+\left(Q_{m} d v\right)_{0}\right\} \\
& +\int\left\{\left(B_{m}(m) d v\right)_{\omega}-\left(B_{m}(m) d v\right)_{0}\right\} \\
= & \alpha_{m}(m)\left\{\frac{1}{2} \int \omega\left(\left(P_{m}\right)_{0} \omega\right)(d v)_{0}+\int \omega\left(Q_{m} d v\right)_{0}\right\} \\
& +\int\left\{\left(B_{m}(m) d v\right)_{\omega}-\left(B_{m}(m) d v\right)_{0}\right\}
\end{aligned}
$$

is a conformal primitive for $U_{m}[A]$ on conformally flat conformal classes $\left[g_{0}\right]$, and vanishes at $\omega=0$. As a result, given a particular $m$-dimensional conformally flat compact Riemannian manifold $\left(M, g_{0}\right)$ on which $\mathcal{N}(A)=0$ (and which has spin structure if required to define $A)$,

$$
2 \ell \mathscr{A}=-\log \frac{\operatorname{det} A_{\omega}}{\operatorname{det} A_{0}}=-\log \frac{\mathscr{D}\left(A, e^{2 \omega} g_{0}\right)}{\mathscr{D}\left(A, g_{0}\right)}+\frac{2 \ell \zeta_{A}(0)}{m} \log \frac{\int_{M} e^{m \omega}(d v)_{0}}{\operatorname{vol}\left(g_{0}\right)}
$$

Proof. Let

$$
V_{n}[A]:=u_{A}(m) U_{n}[A]-\alpha_{n}(m) Q_{n}
$$

then

$$
\int V_{n} d v=(m-n) \int B_{n}(m) d v
$$

for $m \notin \mathscr{C}_{A}$. Since $V_{n}[A] \in C \tilde{L}_{n}^{m}$,

$$
\begin{aligned}
\left.(m-n)(d / d \varepsilon)\right|_{\varepsilon=0} & \int\left(B_{n}(m) d v\right)_{\varepsilon \omega}=\left.(d / d \varepsilon)\right|_{\varepsilon=0} \int\left(V_{n} d v\right)_{\varepsilon \omega} \\
& =(m-n) \int \omega\left(V_{n} d v\right)_{0} .
\end{aligned}
$$

Thus $\int B_{m}(m) d v$ is a conformal primitive for $V_{m}$ in dimension $m$, and $\int\left\{\left(B_{m}(m) d v\right)_{\omega}-\left(B_{m}(m) d v\right)_{0}\right\}$ is a conformal primitive that vanishes at $\omega=0$. By Corollary 1.7, $\frac{1}{2} \int \omega\left\{\left(Q_{m} d v\right)_{\omega}+\left(Q_{m} d v\right)_{0}\right\}$ is a conformal primitive for $Q_{m}$ vanishing at $\omega=0$. The determinant formulas now follow from Theorem 4.10.

The functional

$$
\int\left\{\left(B_{m}(m) d v\right)_{\omega}-\left(B_{m}(m) d v\right)_{0}\right\}
$$

is invariant under change of scale ( $\omega \mapsto \omega+$ const ), so the "correction" term in (5.3) is a correction to the functional involving $Q_{m}$ (or $Q_{m}$ and $P_{m}$ ). Indeed, 
the coefficients of the correction term and the $Q_{m}$ term are related by

$$
\zeta_{A}(0)+q[A]=a_{m}[A]=u_{A}(m)^{-1} \alpha_{m}(m) \int\left(Q_{m} d v\right)_{0} .
$$

This allows us to write the scale invariant determinant functional in a way which is well adapted to estimation techniques:

Corollary 5.7. Under the assumptions of Theorem 5.6 (including $\mathscr{N}(A)=0$ ), if $Q_{m}$ is constant on $\left(M, g_{0}\right)$,

$$
\begin{aligned}
-(2 \ell)^{-1} u_{A}(m) & \log \frac{\mathscr{D}\left(A, e^{2 \omega} g_{0}\right)}{\mathscr{D}\left(A, g_{0}\right)} \\
= & \alpha_{m}(m)\left\{-\frac{\left(Q_{m}\right)_{0}}{m} \log \frac{\int_{M} e^{m(\omega-\omega)}(d v)_{0}}{\operatorname{vol}\left(g_{0}\right)}+\frac{1}{2} \int_{M} \omega\left(\left(P_{m}\right)_{0} \omega\right)(d v)_{0}\right\} \\
& +\int\left\{\left(B_{m}(m) d v\right)_{\omega}-\left(B_{m}(m) d v\right)_{0}\right\},
\end{aligned}
$$

where $\bar{\omega}$ is the $(d v)_{0}$-average of $\omega$.

Note that the term involving $P_{m}$ is scale invariant because $P_{m}$ annihilates constants in dimension $m$.

We shall illustrate the theory by computing the functional determinants of the conformal Laplacian $Y$, and of the square $\not^{2}$ of the Dirac operator on conformally flat manifolds in dimensions 2,4 , and 6 . Since both operators have second order scalar leading symbol, we may take $u_{A}(m)=(4 \pi)^{m / 2}$ in both cases (Remark 5.3). The two-dimensional theory has been much studied; see, e.g., [On, OPS1-2]. The four-dimensional theory was developed in [BØ3, BCY], and the six-dimensional theory is presented for the first time here. The Dirac operator is conformally covariant,

$$
\begin{aligned}
& g_{\omega}=e^{2 \omega} g_{0}, \gamma_{\omega}=e^{-\omega} \gamma_{0}, \\
& \qquad \omega \in C^{\infty}(M) \Rightarrow \nabla_{\omega}=e^{-(m+1) \omega / 2} \nabla_{0} \mu\left(e^{(m-1) \omega / 2}\right),
\end{aligned}
$$

so the conformal assumption 4.6 is satisfied in both cases. Our formulas for the heat invariants come from [G2]. These formulas can be applied in particular to natural operators of the form $\nabla^{*} \nabla-\mathscr{E}$ on tensor-spinor bundles $\mathbb{V}$, where $\mathscr{E}$ is a smooth section of End $\mathbb{V}$. For $Y$, this is $\mathscr{E}_{Y}=-(m-2) J / 2$, while for $\not \nabla^{2}$, this is $\mathscr{E}_{\not \nabla^{2}}=-\tau \mathrm{Id}_{\Sigma} / 4=-(m-1) J \mathrm{Id}_{\Sigma} / 2$ by the Lichnerowicz formula [Lic]. To evaluate $a_{4}\left[\not^{2}\right]$ and $a_{6}\left[\not^{2}\right]$, we shall need the spin curvature [Ko],

$$
W_{i j}=-\frac{1}{4} R_{k l i j} \gamma_{k} \gamma_{l}
$$

in an orthonormal frame.

In studying two-dimensional manifolds, we are especially interested in local scalar invariants of level 2 . The space $\mathscr{L}_{2}$ of such is one-dimensional, and is generated by the scalar curvature $\tau$. We could (and shall) also use our normalized scalar curvature $J$ as the generator of $\mathscr{L}_{2}$. All two-dimensional manifolds are conformally flat, so our theory is completely general thus far. By, e.g., [G2],

$$
6(4 \pi)^{m / 2} N^{-1} a_{2}\left[\nabla^{*} \nabla-\mathscr{E}\right]=\int\{\tau+6 \mathscr{E}\} d v,
$$

where $N$ is the fiber dimension of the bundle $\mathbb{V}$ on whose sections $A$ acts. 
Thus

$$
\begin{aligned}
6(4 \pi)^{m / 2} a_{2}[Y] & =(-m+4) \int J d v, \\
6(4 \pi)^{m / 2} 2^{-[m / 2]} a_{2}\left[\nabla^{2}\right] & =(-m+1) \int J d v .
\end{aligned}
$$

(Though we have written the spinor formula for arbitrary $m$, our rationality assumptions are satisfied only when we restrict to even $m$, and it is only this part of the formula that we actually use.) Since $J=Q_{2}, Y=P_{2}$, and $Y$ is just $\Delta$ in dimension $m=2, J$ has the conformal primitive

$$
\frac{1}{2} \int\left\{(J d v)_{\omega}+(J d v)_{0}\right\}=\frac{1}{2} \int \omega\left(\Delta_{0} \omega\right)(d v)_{0}+\int \omega(J d v)_{0}
$$

in dimension 2. $\Delta$ always has a one-dimensional null space generated by the constant function; in the metric $g$, the constant $\operatorname{vol}(g)^{-1 / 2}$ is an $L^{2}$ orthonormal basis of $\mathscr{N}(\Delta)$. By Remark 4.11, the quotient of functional determinants has an extra term coming from the conformal primitive of

$$
\frac{\int \omega(d v)_{\omega}}{\int(d v)_{\omega}}
$$

that primitive being $\left(\log \int(d v)_{\omega}\right) / 2$. Note that $\operatorname{Pff}_{2}=\tau / 4 \pi=J / 2 \pi$, so the conformal index of $\Delta$ is

$$
\zeta_{\Delta}(0)=a_{2}[\Delta]-1=\frac{1}{6} \chi(M)-1
$$

by (5.4), where $\chi(M)$ is the Euler characteristic. The upshot is

Theorem 5.8. On a compact manifold $M$ of dimension $m=2$, in any Riemannian conformal class $\left[g_{0}\right]$,

$$
\begin{aligned}
-\log \frac{\operatorname{det} \Delta_{\omega}}{\operatorname{det} \Delta_{0}} & =-\log \frac{\int_{M} e^{2 \omega}(d v)_{0}}{\operatorname{vol}\left(g_{0}\right)}+\frac{1}{12 \pi} \int_{M} \omega\left\{(J d v)_{\omega}+(J d v)_{0}\right\} \\
& =-\log \frac{\int_{M} e^{2 \omega}(d v)_{0}}{\operatorname{vol}\left(g_{0}\right)}+\frac{1}{12 \pi} \int_{M} \omega\left(\Delta_{0} \omega\right)(d v)_{0}+\frac{1}{6 \pi} \int_{M} \omega(J d v)_{0} .
\end{aligned}
$$

The scale invariant determinant functional is

$$
\begin{gathered}
-\log \frac{\mathscr{D}\left(\Delta, e^{2 \omega} g_{0}\right)}{\mathscr{D}\left(\Delta, g_{0}\right)}=-\frac{1}{6} \chi(M) \log \frac{\int_{M} e^{2 \omega}(d v)_{0}}{\operatorname{vol}\left(g_{0}\right)}+\frac{1}{12 \pi} \int_{M} \omega\left\{(J d v)_{\omega}+(J d v)_{0}\right\} \\
=-\frac{1}{6} \chi(M) \log \frac{\int_{M} e^{2 \omega}(d v)_{0}}{\operatorname{vol}\left(g_{0}\right)}+\frac{1}{12 \pi} \int_{M} \omega\left(\Delta_{0} \omega\right)(d v)_{0}+\frac{1}{6 \pi} \int_{M} \omega(J d v)_{0} .
\end{gathered}
$$

In particular, if $J_{0}$ is constant,

$$
-\log \frac{\mathscr{D}\left(\Delta, e^{2 \omega} g_{0}\right)}{\mathscr{D}\left(\Delta, g_{0}\right)}=-\frac{1}{6} \chi(M) \log \frac{\int_{M} e^{2(\omega-\omega)}(d v)_{0}}{\operatorname{vol}\left(g_{0}\right)}+\frac{1}{12 \pi} \int_{M} \omega\left(\Delta_{0} \omega\right)(d v)_{0},
$$

where $\bar{\omega}$ is the $(d v)_{0}$-average of $\omega$. If $\left(M, g_{0}\right)$ is $S^{2}$ with its standard metric $g_{0}$, and $d \xi=(d v)_{0} / 4 \pi,(d \xi)_{\omega}=(d v)_{\omega} / 4 \pi$ are the normalized measures of Section 3, then

$$
-\log \frac{\mathscr{D}\left(\Delta, e^{2 \omega} g_{0}\right)}{\mathscr{D}\left(\Delta, g_{0}\right)}=\frac{1}{3}\left(-\log \int_{S^{2}} e^{2(\omega-\bar{\omega})} d \xi+\int_{S^{2}} \omega\left(\Delta_{0} \omega\right) d \xi\right) .
$$


Note that by the Uniformization Theorem, we are entitled to assume that $J_{0}$, the Gauss curvature of the background metric $g_{0}$, is constant. Remarkably, the quantity in (5.6) is exactly that estimated by the Moser-Trudinger inequality, i.e., the $m=2$ case of Theorem 3.7. This leads directly to Onofri's result on the maximum value of the functional determinant.

Corollary 5.9 [On]. Among all volume $4 \pi$ metrics $g$ on $S^{2}$, $\operatorname{det} \Delta_{g}$ is maximized exactly at those diffeomorphic to the standard metric $g_{0}$; i.e., for which $g=\varphi^{*} g_{0}, \varphi \in \operatorname{Diffeo}\left(S^{2}\right)$.

Here we have used the fact that $\Delta$ has no negative eigenvalues to reduce to the extremal problem for the quantity (5.6). The fact that one can maximize over the space of all metrics is special to dimension 2: given any Riemannian metric $g$ on $S^{2}$, there is a $\varphi \in \operatorname{Diffeo}\left(S^{2}\right)$ with $\varphi^{*} g \in\left[g_{0}\right]$ [OPS1]. Since the determinant and the scale-invariant determinant functional are spectral invariants, they are diffeomorphism invariants, and this reduces us to the conformal problem of the Moser inequality. For general compact 2-manifolds $M$, roughly speaking, there are only "finitely many degrees of freedom" remaining once conformal change and diffeomorphism are "drained out" [OPS1]. In its precise form, this is a statement about the reduced space of metrics $\mathscr{G} / \operatorname{Diffeo}(M) \ltimes C_{+}^{\infty}(M)$, where $\mathscr{G}$ is the space of Riemannian metrics and $C_{+}^{\infty}(M)$ acts by conformal change of metric.

If $\mathscr{N}(\not \nabla)=0$, the conformal index of $\not^{2}$ is

$$
\zeta_{\nabla^{2}}(0)=-\chi(M) / 12
$$

by $(5.5)$, so we have:

Theorem 5.10. On a compact spin manifold of dimension $m=2$, in any Riemannian conformal class $\left[g_{0}\right]$ where the conformally invariant condition $\mathcal{N}(\mathbb{\nabla})$ $=0$ holds,

$$
\begin{aligned}
-\log \frac{\operatorname{det} \nabla_{\omega}^{2}}{\operatorname{det} \nabla_{0}^{2}} & =-\frac{1}{12 \pi} \int_{M} \omega\left\{(J d v)_{\omega}+(J d v)_{0}\right\} \\
& =-\frac{1}{12 \pi} \int_{M} \omega\left(\Delta_{0} \omega\right)(d v)_{0}-\frac{1}{6 \pi} \int_{M} \omega(J d v)_{0} .
\end{aligned}
$$

The scale invariant determinant functional is

$$
-\log \frac{\mathscr{D}\left(\not^{2}, e^{2 \omega} g_{0}\right)}{\mathscr{D}\left(\nabla^{2}, g_{0}\right)}=\log \frac{\mathscr{D}\left(\Delta, e^{2 \omega} g_{0}\right)}{\mathscr{D}\left(\Delta, g_{0}\right)} \text {. }
$$

As a result, on $S^{2}$, det $\nabla_{\omega}^{2}$ is minimized for $g=g_{\omega}=e^{2 \omega} g_{0} \in\left[g_{0}\right]$ of volume $4 \pi$ exactly when $g_{\omega}=h \cdot g_{0}$ for some $h \in \operatorname{ctran}\left(S^{2}, g_{0}\right)$.

Again, the absence of negative eigenvalues allows us to reduce to the extremal problem for the scale invariant determinant functional. Note that though the construction of the spinor bundle depends on the metric, spinor bundles constructed from conformally related metrics can be identified.

For $m \geq 4$, the space $L_{4}^{m}$ of level 4 local scalar $\mathrm{O}(m)$ invariants is fourdimensional. A basis which is especially adapted to dimension independent calculations is $|R|^{2},|\rho|^{2}, \tau^{2}, \Delta \tau$. A convenient basis for calculations involving conformal change of metric is $|C|^{2},|V|^{2}, J^{2}, \Delta J$; this new basis can be 
written in terms of the old one using coefficients that are rational in $m$; thus we have two different bases of $\mathscr{L}_{4}$ over Rat $(m)$. In the conformally flat category, $|V|^{2}, J^{2}$ is a basis of $\tilde{\mathscr{L}}_{4} / \delta \tilde{\mathscr{L}}_{3,1}$ over Rat $(m)$. By, e.g., [Bra1],

$$
\begin{aligned}
\left.(d / d \varepsilon)\right|_{\varepsilon=0}\left(J^{2}\right)_{\varepsilon \omega} & =-4 \omega\left(J^{2}\right)_{0}+2 J \Delta \omega, \\
\left.(d / d \varepsilon)\right|_{\varepsilon=0}\left(|V|^{2}\right)_{\varepsilon \omega} & =-4 \omega\left(|V|^{2}\right)_{0}-2\left\langle V, \operatorname{Hess}_{g_{0}} \omega\right\rangle
\end{aligned}
$$

where for two-tensors $\varphi$ and $\psi,\langle\varphi, \psi\rangle=\varphi_{i j} \psi_{i j}$ in an orthonormal basis. By the Bianchi identity $V_{i j \mid i}=J_{\mid j}$ and integration by parts, the space $C \tilde{L}_{4}^{4} / \delta \tilde{L}_{3,1}^{4}$ in dimension 4 has the single generator $\left[J^{2}-|V|^{2}\right]=J^{2}-|V|^{2}+\delta \tilde{L}_{3,1}^{4}$, of which $[Q]$ and $\left[\mathrm{Pff}_{4}\right]$ must be scalar multiples. Here, as in Sec. 1, we write $Q=Q_{4}$. (That $C \tilde{L}_{4}^{4} / \delta \tilde{L}_{3,1}^{4}$ is one-dimensional also follows from the general result [BGP, Theorem 0.2] described at the end of Remark 5.5.) On $S^{4}$ with its standard metric, $J^{2}-|V|^{2}=3, \mathrm{Pff}_{4}=2 / v_{4}=3 / 4 \pi^{2}$, and by Theorem $2.8(\mathrm{f})$, $Q=6 ;$ thus

$$
2\left[J^{2}-|V|^{2}\right]=[Q]=8 \pi^{2}\left[\mathrm{Pff}_{4}\right] \quad \text { in } C \tilde{L}_{4}^{4} / \delta \tilde{F}_{3}^{4} .
$$

By Remark 5.5, the technical assumption 5.1(b) will automatically be satisfied for the pair $(4, A)$ provided $A$ satisfies $4.1,4.3$, and 4.6, and $(4, A)$ satisfies $5.1(\mathrm{a})$. In this case we can write

$$
u_{A}(m) a_{4}[A]=\int\left\{\alpha_{4}(m) Q+(m-4)\left(k_{V}(m)|V|^{2}+k_{J}(m) J^{2}\right)\right\} d v
$$

for some rational functions $k_{V}(m)$ and $k_{J}(m)$ which are regular at $m=4$. Here, as in Sec. 1, $Q=Q_{4}$. Now take a particular 4-manifold $\left(M, g_{0}\right)$, and assume for simplicity that $\mathscr{N}(A)=0$ there. The conformal index is

$$
\zeta_{A}(0)=a_{4}[A]=u_{A}(4)^{-1} \alpha_{4}(4) \int_{M} Q d v=8 \pi^{2} u_{A}(4)^{-1} \alpha_{4}(4) \chi(M)
$$

specializing Theorem 5.6, we get

Theorem 5.11 [BØ3]. Suppose $A=A(m)$ satisfies 4.1, 4.3, and 4.6, and is regular at $m=4$. Suppose that the pair $(4, A)$ satisfies $5.1(\mathrm{a})$. Let $\left(M, g_{0}\right)$ be a particular conformally flat compact Riemannian four-manifold (with spin structure if required to define $A)$, and suppose that $\mathscr{N}(A)=0$ on $\left(M, g_{0}\right)$. Then

$$
\begin{aligned}
-(2 \ell)^{-1} u_{A}(4) \log \frac{\operatorname{det} A_{\omega}}{\operatorname{det} A_{0}} \\
=\frac{1}{2} \alpha_{4}(4) \int_{M} \omega\left\{(Q d v)_{\omega}+(Q d v)_{0}\right\} \\
\quad+k_{V}(4) \int_{M}\left\{\left(|V|^{2} d v\right)_{\omega}-\left(|V|^{2} d v\right)_{0}\right\}+k_{J}(4) \int_{M}\left\{\left(J^{2} d v\right)_{\omega}-\left(J^{2} d v\right)_{0}\right\} \\
=\alpha_{4}(4)\left\{\frac{1}{2} \int_{M} \omega\left(P_{0} \omega\right)(d v)_{0}+\int_{M} \omega(Q d v)_{0}\right\} \\
\quad+\left(k_{V}(4)+k_{J}(4)\right) \int_{M}\left\{\left(J^{2} d v\right)_{\omega}-\left(J^{2} d v\right)_{0}\right\}
\end{aligned}
$$


The scale invariant determinant functional is

$$
\begin{aligned}
-(2 \ell)^{-1} u_{A}(4) \log \frac{\mathscr{D}\left(A, e^{2 \omega} g_{0}\right)}{\mathscr{D}\left(A, g_{0}\right)}= & -2 \pi^{2} \alpha_{4}(4) \chi(M) \log \frac{\int_{M} e^{4 \omega}(d v)_{0}}{\operatorname{vol}\left(g_{0}\right)} \\
& -(2 \ell)^{-1} u_{A}(4) \log \frac{\operatorname{det} A_{\omega}}{\operatorname{det} A_{0}} .
\end{aligned}
$$

If $Q$ is constant on $\left(M, g_{0}\right), Q_{0}=8 \pi^{2} \chi(M) / \operatorname{vol}\left(g_{0}\right)$, and

$$
\begin{aligned}
-(2 \ell)^{-1} u_{A}(4) \log \frac{\mathscr{D}\left(A, e^{2 \omega} g_{0}\right)}{\mathscr{D}\left(A, g_{0}\right)} \\
=\alpha_{4}(4)\left\{-\frac{Q_{0} \operatorname{vol}\left(g_{0}\right)}{4} \log \frac{\int_{M} e^{4(\omega-\omega)}(d v)_{0}}{\operatorname{vol}\left(g_{0}\right)}+\frac{1}{2} \int_{M} \omega\left(P_{0} \omega\right)(d v)_{0}\right\} \\
\quad+\left(k_{V}(4)+k_{J}(4)\right) \int_{M}\left\{\left(J^{2} d v\right)_{\omega}-\left(J^{2} d v\right)_{0}\right\} .
\end{aligned}
$$

If in addition $J$ is constant on $\left(M, g_{0}\right)$,

$$
\begin{aligned}
& -(2 \ell)^{-1} u_{A}(4) \log \frac{\mathscr{D}\left(A, e^{2 \omega} g_{0}\right)}{\mathscr{D}\left(A, g_{0}\right)} \\
& =\alpha_{4}(4)\left\{-\frac{Q_{0} \operatorname{vol}\left(g_{0}\right)}{4} \log \frac{\int_{M} e^{4(\omega-\omega)}(d v)_{0}}{\operatorname{vol}\left(g_{0}\right)}+\frac{1}{2} \int_{M} \omega\left(P_{0} \omega\right)(d v)_{0}\right\} \\
& +\left(k_{V}(4)+k_{J}(4)\right) \int_{M}\left\{\left(\frac{(\Delta+J)_{0} e^{\omega}}{e^{\omega}}\right)^{2}-J_{0}^{2}\right\}(d v)_{0} .
\end{aligned}
$$

In particular, if $g_{0}$ is the standard $S^{4}$ metric,

$$
\begin{aligned}
-(2 \ell)^{-1} & u_{A}(4) \log \frac{\mathscr{D}\left(A, e^{2 \omega} g_{0}\right)}{\mathscr{D}\left(A, g_{0}\right)} \\
= & 4 \pi^{2} \alpha_{4}(4)\left\{-\log \int_{S^{4}} e^{4(\omega-\omega)} d \xi+\frac{1}{3} \int_{S^{4}} \omega\left(\Delta_{0}\left(\Delta_{0}+2\right) \omega\right) d \xi\right\} \\
& +\frac{8 \pi^{2}\left(k_{V}(4)+k_{J}(4)\right)}{3} \int_{S^{4}}\left\{\left(\frac{\left(\Delta_{0}+2\right) e^{\omega}}{e^{\omega}}\right)^{2}-4\right\} d \xi .
\end{aligned}
$$

Here we have used the conformal index property in the form (5.8) to eliminate the more complicated invariant $|V|^{2}$ from the formulas in favor of $J^{2}$. For (5.9), we have used the covariance relation for the conformal Laplacian $Y$, together with the fact that $Y=\Delta+J$ in dimension four. For (5.10), we have substituted the standard four-sphere values $Q=6, J=2$, and $P=\Delta(\Delta+2)$.

Remark 5.12. In [BØ3], where a somewhat different computational approach was taken, the numbers $u_{A}(4)^{-1} \alpha_{4}(4)$ and $u_{A}(4)^{-1}\left(k_{V}(4)+k_{J}(4)\right)$ were called $\frac{1}{2} \beta_{2}$ and $\frac{1}{2} \beta_{3}$ respectively; this notation is also used in [BCY]. In [BØ3], $\beta_{3}$ arose as the coefficient of $\Delta J$ in $U_{4}[A] . \quad \beta_{3}$ ends up attached to the $J^{2}$ term in the determinant formula because $\frac{1}{2} J^{2}$ is a conformal primitive for $\Delta J$. In the current approach, we do not need to compute such divergence terms, but need instead knowledge of all the non-divergence terms as functions of the dimension $m$, not just in dimension four. In essence, instead of computing divergence terms via the "reconstruction principle" of Corollary 4.9 , analytically 
continuing to dimension 4 , and then finding a conformal primitive, we just find a conformal primitive for $m \neq 4$ and analytically continue to $m=4$.

Just as in dimension 2, we now have everything written in terms of functionals which are sharply estimated by Lieb-Beckner inequalities, at least on the sphere. The novelty is that now we have a linear combination of two such functionals, that associated to the embedding $L_{2}^{2} \hookrightarrow e^{L}$, and that associated to the "ordinary" borderline Sobolev inequality $L_{1}^{2} \hookrightarrow L^{4}$. The best constants and extremals are given by Corollary 3.6 and Theorem 3.7. However, we are not finished estimating unless the coefficients involved, $\alpha_{4}(4)$ and $k_{V}(4)+k_{J}(4)$, have the same sign, or else one vanishes and the other does not. Fortunately, the signs do agree in the cases $A=Y$ and $A=\not^{2}$.

To find these coefficients, recall from [G2, Theorem 4.3] that

$$
\begin{aligned}
(4 \pi)^{m / 2} 360 a_{4}\left[\nabla^{*} \nabla-\mathscr{E}\right] & \\
= & \int_{M} \operatorname{tr}_{\mathbf{v}}\left\{5 \tau^{2}-2|\rho|^{2}+2|R|^{2}+60 \tau \mathscr{E}+180 \mathscr{E}^{2}+30 W_{i j} W_{i j}\right\} d v,
\end{aligned}
$$

where $W$ is the curvature of the bundle $\mathbb{V}$ in which $\nabla^{*} \nabla-\mathscr{E}$ acts. (Recall that our convention on index placement in the Riemann tensor differs from that of the reference; on standard $S^{2}$, our $R_{1212}$ is positive.) An expression like $W_{i j} W_{i j}$ does not, of course, refer to any particular orthonormal frame, but is an abbreviation for that quantity that equals $W_{i j} W_{i j}$ in any local orthonormal frame; that is, it is invariant index notation. We apply (5.11) to the Yamabe operator in the conformally flat category via Table 5.1, which expresses all relevant invariants in terms of $J^{2}$ and $|V|^{2}$.

TABLE 5.1. Terms in $(4 \pi)^{m / 2} 360 a_{4}[Y]\left(\mathscr{E}=-\frac{1}{2}(m-2) J, W=0\right)$

\begin{tabular}{|l|c|c|c|c|}
\hline & $J^{2}$ & $|V|^{2}$ & & coef. \\
\hline$\tau^{2}$ & $4(m-1)^{2}$ & 0 & & 5 \\
\hline$|\rho|^{2}$ & $3 m-4$ & $(m-2)^{2}$ & & -2 \\
\hline$|R|^{2}$ & 4 & $4(m-2)$ & & 2 \\
\hline$\tau \mathscr{E}$ & $-(m-1)(m-2)$ & 0 & & 60 \\
\hline $\mathscr{E}^{2}$ & $\frac{1}{4}(m-2)^{2}$ & 0 & & 180 \\
\hline
\end{tabular}

The meaning of, for example, the second row of the table, is that

$$
|\rho|^{2}=(3 m-4) J^{2}+(m-2)^{2}|V|^{2},
$$

and that the coefficient of $|\rho|^{2}$ on the right in (5.11) is -2 . Thus, since

$$
\int Q d v=\int\left\{\frac{1}{2} m J^{2}-2|V|^{2}\right\} d v
$$

we have

$$
\begin{aligned}
(4 \pi)^{m / 2} 360 a_{4}[Y] & =(m-6) \int\left\{(5 m-16) J^{2}-2(m-2)|V|^{2}\right\} d v \\
& =2(m-6) \int Q d v+(m-4)(m-6) \int\left\{4 J^{2}-2|V|^{2}\right\} d v .
\end{aligned}
$$

We collect this information in: 
Lemma 5.13. For $(n, A)=(4, Y)$ and the choice $u_{Y}(m)=(4 \pi)^{m / 2}$, the function in 5.1(b) is

$$
\alpha_{4}(m)=(m-6) / 180,
$$

and the local invariant can be taken to be

$$
B_{4}(m)=(m-6)\left\{4 J^{2}-2|V|^{2}\right\} / 360 .
$$

As a result, $\alpha_{4}(4)=k_{V}(4)+k_{J}(4)=-1 / 90$.

Remark 5.14. The factor $m-6$ in the formula for $a_{4}[Y]$ is expected. By a result that appears to have originated in [Sc] (see also [BØ1, BG1]), $U_{m-2}[Y]$ is a local conformal invariant in the sense that

$$
\bar{g}=\Omega^{2} g, 0<\Omega \in C^{\infty}(M) \Rightarrow \bar{U}_{m-2}[Y]=\Omega^{2-m} U_{m-2}[Y] .
$$

(A similar result holds for any conformal covariant $A$ of order $2 \ell$ satisfying 4.1 and 4.3, replacing each $m-2$ above with $m-2 \ell$. This is most clearly seen from [BG1, Lemma 3.1(c)].) But we are now working in the conformally flat category, where all such local conformal invariants vanish. The same principle can be seen in the factor of $m-4$ in (5.4) above, and in the factor of $m-8$ in Lemma 6.9 below.

To treat $A=\nabla^{2}$, we need to adjust the above calculation for the change from $\mathscr{E}=-(m-2) J / 2$ to $\mathscr{E}=-\tau / 4=-(m-1) J / 2$ (the Lichnerowicz formula), for the change in fiber dimension, and for the spin curvature; these adjustments are detailed in Table 5.2.

TABLE 5.2 (value at $\left.\mathscr{E}=-\frac{1}{2}(m-1) J\right)$ minus (value at $\mathscr{E}=-\frac{1}{2}(m-2) J$ )

\begin{tabular}{|c|c|c|c|c|}
\hline & $J^{2}$ & $|V|^{2}$ & & coef. \\
\hline$\tau \mathscr{E}$ & $-(m-1)$ & 0 & & 60 \\
\hline $\mathscr{E}^{2}$ & $\frac{1}{4}(2 m-3)$ & 0 & & 180 \\
\hline
\end{tabular}

New term in $(4 \pi)^{m / 2} 360 \cdot 2^{-[m / 2]} a_{4}\left[\not{ }^{2}\right]$ resulting from spinor $(\Sigma)$ bundle curvature $W_{i j}=-\frac{1}{4} R_{k l i j} \gamma_{k} \gamma_{l}$

\begin{tabular}{|c|c|c|c|c|}
\hline & $J^{2}$ & $|V|^{2}$ & & coef. \\
\hline $\operatorname{tr}_{\Sigma} W_{i j} W_{i j}$ & $-\frac{1}{2}$ & $-\frac{1}{2}(m-2)$ & & 30 \\
\hline
\end{tabular}

Collecting the information, we get:

Lemma 5.15. For $(n, A)=\left(4, \nabla^{2}\right)$, with the choice $u_{\nabla^{2}}(m)=(4 \pi)^{m / 2} 2^{-m / 2}$ ( $m$ even), the function in (5.2) can be taken to be

$$
\alpha_{4}(m)=11 / 360
$$

and the local invariant can be taken to be

$$
B_{4}(m)=\frac{\frac{1}{2}(10 m-3) J^{2}-(2 m+7)|V|^{2}}{360} .
$$

As a result, $\alpha_{4}(4)=11 / 360$ and $k_{V}(4)+k_{J}(4)=7 / 720$. In particular, these numbers have the same sign.

Neither $Y$ nor $\not^{2}$ has negative eigenvalues on $\left(S^{4}, g_{0}\right)$; thus by Theorem 4.8 , the same is true with $g_{\omega}$ in place of $g_{0}$ for any $\omega \in C^{\infty}\left(S^{4}\right)$. Thus, 
the extremal problem for $\operatorname{det} Y_{\omega}$ is the same as that for $\log \left(\mathscr{D}\left(Y, e^{2 \omega} g_{0}\right)\right) /$ $\left(\mathscr{D}\left(Y, g_{0}\right)\right)$, and similarly for det $\not^{2}$. (If there were an odd number of negative eigenvalues, the extremal problem would be the same, but the roles of maxima and minima would be reversed.) In view of Theorem 5.11, Corollary 3.6, and Theorem 3.7, we have proved:

Theorem 5.16. On $S^{4}$, $\operatorname{det} Y_{\omega}$ is minimized for $g=g_{\omega}=e^{2 \omega} g_{0} \in\left[g_{0}\right]$ of volume $8 \pi^{2} / 3$ exactly when $g_{\omega}=h \cdot g_{0}$ for some $h \in \operatorname{ctran}\left(S^{4}, g_{0}\right) . \operatorname{det} \nabla_{\omega}^{2}$ is maximized at exactly the same metrics.

\section{THE FUNCTIONAL DETERMINANT IN DIMENSION 6}

One of the requirements for an application of our method to dimension 6 is a formula for $\int Q_{6} d v$ in the conformally flat case. Actual computation of the operator $P_{6}$ via the algorithm implicit in [GJMS], or in [Wü], is a formidable undertaking. Instead of taking this tack, we shall write down a trial formula, with undetermined coefficients, for $Q_{6}$, then evaluate the coefficients using some special computations.

Lemma 6.1. The four invariants $|d J|^{2}, J^{3}, J|V|^{2}$, and $\operatorname{tr} V^{3}:=V_{i j} V_{j k} V_{k i}$ give a basis of $\tilde{\mathscr{L}}_{6} / \delta \mathscr{L}_{5,1}$.

Proof. A basis of $\mathscr{L}_{6}$ is given in [G2]. To get the above result, one restricts to the conformally flat case, and shows that each of the restricted invariants is a linear combination of the four given above, and an exact divergence. That this program can be carried out follows from the formula (1.8) for $R$ in terms of $C$ and $V$, plus Remark 5.4. (Exact formulas are given in Tables 6.1 and 6.2 below.)

Note that the "trace" in $\operatorname{tr} V^{3}$ is that of End $T M$ (or End $T^{*} M$ ). We already know that the $|d J|^{2}$ coefficient in $Q_{6}$ is $(m-6) / 2$ by Corollary 1.6 . We can get more information by evaluating on the standard sphere, and (recalling Remark 1.2) on the conformally flat pseudo-Riemannian manifolds $\left(S^{(q, p)}, g_{0}\right):=\left(S^{q} \times\right.$ $\left.S^{p}, g_{S^{q}}-g_{S^{p}}\right)$, with $p+q=n$. Up to covering, these are the conformal compactifications of the standard signature $(q, p)$ flat spaces; that is, they bear the same relation to these flat spaces as the sphere does to Euclidean space. The group $\mathrm{SO}_{0}(q+1, p+1)$ acts on $\left(S^{(q, p)}, g_{0}\right)$ by conformal transformations [Kl, Bra2], and one can study the representation theoretic problem analogous to that of Section 2 above. We shall not need a full treatment of this theory, but just the analogue of Theorem 2.8(f). Using (2.10) on $S^{q}$ and $S^{p}$ to interpret and rewrite results of Molčanov [M], we get

Theorem $6.2[\mathrm{M}]$. Let $B$ be the operator of (2.14) on standard $S^{q}$ (with $q$ in place of $m$ ), and let $C$ be the similar operator on standard $S^{p}$ (with $p$ in place of $m$ ). Then the unique differential intertwinor of order $n \in 2 \mathbb{Z}^{+}$for the conformal action of $\mathrm{SO}_{0}(q+1, p+1)$ on functions over $S^{(q, p)}$ is

$$
P_{n}=P_{2} \prod_{\alpha=1}^{\frac{n-2}{4}}\{(B+C+2 \alpha)(B-C-2 \alpha)(B+C-2 \alpha)(B-C+2 \alpha)\}
$$


if $n / 2$ is odd, where $P_{2}=(B+C)(B-C)$, and

$P_{n}=\prod_{\alpha=1}^{\frac{n}{4}}\{(B+C+(2 \alpha-1))(B-C-(2 \alpha-1))(B+C-(2 \alpha-1))(B-C+(2 \alpha-1))\}$

if $n / 2$ is even.

Note that if $p=0$, we are (up to covering) in the situation of Theorem 2.8(f), and we recover that result by noting that the operator $C$ is just the number $1 / 2$.

Corollary 6.3. In the setting of Theorem 6.2,

$$
32 Q_{6}=(m+2)(m-2)(q-p)(q-p+4)(q-p-4) .
$$

Proof. We compute using $(m-6) Q_{6} / 2=P_{6} 1$.

On $\left(S^{(q, p)}, g_{0}\right)$,

$$
d J=0, \quad 8 J^{3}=(q-p)^{3}, \quad 8 J|V|^{2}=m(q-p), \quad 8 \operatorname{tr} V^{3}=q-p .
$$

As a result, if

$$
\int Q_{6} d v=\int\left\{\frac{m-6}{2}|d J|^{2}+a(m) J^{3}+b(m) J|V|^{2}+c(m) \operatorname{tr} V^{3}\right\} d v
$$

(using the $|d J|^{2}$ coefficient determined above), then equating coefficients of $(q-p)^{3}$ and of $q-p$ gives

$$
\begin{aligned}
4 a(m) & =(m+2)(m-2), \\
m b(m)+c(m) & =-4(m+2)(m-2) .
\end{aligned}
$$

Thus our consideration of mixed signature conformally flat spaces has given us two of the three remaining ( $m$ dependent) undetermined coefficients; the sphere alone provides just one. To find the remaining undetermined coefficient, we do a partial computation of the integrals of $Q_{6},|d J|^{2}, J^{3}, J|V|^{2}$, and $\operatorname{tr} V^{3}$ on the Riemannian manifolds $\left(T^{m}, e^{2 \omega} g_{0}\right)$, where $\left(T^{m}, g_{0}\right)$ is the flat torus and $\omega \in C^{\infty}\left(T^{m}\right)$. Direct computation gives

$$
\begin{aligned}
J_{\omega} & =-e^{-2 \omega} \operatorname{tr} \lambda \\
V_{\omega} & =-\lambda \\
d J_{\omega} & =e^{-2 \omega}\left\{d \Delta \omega-2(\Delta \omega) d \omega+\frac{m-2}{2} d|d \omega|^{2}+(m-2)|d \omega|^{2} d \omega\right\}
\end{aligned}
$$

where all natural differential operators are computed in the background metric $g_{0}$, and

$$
\lambda=\operatorname{Hess} \omega-d \omega \otimes d \omega+\frac{1}{2}|d \omega|^{2} g_{0} .
$$

Now introduce "..." as an abbreviation for "differential monomials of fourth or higher degree in $\omega . "$ We have

$$
\begin{aligned}
J_{\omega}^{3} & =e^{-6 \omega}\left((\Delta \omega)^{3}+\ldots\right), \\
\left(J|V|^{2}\right)_{\omega} & =e^{-6 \omega}\left((\Delta \omega)|\operatorname{Hess} \omega|^{2}+\ldots\right), \\
\left(\operatorname{tr} V^{3}\right)_{\omega} & =-e^{-6 \omega}\left(\operatorname{tr}(\operatorname{Hess} \omega)^{3}+\ldots\right), \\
\left(|d J|^{2}\right)_{\omega} & =e^{-6 \omega}\left(|d \Delta \omega|^{2}+2\left\langle d \Delta \omega,-2(\Delta \omega) d \omega+\frac{m-2}{2} d|d \omega|^{2}\right\rangle+\ldots\right) .
\end{aligned}
$$


Since $(d v)_{\omega}=e^{m \omega}(d v)_{0}$, the integrated form of this is

$$
\int\left(J^{3} d v\right)_{\omega}=\int e^{2 \beta \omega}\left((\Delta \omega)^{3}+\ldots\right)(d v)_{0}
$$

where $\beta=(m-6) / 2$, and similarly for the other invariants. Integration by parts gives several relations among the integrals of invariants appearing in (6.4):

$$
\begin{aligned}
& \int e^{2 \beta \omega}(\Delta \omega)\langle d \Delta \omega, d \omega\rangle(d v)_{0}=\int e^{2 \beta \omega}\left\{\frac{1}{2}(\Delta \omega)^{3}+\ldots\right\}(d v)_{0}, \\
& \int e^{2 \beta \omega}\left\langle d \Delta \omega, d|d \omega|^{2}\right\rangle(d v)_{0}=\int e^{2 \beta \omega}\left\{2(\Delta \omega)|\operatorname{Hess} \omega|^{2}-(\Delta \omega)^{3}+\ldots\right\}(d v)_{0}, \\
& \int e^{2 \beta \omega}\left(\operatorname{tr}(\operatorname{Hess} \omega)^{3}\right)(d v)_{0}=\int e^{2 \beta \omega}\left\{\frac{1}{2}(\Delta \omega)^{3}-\frac{3}{2}(\Delta \omega)|\operatorname{Hess} \omega|^{2}+\ldots\right\}(d v)_{0} .
\end{aligned}
$$

As a result,

$$
\begin{aligned}
\int e^{2 \beta \omega}\left(J^{3} d v\right)_{\omega} & =\int e^{2 \beta \omega}\left\{(\Delta \omega)^{3}+\ldots\right\}(d v)_{0} \\
\int e^{2 \beta \omega}\left(J|V|^{2} d v\right)_{\omega} & =\int e^{2 \beta \omega}\left\{(\Delta \omega)|\operatorname{Hess} \omega|^{2}+\ldots\right\}(d v)_{0} \\
\int e^{2 \beta \omega}\left(\operatorname{tr} V^{3} d v\right)_{\omega} & =\int e^{2 \beta \omega}\left\{-\frac{1}{2}(\Delta \omega)^{3}+\frac{3}{2}(\Delta \omega)|\operatorname{Hess} \omega|^{2}+\ldots\right\}(d v)_{0}, \\
\int e^{2 \beta \omega}\left(|d J|^{2} d v\right)_{\omega} & =\int e^{2 \beta \omega}\left\{|d \Delta \omega|^{2}-m(\Delta \omega)^{3}\right. \\
& \left.+2(m-2)(\Delta \omega)|\operatorname{Hess} \omega|^{2}+\ldots\right\}(d v)_{0} .
\end{aligned}
$$

By (1.11), the fact that $P_{6}=\Delta^{3}$ on $\left(T^{m}, g_{0}\right)$, and integration by parts,

$$
\beta \int\left(Q_{6} d v\right)_{\omega}=\int e^{\beta \omega}\left(\Delta^{3} e^{\beta \omega}\right)(d v)_{0}=\int\left|d \Delta e^{\beta \omega}\right|^{2}(d v)_{0}
$$

But

$$
d \Delta e^{\beta \omega}=e^{\beta \omega}\left(\beta d \Delta \omega-\beta^{2} d|d \omega|^{2}+\beta^{2}(\Delta \omega) d \omega-\beta^{3}|d \omega|^{2} d \omega\right) .
$$

Integrating by parts and using (6.5), we get

$\beta \int\left(Q_{6} d v\right)_{\omega}=\int e^{2 \beta \omega}\left\{\beta^{2}|d \Delta \omega|^{2}-\beta^{3}(\Delta \omega)^{3}+4 \beta^{3}(\Delta \omega)|\operatorname{Hess} \omega|^{2}+\ldots\right\}(d v)_{0}$.

Moreover, it is easily seen that the invariant integrals $\int e^{2 \beta \omega} S_{k}(\omega)(d v)_{0}$, where $S_{1}(\omega)=|d \Delta \omega|^{2}, S_{2}(\omega)=(\Delta \omega)^{3}$, and $S_{3}(\omega)=(\Delta \omega) \mid$ Hess $\left.\omega\right|^{2}$, are linearly independent. In the notation of $(6.1)$, the result is that

$$
b(m)=-4 m, \quad c(m)=16 .
$$

Here we have used (6.2), but not our knowledge of the $|d J|^{2}$ coefficient $\beta$, nor (6.3); these can be considered as checks on our calculations. We pause to collect the information: 
Theorem 6.4. In the conformally flat category,

$$
\int Q_{6} d v=\int\left\{\frac{m-6}{2}|d J|^{2}+\frac{(m+2)(m-2)}{4} J^{3}-4 m J|V|^{2}+16 \operatorname{tr} V^{3}\right\} d v .
$$

Another check on this result is the case $m=6$, where it implies that $Q_{6} \simeq 8\left(J^{3}-3 J|V|^{2}+2 \operatorname{tr} V^{3}\right)$ modulo exact divergences for conformally flat metrics. The following lemma shows that up to a constant factor, this is the only possibility.

Lemma 6.5. The space $C \tilde{L}_{6}^{6} / \delta \tilde{L}_{5,1}^{6}$ is generated by the coset $\left[J^{3}-3 J|V|^{2}+\right.$ $\left.2 \operatorname{tr} V^{3}\right]$.

Proof. By [BGP, Theorem 0.2], the space in question is one-dimensional. On standard $S^{6}$, the average (normalized integral) of the invariant $J^{3}-3 J|V|^{2}+$ $2 \operatorname{tr} V^{3}$ in question is $625 / 9$; thus the equivalence class of this invariant is nonzero.

In particular, the Pfaffian must have the form $c\left(J^{3}-3 J|V|^{2}+2 \operatorname{tr} V^{3}\right)$ on conformally flat 6-manifolds, where $c$ is a universal constant. Since $\chi\left(S^{6}\right)=2$ and $J=3, V=\frac{1}{2} g$ on $S^{6}$, we have $15 c v_{6}=2$. Since $v_{6}=16 \pi^{3} / 15$,

$$
\begin{aligned}
\mathrm{Pff}_{6} & =\left(J^{3}-3 J|V|^{2}+2 \operatorname{tr} V^{3}\right) / 8 \pi^{3}, \\
\int Q_{6} d v & =64 \pi^{3} \chi(M)
\end{aligned}
$$

in the conformally flat case.

By Remark 5.5, the technical assumption 5.1(b) will be satisfied for the pair $(6, A)$ provided $A$ satisfies $4.1,4.3$, and 4.6 , and $(6, A)$ satisfies 5.1(a). In this case we can write

$$
\begin{array}{r}
u_{A}(m) a_{6}[A]=\int\left\{\alpha_{6}(m) Q_{6}+(m-6)\left(k_{0}(m)|d J|^{2}+k_{1}(m) J^{3}\right.\right. \\
\left.\left.+k_{2}(m) J|V|^{2}+k_{4}(m) \operatorname{tr} V^{3}\right)\right\} d v
\end{array}
$$

for rational functions $k_{i}(m), i=0, \ldots, 3$, which are regular at $m=6$. Taking a particular conformally flat 6-manifold $\left(M, g_{0}\right)$ on which $\mathscr{N}(A)=0$, the conformal index is

$$
\zeta_{A}(0)=a_{6}[A]=u_{A}(6)^{-1} \alpha_{6}(6) \int_{M} Q_{6} d v=64 \pi^{3} u_{A}(6)^{-1} \alpha_{6}(6) \chi(M) .
$$

Specializing Theorem 5.6, we get:

Theorem 6.6. Suppose $A=A(m)$ satisfies 4.1, 4.3, and 4.6, and is regular at $m=6$. Suppose the pair $(6, A)$ satisfies $5.1(\mathrm{a})$. Let $\left(M, g_{0}\right)$ be a particular conformally flat compact Riemannian six-manifold (with spin structure if 

required to define $A)$, and suppose that $\mathscr{N}(A)=0$ on $\left(M, g_{0}\right)$. Then

$$
\begin{aligned}
& -(2 \ell)^{-1} u_{A}(6) \log \frac{\operatorname{det} A_{\omega}}{\operatorname{det} A_{0}}=\frac{1}{2} \alpha_{6}(6) \int_{M} \omega\left\{\left(Q_{6} d v\right)_{\omega}+\left(Q_{6} d v\right)_{0}\right\} \\
& +k_{0}(6) \int_{M}\left\{\left(|d J|^{2} d v\right)_{\omega}-\left(|d J|^{2} d v\right)_{0}\right\}+k_{1}(6) \int_{M}\left\{\left(J^{3} d v\right)_{\omega}-\left(J^{3} d v\right)_{0}\right\} \\
& +k_{2}(6) \int_{M}\left\{\left(J|V|^{2} d v\right)_{\omega}-\left(J|V|^{2} d v\right)_{0}\right\} \\
& +k_{3}(6) \int_{M}\left\{\left(\operatorname{tr} V^{3} d v\right)_{\omega}-\left(\operatorname{tr} V^{3} d v\right)_{0}\right\} \\
& =\alpha_{6}(6)\left\{\frac{1}{2} \int_{M} \omega\left(\left(P_{6}\right)_{0} \omega\right)(d v)_{0}+\int_{M} \omega\left(Q_{6} d v\right)_{0}\right\} \\
& +k_{0}(6) \int_{M}\left\{\left(|d J|^{2} d v\right)_{\omega}-\left(|d J|^{2} d v\right)_{0}\right\} \\
& +\left(k_{1}(6)-\frac{1}{2} k_{3}(6)\right) \int_{M}\left\{\left(J^{3} d v\right)_{\omega}-\left(J^{3} d v\right)_{0}\right\} \\
& +\left(k_{2}(6)+\frac{3}{2} k_{3}(6)\right) \int_{M}\left\{\left(J|V|^{2} d v\right)_{\omega}-\left(J|V|^{2} d v\right)_{0}\right\}
\end{aligned}
$$

The scale invariant determinant functional is

$$
\begin{aligned}
& -(2 \ell)^{-1} u_{A}(6) \log \frac{\mathscr{D}\left(A, e^{2 \omega} g_{0}\right)}{\mathscr{D}\left(A, g_{0}\right)} \\
& \quad=-\frac{32 \pi^{3}}{3} \alpha_{6}(6) \chi(M) \log \frac{\int_{M} e^{6 \omega}(d v)_{0}}{\operatorname{vol}\left(g_{0}\right)}-(2 \ell)^{-1} u_{A}(6) \log \frac{\operatorname{det} A_{\omega}}{\operatorname{det} A_{0}} .
\end{aligned}
$$

If $Q_{6}$ is constant on $\left(M, g_{0}\right)$,

$$
\begin{aligned}
& -(2 \ell)^{-1} u_{A}(6) \log \frac{\mathscr{D}\left(A, e^{2 \omega} g_{0}\right)}{\mathscr{D}\left(A, g_{0}\right)} \\
& =\alpha_{6}(6)\left\{-\frac{\left(Q_{6}\right)_{0} \operatorname{vol}\left(g_{0}\right)}{6} \log \frac{\int_{M} e^{6(\omega-\omega)}(d v)_{0}}{\operatorname{vol}\left(g_{0}\right)}+\frac{1}{2} \int_{M} \omega\left(\left(P_{6}\right)_{0} \omega\right)(d v)_{0}\right\} \\
& \quad+k_{0}(6) \int_{M}\left\{\left(|d J|^{2} d v\right)_{\omega}-\left(|d J|^{2} d v\right)_{0}\right\} \\
& \quad+\left(k_{1}(6)-\frac{1}{2} k_{3}(6)\right) \int_{M}\left\{\left(J^{3} d v\right)_{\omega}-\left(J^{3} d v\right)_{0}\right\} \\
& \quad+\left(k_{2}(6)+\frac{3}{2} k_{3}(6)\right) \int_{M}\left\{\left(J|V|^{2} d v\right)_{\omega}-\left(J|V|^{2} d v\right)_{0}\right\}
\end{aligned}
$$


In particular, if $g_{0}$ is the standard metric on $S^{6}$,

$$
\begin{aligned}
-(2 \ell)^{-1} u_{A}(6) \log \frac{\mathscr{D}\left(A, e^{2 \omega} g_{0}\right)}{\mathscr{D}\left(A, g_{0}\right)} \\
=\frac{64 \pi^{3}}{3} \alpha_{6}(6)\left\{-\log \int_{S^{6}} e^{6(\omega-\omega)} d \xi+\frac{1}{40} \int_{S^{6}} \omega\left(\Delta_{0}\left(\Delta_{0}+4\right)\left(\Delta_{0}+6\right) \omega\right) d \xi\right\} \\
\quad+\frac{16 \pi^{3}}{15}\left\{k_{0}(6) \int_{S^{6}}\left\{\left(|d J|^{2} d \xi\right)_{\omega}-\left(|d J|^{2} d \xi\right)_{0}\right\}\right. \\
\quad+\left(k_{1}(6)-\frac{1}{2} k_{3}(6)\right) \int_{S^{6}}\left\{\left(J^{3} d \xi\right)_{\omega}-\left(J^{3} d \xi\right)_{0}\right\} \\
\left.\quad+\left(k_{2}(6)+\frac{3}{2} k_{3}(6)\right) \int_{S^{6}}\left\{\left(J|V|^{2} d \xi\right)_{\omega}-\left(J|V|^{2} d \xi\right)_{0}\right\}\right\}
\end{aligned}
$$

The remaining step in making the determinant functionals for $Y$ and $\not^{2}$ explicit in dimension 6 is the determination of the constants $\alpha_{6}(6)$ and $k_{1}(6)-$ $\frac{1}{2} k_{3}(6), k_{2}(6)+\frac{3}{2} k_{3}(6)$ in each case. As in the two- and four-dimensional cases, these constants are implicit in an integrated heat invariant, in this case $a_{6}$. By [G2], in the notation of (5.11),

$$
\begin{aligned}
(4 \pi)^{m / 2} 7 ! a_{6}\left[\nabla^{*} \nabla-\mathscr{E}\right]=\int \operatorname{tr}\left\{-11|\nabla \tau|^{2}+6|\nabla \rho|^{2}-28 \rho_{j k \mid l} \rho_{j l \mid k}\right. \\
\quad-3|\nabla R|^{2}-420|\nabla \mathscr{E}|^{2}-168\langle\nabla \tau, \nabla \mathscr{E}\rangle-84 W_{i j \mid j} W_{i k \mid k} \\
\quad+\frac{35}{9} \tau^{3}-\frac{14}{3} \tau|\rho|^{2}+\frac{14}{3} \tau|R|^{2}-\frac{208}{9} \operatorname{tr} \rho^{3}+\frac{64}{3} \rho_{i j} \rho_{k l} R_{i k j l} \\
\quad-\frac{16}{3} \rho_{i j} R_{i k l p} R_{j k l p}+\frac{44}{9} R_{i j k l} R_{i j p q} R_{k l p q}+\frac{80}{9} R_{i j k l} R_{i p k q} R_{j p l q} \\
+840 \mathscr{E}^{3}+420 \tau \mathscr{E}^{2}+70 \tau^{2} \mathscr{E}-28|\rho|^{2} \mathscr{E}+28|R|^{2} \mathscr{E} \\
+56 W_{i j} W_{j k} W_{k i}+70 \tau W_{i j} W_{i j}+56 \rho_{i j} W_{k i} W_{k j} \\
\left.+28 R_{i j k l} W_{i j} W_{k l}+420 \mathscr{E} W_{i j} W_{i j}\right\} d v .
\end{aligned}
$$

Conformal flatness is not assumed in (6.6).

Remark 6.7. For arbitrary $(\mathbb{V}, \nabla)$, the Bianchi identity and integration by parts give:

$$
\begin{aligned}
\int \operatorname{tr}_{\mathbf{V}} W_{i j \mid k} W_{i j \mid k} d v=\int\{ & 2 \operatorname{tr}_{\mathbf{V}} W_{i j \mid j} W_{i k \mid k}-2 \rho_{i j} \operatorname{tr}_{\mathbf{V}} W_{k i} W_{k j} \\
& \left.+R_{i j k l} \operatorname{tr}_{\mathbf{V}} W_{i j} W_{k l}-4 \operatorname{tr}_{\mathbf{V}} W_{i j} W_{j k} W_{k i}\right\} d v .
\end{aligned}
$$

Conformal flatness is not assumed here either. Because of this identity, the expression $W_{i j \mid k} W_{i j \mid k}$ does not occur in (6.6).

Moving to the conformally flat case, we first write the purely metric terms (i.e., those not involving $\mathscr{E}$ or $W$ ) in terms of $J$ and $V$. The results are in Tables 6.1 and 6.2 below; the intermediate calculations are contained in the following lemma: 
Lemma 6.8. Suppose $g$ is conformally flat. Then

$$
\begin{aligned}
|\rho|^{2} & =(m-2)^{2}|V|^{2}+(3 m-4) J^{2}, \\
|R|^{2} & =4(m-2)|V|^{2}+4 J^{2}, \\
V_{i j} \rho_{j k} & =(m-2) V_{i j} V_{j k}+J V_{i k}, \\
V_{i j} \rho_{j k} \rho_{k i} & =(m-2)^{2} \operatorname{tr} V^{3}+2(m-2) J|V|^{2}+J^{3}, \\
V_{i j} R_{i k l p} & =-V_{j p} V_{k l}+V_{j l} V_{k p}-V_{i j} V_{i p} g_{k l}+V_{i j} V_{i l} g_{k p}, \\
V_{i j} R_{i k l p} R_{j k l p} & =2(m-4) \operatorname{tr} V^{3}+6 J|V|^{2}, \\
R_{k l j p} R_{i l k p} & =\frac{1}{2} R_{i k l p} R_{j k l p}, \\
V_{i j} V_{k l} R_{j k i l} & =-2 \operatorname{tr} V^{3}+2 J|V|^{2}, \\
V_{i j} \rho_{k l} R_{j k i l} & =-2(m-2) \operatorname{tr} V^{3}+3(m-2) J|V|^{2}+J^{3} .
\end{aligned}
$$

TABLE 6.1. Quadratic metric terms

\begin{tabular}{|l|c|c|c|c|c|c|}
\hline & $\int|d J|^{2}$ & $\int J^{3}$ & $\int J|V|^{2}$ & $\int \operatorname{tr} V^{3}$ & & coef. \\
\hline $\int|\nabla \tau|^{2}$ & $4(m-1)^{2}$ & 0 & 0 & 0 & & -11 \\
\hline $\int|\nabla \rho|^{2}$ & $m(m-1)$ & 0 & $(m-2)^{2}$ & $-m(m-2)^{2}$ & & 6 \\
\hline $\int \rho_{j k \mid l} \rho_{j l \mid k}$ & $(m-1)^{2}$ & 0 & $(m-2)^{2}$ & $-m(m-2)^{2}$ & & -28 \\
\hline $\int|\nabla R|^{2}$ & $4(m-1)$ & 0 & $4(m-2)$ & $-4 m(m-2)$ & & -3 \\
\hline
\end{tabular}

TABLE 6.2. Cubic metric terms

\begin{tabular}{|l|c|c|c|c|}
\hline & $J^{3}$ & $J|V|^{2}$ & $\operatorname{tr} V^{3}$ & coef. \\
\hline$\tau^{3}$ & $8(m-1)^{3}$ & 0 & 0 & $35 / 9$ \\
\hline$\tau|\rho|^{2}$ & $2(m-1)(3 m-4)$ & $2(m-1)(m-2)^{2}$ & 0 & $-14 / 3$ \\
\hline$\tau|R|^{2}$ & $8(m-1)$ & $8(m-1)(m-2)$ & 0 & $14 / 3$ \\
\hline $\operatorname{tr} \rho^{3}$ & $2(2 m-3)$ & $3(m-2)^{2}$ & $(m-2)^{3}$ & $-208 / 9$ \\
\hline$\rho_{i j} \rho_{k l} R_{i k j l}$ & $2(2 m-3)$ & $4(m-2)^{2}$ & $-2(m-2)^{2}$ & $64 / 3$ \\
\hline$\rho_{i j} R_{i k l p} R_{j k l p}$ & 4 & $10(m-2)$ & $2(m-2)(m-4)$ & $-16 / 3$ \\
\hline$R_{i j k l} R_{i j p q} R_{k l p q}$ & 0 & 24 & $8(m-4)$ & $44 / 9$ \\
\hline$R_{i j k l} R_{i p k q} R_{j p l q}$ & 2 & $6(m-3)$ & $-2(3 m-8)$ & $80 / 9$ \\
\hline
\end{tabular}

Concentrating for the moment on the conformal Laplacian $Y$, we compute the $\mathscr{E}$ terms: 
TABLE 6.3. $A=Y: \mathscr{E}=-\frac{m-2}{2} J, W=0$

\begin{tabular}{|l|c|c|c|c|c|}
\hline & $J^{3}$ & $J|V|^{2}$ & $\operatorname{tr} V^{3}$ & & coef. \\
\hline $\mathscr{E}^{3}$ & $-\frac{1}{8}(m-2)^{3}$ & 0 & 0 & & 840 \\
\hline$\tau \mathscr{E}^{2}$ & $\frac{1}{2}(m-1)(m-2)^{2}$ & 0 & 0 & & 420 \\
\hline$\tau^{2} \mathscr{E}$ & $-2(m-1)^{2}(m-2)$ & 0 & 0 & & 70 \\
\hline$|\rho|^{2} \mathscr{E}$ & $-\frac{1}{2}(m-2)(3 m-4)$ & $-\frac{1}{2}(m-2)^{3}$ & 0 & & -28 \\
\hline$|R|^{2} \mathscr{E}$ & $-2(m-2)$ & $-2(m-2)^{2}$ & 0 & & 28 \\
\hline
\end{tabular}

\begin{tabular}{|l|c|c|c|c|c|c|}
\hline & $\int|d J|^{2}$ & $\int J^{3}$ & $\int J|V|^{2}$ & $\int \operatorname{tr} V^{3}$ & & coef. \\
\hline $\int|\nabla \mathscr{E}|^{2}$ & $\frac{1}{4}(m-2)^{2}$ & 0 & 0 & 0 & & -420 \\
\hline $\int\langle\nabla \tau, \nabla \mathscr{E}\rangle$ & $-(m-1)(m-2)$ & 0 & 0 & 0 & & -168 \\
\hline
\end{tabular}

We now add everything up to get:

\section{Lemma 6.9.}

$$
\begin{aligned}
(4 \pi)^{m / 2} 7 ! a_{6}[Y] & \\
= & (m-8) \int\left\{-3(m-6)|d J|^{2}-\frac{1}{9}\left(35 m^{2}-266 m+456\right) J^{3}\right. \\
& \left.\quad+\frac{2}{3}(m-1)(7 m-30) J|V|^{2}-\frac{2}{9}\left(5 m^{2}-2 m-48\right) \operatorname{tr} V^{3}\right\} d v \\
= & -\frac{5}{3}(m-8) \int Q_{6} d v+(m-8)(m-6) \\
& \cdot \int\left\{-\frac{13}{6}|d J|^{2}-\frac{1}{36}(125 m-314) J^{3}\right. \\
& \left.+\frac{2}{3}(7 m-5) J|V|^{2}-\frac{2}{9}(5 m+28) \operatorname{tr} V^{3}\right\} d v .
\end{aligned}
$$

As a result, for $(n, A)=(6, Y)$ and the choice $u_{Y}(m)=(4 \pi)^{m / 2}$, the function in 5.1(b) can be taken to be

$$
\alpha_{6}(m)=-\frac{5(m-8)}{3 \cdot 7 !},
$$

and the local invariant can be taken to be

$$
\begin{aligned}
B_{6}(m)=\frac{m-8}{7 !}\left\{-\frac{13}{6}|d J|^{2}-\frac{1}{36}(125 m-314) J^{3}\right. & +\frac{2}{3}(7 m-5) J|V|^{2} \\
& \left.-\frac{2}{9}(5 m+28) \operatorname{tr} V^{3}\right\} .
\end{aligned}
$$

In particular,

$3 \cdot 7 !\left(\alpha_{6}(6), k_{0}(6), k_{1}(6)-\frac{1}{2} k_{3}(6), k_{2}(6)+\frac{3}{2} k_{3}(6)\right)=(10,13,34,-32)$.

To handle $A=\not^{2}$, we need to adjust the calculation for the change from $\mathscr{E}=-(m-2) J / 2$ to $\mathscr{E}=-(m-1) J / 2$, and take account of the spin curvature terms. 
TABLE 6.4. (value at $\mathscr{E}=-\frac{m-1}{2} J$ ) minus (value at $\mathscr{E}=-\frac{m-2}{2} J$ ), untraced

\begin{tabular}{|l|c|c|c|c|c|c|}
\hline & $\int|d J|^{2}$ & $\int J^{3}$ & $\int J|V|^{2}$ & $\int \operatorname{tr} V^{3}$ & & coef. \\
\hline $\int|\nabla \mathscr{E}|^{2}$ & $\frac{1}{4}(2 m-3)$ & 0 & 0 & 0 & & -420 \\
\hline $\int\langle\nabla \tau, \nabla \mathscr{E}\rangle$ & $-(m-1)$ & 0 & 0 & 0 & & -168 \\
\hline
\end{tabular}

\begin{tabular}{|l|c|c|c|c|c|}
\hline & $J^{3}$ & $J|V|^{2}$ & $\operatorname{tr} V^{3}$ & & coef. \\
\hline $\mathscr{E}^{3}$ & $-\frac{1}{8}\left(3 m^{2}-9 m+7\right)$ & 0 & 0 & & 840 \\
\hline$\tau \mathscr{E}^{2}$ & $\frac{1}{2}(m-1)(2 m-3)$ & 0 & 0 & & 420 \\
\hline$\tau^{2} \mathscr{E}$ & $-2(m-1)^{2}$ & 0 & 0 & & 70 \\
\hline$|\rho|^{2} \mathscr{E}$ & $-\frac{1}{2}(3 m-4)$ & $-\frac{1}{2}(m-2)^{2}$ & 0 & & -28 \\
\hline$|R|^{2} \mathscr{E}$ & -2 & $-2(m-2)$ & 0 & & 28 \\
\hline
\end{tabular}

New terms in $(4 \pi)^{m / 2} 7 ! 2^{-[m / 2]} a_{6}\left[\not{ }^{2}\right]$ resulting from spinor $(\Sigma)$ bundle curvature $W_{i j}=-\frac{1}{4} R_{k l i j} \gamma_{k} \gamma_{l} \cdot \mathscr{E}=-\frac{m-1}{2} J$

\begin{tabular}{|c|c|c|c|c|c|c}
\hline & $\int|d J|^{2}$ & $\int J^{3}$ & $\int J|V|^{2}$ & $\int \operatorname{tr} V^{3}$ & & coef. \\
\hline $\int \operatorname{tr}_{\Sigma} W_{i j \mid j} W_{i k \mid k}$ & $-\frac{1}{4}(m-1)$ & 0 & 0 & 0 & & -84 \\
\hline
\end{tabular}

\begin{tabular}{|l|c|c|c|c|c|}
\hline & $J^{3}$ & $J|V|^{2}$ & $\operatorname{tr} V^{3}$ & coef. \\
\hline $\operatorname{tr}_{\Sigma} W_{i j} W_{j k} W_{k i}$ & $\frac{1}{4}$ & $\frac{3}{4}(m-3)$ & $-\frac{1}{4}(3 m-8)$ & 56 \\
\hline$\tau \operatorname{tr}_{\Sigma} W_{i j} W_{i j}$ & $-(m-1)$ & $-(m-1)(m-2)$ & 0 & & 70 \\
\hline$\rho_{i j} \operatorname{tr}_{\Sigma} W_{k i} W_{k j}$ & $-\frac{1}{2}$ & $-\frac{5}{4}(m-2)$ & $-\frac{1}{4}(m-2)(m-4)$ & & 56 \\
\hline$R_{i j k l} \operatorname{tr}_{\Sigma} W_{i j} W_{k l}$ & 0 & -3 & $-(m-4)$ & & 28 \\
\hline $\operatorname{tr}_{\Sigma} \mathscr{E} W_{i j} W_{i j}$ & $\frac{1}{4}(m-1)$ & $\frac{1}{4}(m-1)(m-2)$ & 0 & 420 \\
\hline
\end{tabular}


Lemma 6.10.

$$
\begin{aligned}
(4 \pi)^{m / 2} 7 ! 2^{-[m / 2]} a_{6}\left[\nabla^{2}\right]=\int\{ & -3(m-1)(m-6)|\nabla J|^{2} \\
& -\frac{1}{9}\left(35 m^{3}-231 m^{2}+316 m+6\right) J^{3} \\
& +\frac{1}{3}\left(14 m^{3}-39 m^{2}-83 m+24\right) J|V|^{2} \\
& \left.-\frac{2}{9}\left(5 m^{3}+21 m^{2}-95 m-120\right) \operatorname{tr} V^{3}\right\} d v \\
=-\frac{191}{12} \int Q_{6} d v+(m-6) \int & \left\{-\frac{1}{24}(72 m-263)|\nabla J|^{2}\right. \\
& -\frac{1}{144}\left(560 m^{2}-909 m-398\right) J^{3} \\
& +\frac{1}{3}\left(14 m^{2}+45 m-4\right) J|V|^{2} \\
& \left.-\frac{2}{9}\left(5 m^{2}+51 m+211\right) \operatorname{tr} V^{3}\right\} d v .
\end{aligned}
$$

As a result, for $(n, A)=\left(6, \nabla^{2}\right)$ and the choice $u_{\nabla^{2}}(m)=(4 \pi)^{m / 2} 2^{m / 2}(m$ even), the function in 5.1(b) can be taken to be

$$
\alpha_{6}(m)=-\frac{191}{12 \cdot 7 !}
$$

and the local invariant can be taken so that

$$
\begin{aligned}
7 ! B_{6}(m)=- & \frac{1}{24}(72 m-263)|\nabla J|^{2}-\frac{1}{144}\left(560 m^{2}-909 m-398\right) J^{3} \\
& +\frac{1}{3}\left(14 m^{2}+45 m-4\right) J|V|^{2}-\frac{2}{9}\left(5 m^{2}+51 m+211\right) \operatorname{tr} V^{3} .
\end{aligned}
$$

In particular,

$$
\begin{aligned}
& 72 \cdot 7 !\left(\alpha_{6}(6), k_{0}(6), k_{1}(6)-\frac{1}{2} k_{3}(6), k_{2}(6)+\frac{3}{2} k_{3}(6)\right) \\
& \quad=(-1146,-507,-1578,1752) .
\end{aligned}
$$

In the next section we shall show that on $S^{6}$, the quantity $\operatorname{det} Y_{\omega}$ is maximized at those $g=g_{\omega}=e^{2 \omega} g_{0} \in\left[g_{0}\right]$ of volume $16 \pi^{3} / 15$ for which $g_{\omega}=h \cdot g_{0}$ for some $h \in \operatorname{ctran}\left(S^{6}, g_{0}\right)$, and the quantity det $\nabla_{\omega}^{2}$ is minimized at exactly the same metrics. The proof will make essential use of (1) Theorem 3.7 (the exponential class inequality describing the embedding $L_{3}^{2} \hookrightarrow e^{L}$ ); (2) Corollary 3.6(b) (controlling the embedding $L_{1}^{2} \hookrightarrow L^{3}$ ); (3) Obata's Theorem; (4) a geometric inequality describing the embedding $L_{1}^{2}\left(S^{6}, \mathrm{TFS}^{2}\right) \hookrightarrow L^{3}\left(S^{6}, \mathrm{TFS}^{2}\right)$, where TFS $^{2}$ is the bundle of trace-free symmetric tensors; and (5) the numerical data of Lemmas 6.9 and 6.10 .

\section{GeOMETRIC INEQUALITIES IN DIMENSION 6}

Recall from Theorem 6.6 and Lemma 6.9 that if $g_{0}$ is the standard $S^{6}$ metric, then the scale invariant determinant functional for the conformal Laplacian 
in the standard conformal class on $S^{6}$ is

(7.1)

$$
\begin{aligned}
-3 \cdot 7 ! \cdot \frac{1}{2}(4 \pi)^{3} \log \frac{\mathscr{D}\left(Y, e^{2 \omega} g_{0}\right)}{\mathscr{D}\left(Y, g_{0}\right)} & =\frac{64 \pi^{3}}{3} \cdot 10 \mathscr{B}_{6} \\
+\frac{16 \pi^{3}}{15}\left\{1 3 \int _ { S ^ { 6 } } \left\{\left(|d J|^{2} d \xi\right)_{\omega}\right.\right. & \left.-\left(|d J|^{2} d \xi\right)_{0}\right\} \\
& +34 \int_{S^{6}}\left\{\left(J^{3} d \xi\right)_{\omega}-\left(J^{3} d \xi\right)_{0}\right\} \\
& \left.-32 \int_{S^{6}}\left\{\left(J|V|^{2} d \xi\right)_{\omega}-\left(J|V|^{2} d \xi\right)_{0}\right\}\right\},
\end{aligned}
$$

where $\mathscr{B}_{6}$ is the nonnegative quantity estimate by the Beckner-Moser-Trudinger inequality (Theorem 3.7):

$$
\mathscr{B}_{6}:=-\log \int_{S^{6}} e^{6(\omega-\bar{\omega})}(d \xi)_{0}+\frac{1}{40} \int_{S^{6}} \omega\left(\Delta_{0}\left(\Delta_{0}+4\right)\left(\Delta_{0}+6\right) \omega\right)(d \xi)_{0} .
$$

By Lemma 6.10, the corresponding functional for the square of the Dirac operator is

$$
\begin{aligned}
-72 \cdot 7 ! \cdot \frac{1}{2}(4 \pi)^{3} 2^{-3} & \log \frac{\mathscr{D}\left(\mathbb{D}^{2}, e^{2 \omega} g_{0}\right)}{\mathscr{D}\left(\mathbb{X}^{2}, g_{0}\right)}=\frac{64 \pi^{3}}{3} \cdot(-1146)_{\mathscr{B}_{6}} \\
+ & \frac{16 \pi^{3}}{15}\left\{-507 \int_{S^{6}}\left\{\left(|d J|^{2} d \xi\right)_{\omega}-\left(|d J|^{2} d \xi\right)_{0}\right\}\right. \\
& -1578 \int_{S^{6}}\left\{\left(J^{3} d \xi\right)_{\omega}-\left(J^{3} d \xi\right)_{0}\right\} \\
+ & \left.1752 \int_{S^{6}}\left\{\left(J|V|^{2} d \xi\right)_{\omega}-\left(J|V|^{2} d \xi\right)_{0}\right\}\right\} .
\end{aligned}
$$

In each case, we would like to know that the second group of terms on the right, i.e., that with coefficient $16 \pi^{3} / 15$, is sharply estimated in the same way (and with the same sign) as the first $\left(\mathscr{B}_{6}\right)$ term. Remarkably, the coefficients that we have extracted from the heat invariants are such that this is the case:

Theorem 7.1. On $S^{6}$, for $g=g_{\omega}=e^{2 \omega} g_{0} \in\left[g_{0}\right]$ in the conformal class of the standard metric $g_{0}$ and having the standard volume $v_{6}=16 \pi^{3} / 15$, the quantity $\operatorname{det} Y_{\omega}$ is maximized exactly when

$$
g_{\omega}=h \cdot g_{0}, \quad \text { some } h \in \operatorname{ctran}\left(S^{6}, g_{0}\right) .
$$

The quantity det $\nabla_{\omega}^{2}$ is minimized exactly at the same metrics.

Besides Theorem 3.7, the proof will require a clever use of Corollary 3.6, and some further estimates based on Obata's Theorem and the conformal geometry of the bundle TFS $^{2}$ of trace-free symmetric two tensors; these are given in Lemmas 7.2 and 7.3, and Theorem 7.12.

Lemma 7.2. On $S^{m}$ with $m \geq 3$, for $g_{\omega}=e^{2 \omega} g_{0}, \omega \in C^{\infty}\left(S^{m}\right)$,

$$
\int_{S^{m}}\left(|d J|^{2} d \xi\right)_{\omega} \geq 0,
$$


with equality if and only if

$$
g_{\omega}=\alpha^{2} h \cdot g_{0}, \quad \text { some } h \in \operatorname{ctran}\left(S^{m}, g_{0}\right), \alpha \in \mathbb{R}^{+} .
$$

Proof. The inequality is obvious. Equality holds if and only if $J$ is constant; by Obata's Theorem [Ob] this holds if and only if (7.4).

Lemma 7.3. On $S^{6}$, for $g_{\omega}=e^{2 \omega} g_{0}, \omega \in C^{\infty}\left(S^{6}\right)$,

$$
\int_{S^{6}}\left(\left\{|d J|^{2}+2 J^{3}\right\} d \xi\right)_{\omega} \geq 6\left(\int_{S^{6}}\left(|J|^{3} d \xi\right)_{\omega}\right)^{2 / 3} \geq 54,
$$

with $\int_{S^{6}}\left(\left\{|d J|^{2}+2 J^{3}\right\} d \xi\right)_{\omega}=54$ if and only if (7.4) (with $m=6$ ).

Proof. The Yamabe functional in the metric $g_{\omega}$, at a function $u \in C^{\infty}\left(S^{6}\right)$, is

$$
\mathscr{Y}(\omega, u)=\frac{\left((\Delta+2 J)_{\omega} u, u\right)_{L^{2}\left((d \xi)_{\omega}\right)}}{\|u\|_{L^{3}\left((d \xi)_{\omega}\right)}^{2}}
$$

where $u \in C^{\infty}\left(S^{6}\right)$ is not identically zero. By the solution of the Yamabe problem on the sphere and the conformal covariance relation

$$
\mathscr{Y}(\omega+\eta, u)=\mathscr{Y}\left(\omega, e^{2 \eta} u\right)
$$

for the Yamabe functional (which is valid for general $m,\left(M, g_{0}\right)$, and $\omega, \eta \in$ $C^{\infty}(M)$ ), we have

$$
\mathscr{Y}(\omega, u) \geq \mathscr{Y}(0,1)=2 J_{0}=6 .
$$

Applying this to $u=J_{\omega}$, we get the first $\geq$ in (7.5). Corollary 3.6(b) with $m=6, n=2$ gives the second $\geq$, and shows that equality holds there if and only if (7.4). But any $g_{\omega}$ satisfying (7.4) has $J \equiv 3 \alpha^{-2}$ and $\int_{S^{6}}(d \xi)_{\omega}=\alpha^{6}$; showing that $\int_{S^{6}}\left(\left\{|d J|^{2}+2 J^{3}\right\} d \xi\right)_{\omega}=54$ whenever (7.4) holds.

The third inequality is derived using a second order conformally covariant differential operator $D_{(2)}$ on sections of TFS ${ }^{2}$ first constructed by V. Wünsch [Wü, Proposition 3.2]. We shall give a construction of $D_{(2)}$ which is easier to generalize, and is based on the group theoretic machinery we have already put into place. The idea of the corresponding estimate is similar to that of the proof of Lemma 7.3: we look at the quantity $\left(\left(D_{(2)} b, b\right)_{L^{2}\left(S^{6}, T F S^{2}\right)}\right)_{\omega}$, where $b$ is the Einstein tensor. (As usual, the subscript $\omega$ indicates that the quantity is computed in the metric $g_{\omega}=e^{2 \omega} g_{0}$.) Fortunately, a deep analysis of the analogue of the Yamabe functional is not required.

To explain the Wünsch operator, we widen the discussion for the moment to oriented Riemannian manifolds $(M, g)$ of dimension $m \geq 3$. (Orientation is just a convenience, and is not really necessary.) Each irreducible representation $(V, \sigma)$ of the structure group $\mathrm{SO}(m)$ defines an associated vector bundle $\mathbb{V}_{\sigma}=\mathscr{F}_{\mathrm{SO}(m)} \times{ }_{\sigma} V$, where $\mathscr{F}_{\mathrm{SO}(m)}$ is the bundle of oriented orthonormal frames. Because the defining representation $\delta$ of $\mathrm{SO}(m)$ is faithful and gives rise to the cotangent (or tangent) bundle, each $\mathbb{V}_{\sigma}$ can be realized as a tensor bundle, though this realization is not unique. The Levi-Civita covariant derivative $\nabla$ carries sections of $\mathbb{V}_{\sigma}$ to sections of $T^{*} M \otimes \mathbb{V}_{\sigma}$ :

$$
\nabla: C^{\infty}\left(M, \mathbb{V}_{\sigma}\right) \rightarrow C^{\infty}\left(M, T^{*} M \otimes \mathbb{V}_{\sigma}\right) .
$$

The bundle $T^{*} M \otimes \mathbb{V}_{\sigma}$ is generally not irreducible under the structure group $\mathrm{SO}(m)$ (i.e., $\delta \otimes \sigma$ is not an irreducible representation); in fact, it is irreducible 
if and only if $\sigma$ is the trivial one-dimensional representation. We decompose into irreducibles:

$$
\delta \otimes \sigma \simeq \mathrm{SO}(m) \bigoplus_{u=1}^{N_{\sigma}} \mu_{u}
$$

on the level of bundles,

$$
T^{*} M \otimes \mathbb{V}_{\sigma} \simeq \mathrm{SO}(m) \bigoplus_{u=1}^{N_{\sigma}} \mathbb{V}_{\mu_{u}} .
$$

By, e.g., [SW], the summands occur with multiplicity one, so it is legitimate to form the gradients

$$
G_{\sigma \mu_{u}}=G_{u}:=\operatorname{Proj}_{\mathbf{V}_{\mu_{u}}} \nabla: C^{\infty}\left(M, \mathbb{V}_{\sigma}\right) \rightarrow C^{\infty}\left(M, \mathbb{V}_{\mu_{u}}\right)
$$

It is immediate that on $C^{\infty}\left(\mathbb{V}_{\sigma}\right)$, with consistent choices of bundle inner product normalizations,

$$
\nabla=\sum_{u=1}^{N_{\sigma}} G_{u}, \quad \nabla^{*} \nabla=\sum_{u=1}^{N_{\sigma}} G_{u}^{*} G_{u} .
$$

Equivalence classes of irreducible representations $\sigma \in \operatorname{Irr}(\mathrm{SO}(m))$ are parameterized by dominant weights, vectors $\sigma \in \mathbb{Z}^{\ell}, \ell=[m / 2]$, with

$$
\begin{aligned}
& \sigma_{1} \geq \ldots \geq \sigma_{\ell} \geq 0, \quad m \text { odd }, \\
& \sigma_{1} \geq \ldots \geq \sigma_{\ell-1} \geq\left|\sigma_{\ell}\right|, \quad m \text { even. }
\end{aligned}
$$

The dominant weight parameter is the highest weight in the representation; note the customary abuse of notation in using $\sigma$ to denote both the representation and its highest weight. When writing dominant weights, we shall sometimes omit terminal strings of zeroes. Examples are $\sigma=(0)$, the trivial representation; $\sigma=(1)$, the defining representation, and $\sigma=(2)$, the trace free symmetric two-tensor representation.

To determine the target bundles for the gradients in (7.7), we just need to perform the decomposition (7.6). By, e.g., [F, Theorem 3.4], $\mu \in\left\{\mu_{u}\right\}$ if and only if $\mu$ is dominant ((7.9) holds) and either

$$
\mu=\sigma \pm e_{a},
$$

for some $a \in\{1, \ldots, \ell\}$, where $e_{a}$ is the $a^{\text {th }}$ unit vector in $\mathbb{Z}^{\ell}$, or

$$
m \text { is odd, } \sigma_{\ell} \neq 0, \mu=\sigma .
$$

In particular,

$$
(1) \otimes(2) \simeq \mathrm{SO}(m)(1) \oplus(3) \oplus(2,1), \quad m \geq 5 .
$$

Now fix $\sigma=(2)$, so that $\mathbb{V}_{\sigma}=\mathrm{TFS}^{2}$, suppose that $m \geq 5$, and that $(M, g)$ is conformally flat. We are especially interested in computing the effect of the gradients $G_{(1)}, G_{(3)}$, and $G_{(2,1)}$ on the (normalized) Einstein tensor $b=V-(J / m) g$. Because of the identity in Remark 5.4, $V_{i j \mid k}=V_{i k \mid j}$, so

$$
b_{i j \mid k}-b_{i k \mid j}=-\frac{1}{m}\left(J_{\mid k} g_{i j}-J_{\mid j} g_{i k}\right) \text {. }
$$


Note also that

$$
b_{i j \mid j}=\frac{m-1}{m} J_{\mid i} .
$$

Some elementary tensor calculus shows that the (3) part of any three tensor $\left(\varphi_{i j k}\right)$ which is trace free and symmetric in the $j$ and $k$ arguments is

$$
\left(\operatorname{Proj}_{(3)} \varphi\right)_{i j k}=\frac{1}{3}\left(\varphi_{i j k}+\varphi_{j k i}+\varphi_{k i j}\right)-\frac{2}{3(m+2)}\left(g_{i j} \varphi_{l l k}+g_{k i} \varphi_{l l j}+g_{j k} \varphi_{l l i}\right),
$$

and that the (1) part is

$$
-\frac{2}{(m+2)(m-1)} g_{j k} \varphi_{l l i}+\frac{m}{(m+2)(m-1)}\left(g_{i j} \varphi_{l l k}+g_{i k} \varphi_{l l j}\right) .
$$

As a result of these formulas and the identity (7.12),

$$
\begin{aligned}
& \left(\operatorname{Proj}_{(3)} \nabla b\right)_{i j k}=\nabla_{i} b_{j k}+\frac{2}{m(m+2)} J_{\mid i} g_{j k}-\frac{1}{m+2}\left(J_{\mid j} g_{i k}+J_{\mid k} g_{i j}\right), \\
& \left(\operatorname{Proj}_{(1)} \nabla b\right)_{i j k}=-\frac{2}{m(m+2)} J_{\mid i} g_{j k}+\frac{1}{m+2}\left(J_{\mid j} g_{i k}+J_{\mid k} g_{i j}\right) .
\end{aligned}
$$

Adding up, we get

$$
\nabla b=G_{(3)} b+G_{(1)} b ; \quad \text { so } G_{(2,1)} b=0
$$

by (7.8). The interpretation of what has just happened is as follows: if $\mathscr{R}$ is the (reducible) $\mathrm{SO}(m)$ representation carried by tensors of Riemann type, then the multiplicity of $(2,1)$ in $\delta \otimes \mathscr{R}$ is 2 . (See, e.g., [St].) One copy comes from the $\nabla C$ part, and the other from the $\nabla \rho$ part of $\nabla R$. The second Bianchi identity implies that these two are linearly dependent; i.e., that actual Riemann tensors $R$ have their $\nabla R$ living in a bundle with only one $\mathbb{V}_{(2,1)}$ summand. But conformal flatness implies that the expression in $\nabla C$ associated to the first copy vanishes; hence all $(2,1)$ information vanishes.

For the time being, we once again drop the conformal flatness assumption. In [F], Fegan shows that each gradient is conformally covariant, and computes the corresponding conformal bidegrees. Since there may be many different tensor realizations of a $\mathbb{V}_{\sigma}$, each with its own internal conformal weight, it is important to set all internal conformal weights to zero (or some other chosen value) in order to state the result cleanly. The structure group of $m$ dimensional oriented Riemannian conformal geometry is $\mathrm{CO}(m):=\mathbb{R}^{+} \times \mathrm{SO}(m)$; the irreducible representations of $\mathrm{CO}(m)$ are the

$$
\sigma^{p}(a, h)=a^{p} \sigma(h), \quad a \in \mathbb{R}^{+}, h \in \mathrm{SO}(m),
$$

where $p \in \mathbb{C} ; p$ is the internal conformal weight. We let $\mathbb{V}_{\sigma}^{p}$ be the corresponding $\mathrm{CO}(\mathrm{m})$ bundle. The tangent bundle naturally carries the defining representation of $\mathrm{SO}(m)$ with internal conformal weight -1 ; while the cotangent bundle has internal conformal weight +1 . When we realize TFS $^{2}$ as a bundle of covariant tensors (i.e., in the tensor square of $T^{*} M$ ), we give it internal conformal weight +2 ; similarly, we have been computing with a realization of $\mathbb{V}_{(3)}$ that has conformal weight +3 . Because $\nabla g=0$, the construction of $\nabla$, of the $\operatorname{Proj}_{\mu_{u}}$, and of natural differential operators is insensitive to the 
internal conformal weight. The conformal bidegree of a conformally covariant operator, however, is definitely affected by the choice of internal conformal weights. In fact, a chase through the formalism shows that if

$$
D: C^{\infty}\left(M, \mathbb{V}_{\sigma}^{p}\right) \rightarrow C^{\infty}\left(M, \mathbb{V}_{\mu}^{q}\right)
$$

is conformally covariant of bidegree $(a, b)$, then

$$
D: C^{\infty}\left(M, \mathbb{V}_{\sigma}^{p^{\prime}}\right) \rightarrow C^{\infty}\left(M, \mathbb{V}_{\mu}^{q^{\prime}}\right)
$$

is conformally covariant of bidegree $\left(a-p^{\prime}+p, b-q^{\prime}+q\right)$; in particular,

$$
D: C^{\infty}\left(M, \mathbb{V}_{\sigma}^{p+a}\right) \rightarrow C^{\infty}\left(M, \mathbb{V}_{\mu}^{q+b}\right)
$$

is conformally invariant.

Theorem 7.4 [F]. If the gradient $G_{\sigma \mu}$ exists, i.e., if (7.10) or (7.11) holds, then

$$
G_{\sigma \mu}: C^{\infty}\left(M, \mathbb{V}_{\sigma}^{p}\right) \rightarrow C^{\infty}\left(M, \mathbb{V}_{\mu}^{p+1}\right)
$$

is conformally invariant for

$$
p=p_{\sigma \mu}:=\frac{1}{2}\left(m-1+\left\langle 2 \rho_{\mathfrak{s o}(m)}+\sigma+\mu, \sigma-\mu\right\rangle\right),
$$

where $2 \rho_{\mathfrak{s o}(m)}=(m-2, m-4, \ldots, m-2 \ell)$. All first order conformally covariant operators between $\mathrm{SO}(m)$ irreducible tensor bundles arise in this way.

For many values of $\sigma$, Fegan's Theorem leads to a natural construction of a second order conformally invariant operator from $\mathbb{V}_{\sigma}^{(m-2) / 2} \rightarrow \mathbb{V}_{\sigma}^{(m+2) / 2}$; we shall present this construction here. Note that the formal adjoint of a gradient, being conformally covariant, is, up to normalization, another gradient by Theorem 7.4. In the notation of (7.7), consider the compositions

$$
C^{\infty}\left(M, \mathbb{V}_{\sigma}^{(m-2) / 2}\right) \stackrel{G_{u}}{\rightarrow} C^{\infty}\left(M, \mathbb{V}_{\mu_{u}}^{m / 2}\right) \stackrel{G_{u}^{*}}{\rightarrow} C^{\infty}\left(M, \mathbb{V}_{\sigma}^{(m+2) / 2}\right)
$$

(To normalize the formal adjoint construction, take the product metric on the bundle $T^{*} M \otimes \mathbb{V}_{\sigma}$.) Fegan's Theorem shows that, with these choices of internal conformal weights, $G_{u}$ is conformally covariant of bidegree $\left(c_{u}, c_{u}\right)$, and $G_{u}^{*}$ is conformally covariant of bidegree $\left(-c_{u},-c_{u}\right)$, where

$$
c_{u}:=\frac{1}{2}\left(1+\left\langle 2 \rho_{\mathfrak{s o}(m)}+\sigma+\mu_{u}, \sigma-\mu_{u}\right\rangle\right) .
$$

Thus we have the conformal variational formula

$$
\begin{aligned}
\left.(d / d \varepsilon)\right|_{\varepsilon=0}\left(G_{u}^{*} G_{u}\right)_{\varepsilon \omega} & =c_{u}\left(G_{u}^{*}\left[G_{u}, \mu(\omega)\right]-\left[G_{u}^{*}, \mu(\omega)\right] G_{u}\right) \\
& =c_{u}\left(G_{u}^{*}\left[G_{u}, \mu(\omega)\right]+\left[G_{u}, \mu(\omega)\right]^{*} G_{u}\right) .
\end{aligned}
$$

Since

$$
\sum_{u}\left[G_{u}, \mu(\omega)\right] \varphi=[\nabla, \mu(\omega)] \varphi=d \omega \otimes \varphi=: t(\omega) \varphi
$$

for any smooth section $\varphi$, we find that

$$
\left.(d / d \varepsilon)\right|_{\varepsilon=0} \sum_{u=1}^{N_{\sigma}} c_{u}^{-1}\left(G_{u}^{*} G_{u}\right)_{\varepsilon \omega}=\nabla^{*} t(d \omega)+t(d \omega)^{*} \nabla
$$

where the right side is evaluated in the background metric $g=g_{0}$, provided no $c_{u}$ vanishes. But computing in a local orthonormal frame,

$$
\left(\nabla^{*} t(d \omega)+t(d \omega)^{*} \nabla\right) \varphi=-\nabla_{i}\left(\left(\nabla_{i} \omega\right) \varphi\right)+\left(\nabla_{i} \omega\right) \nabla_{i} \varphi=-\left(\nabla_{i} \nabla_{i} \omega\right) \varphi=(\Delta \omega) \varphi .
$$

On the other hand, multiplication by $J$, viewed as an operator from $\mathbb{V}_{\sigma}^{(m-2) / 2}$ to $\mathbb{V}_{\sigma}^{(m+2) / 2}$, has the conformal variation $\mu(\Delta \omega)$. We have proved: 
Theorem 7.5. In the notation above, if no $c_{u}$ vanishes, then

$$
D_{\sigma}:=J-\sum_{u=1}^{N_{\sigma}} c_{u}^{-1} G_{u}^{*} G_{u}
$$

is conformally invariant as an operator from $\mathbb{V}_{\sigma}^{(m-2) / 2}$ to $\mathbb{V}_{\sigma}^{(m+2) / 2}$.

Of course, there is no guarantee that $D_{\sigma}$ is not zero, or zeroth order. Our immediate interest is the behavior of $D_{(2)}$ in the conformally flat case, and especially on $S^{6}$. What we shall use is the following weak consequence of conformal covariance:

Lemma 7.6. The condition

$$
\left(D_{\sigma} \varphi, \varphi\right)_{L^{2}\left(M, \mathbf{v}_{\sigma}\right)} \geq 0, \quad \text { all } \varphi \in C^{\infty}\left(M, \mathbb{V}_{\sigma}\right)
$$

is conformally invariant: if satisfied in the metric $g_{0}$, it is satisfied at all metrics $e^{2 \omega} g_{0}, \omega \in C^{\infty}(M)$.

Note that the $L^{2}\left(M, \mathbb{V}_{\sigma}\right)$ inner product is naturally associated to the $\mathrm{SO}(m)$ bundle $\mathbb{V}_{\sigma}$; that is, it is independent of the internal conformal weight.

Proof of Lemma 7.6. Computing with internal conformal weight 0 , i.e., in $\mathbb{V}_{\sigma}^{0}$, we have

$$
\begin{aligned}
\left(\left(D_{\sigma}\right)_{\omega} \varphi, \varphi\right)_{L^{2}\left(M, g, \mathbf{v}_{\sigma}^{0}\right)} & =\int_{M}\left\langle\left(D_{\sigma}\right)_{\omega} \varphi, \varphi\right\rangle(d v)_{\omega} \\
& =\int_{M} e^{-(m+2) \omega / 2}\left\langle\left(D_{\sigma}\right)_{0}\left(e^{(m-2) \omega / 2} \varphi\right), \varphi\right\rangle e^{m \omega}(d v)_{0} \\
& =\int_{M}\left\langle\left(D_{\sigma}\right)_{0}\left(e^{(m-2) \omega / 2} \varphi\right), e^{(m-2) \omega / 2} \varphi\right\rangle(d v)_{0} .
\end{aligned}
$$

The fact that the bundle inner product $\langle\cdot, \cdot\rangle$ is conformally invariant is a consequence of having set the internal conformal weight to 0 ; if we set it to another value, all terms in the calculation change, but of course the result is the same. Since $\mu\left(e^{(m-2) \omega / 2}\right)$ is bijective on $C^{\infty}\left(M, \mathbb{V}_{\sigma}\right)$, we have the lemma.

We would now like to establish that $\left(S^{6}, g_{0}\right)$ has property $(7.15)$ with $\sigma=$ (2). For this, we return to the thinking of Section 2, and set up the principal series $\operatorname{Ind}_{P}^{G} \sigma \otimes \lambda \otimes 1$. That is, we replace the trivial representation of $(0)$ of SO $(m)$, which we called 1 in Section 2, by the representation $\sigma$. Vectors in this representation can be viewed as sections of the bundle $\mathbb{V}_{\sigma}$ over $S^{m}$, or as functions $\psi$ on $G$ which are equivariant in the sense that, in the notation of Section 2,

$$
\psi(x \operatorname{man})=a^{-\lambda-\rho} \sigma(m)^{-1} \psi(x)
$$

for all $x \in G, m \in M, a \in A, n \in N$. (In particular, $\rho$ is half the sum of the positive $(\mathfrak{g}, \mathfrak{a})$ roots as in Section 2, as opposed to $\rho_{\mathfrak{s o}(m)}$ above, which is half the sum of the $m$ roots.) The $K$ decomposition of the space $\mathscr{E}\left(S^{m}, \mathbb{V}_{\sigma}\right)$ of $K$-finite sections is given by Frobenius reciprocity, which gives a natural identification of $\operatorname{Hom}_{M}(\lambda, \beta)$ and $\operatorname{Hom}_{K}\left(\mathscr{E}\left(S^{m}, \mathbb{V}_{\sigma}\right), \beta\right)$ for any $\beta \in \operatorname{Irr}(K) \simeq \operatorname{Irr}(\operatorname{SO}(m+1))$. In particular, the multiplicity of a $K$-type $\beta$ in $\mathscr{E}\left(S^{m}, \mathbb{V}_{\sigma}\right)$ is the same as the multiplicity of the $M$-type $\sigma$ in $\left.\beta\right|_{M}$. This latter multiplicity $m(\beta, \sigma)$ is given by the following branching rule. First note 
that the $\beta \in \operatorname{Irr}(K)$ are parameterized by dominant weights as in (7.9), with $m+1$ in place of $m$. By [Bo, p. 143], $m(\beta, \sigma)$ is either 0 or 1 , and is 1 if and only if

$$
\begin{aligned}
& \beta_{1} \geq \sigma_{1} \geq \beta_{2} \geq \sigma_{2} \geq \ldots \geq \sigma_{\ell} \geq\left|\beta_{\ell+1}\right|, \quad m \text { odd }, \\
& \beta_{1} \geq \sigma_{1} \geq \beta_{2} \geq \sigma_{2} \geq \ldots \geq \sigma_{\ell-1} \geq \beta_{\ell} \geq\left|\sigma_{\ell}\right|, \quad m \text { even. }
\end{aligned}
$$

We say that $\beta \downarrow \sigma$ (or $\sigma \uparrow \beta$ ) if (7.16) holds. By the multiplicity one property, the splitting

$$
\mathscr{E}\left(S^{m}, \mathbb{V}_{\sigma}\right) \simeq_{K} \bigoplus_{\beta \downarrow \sigma} \beta
$$

diagonalizes every $K$ invariant differential operator, including $D_{\lambda}$ and the Bochner Laplacian $\nabla^{*} \nabla$. ( $K$ acts by isometries, and all operators which are natural in the sense of Sec. 1 are isometry invariant.) By [Bra3, Theorem 1.1], the eigenvalue of $\nabla^{*} \nabla$ on the $\beta$ summand is

$$
\beta\left(\operatorname{Cas}_{\mathfrak{k}}\right)-\sigma\left(\operatorname{Cas}_{\mathfrak{m}}\right),
$$

where Cas is the Casimir operator;

$$
\beta\left(\operatorname{Cas}_{\mathfrak{k}}\right)=\left\langle 2 \rho_{\mathfrak{k}}+\beta, \beta\right\rangle_{\mathbb{R}^{L}} \quad \sigma\left(\operatorname{Cas}_{\mathfrak{m}}\right)=\left\langle 2 \rho_{\mathfrak{m}}+\sigma, \sigma\right\rangle_{\mathbb{R}^{\ell}},
$$

where $L=[(m+1) / 2]$ and $\rho_{\mathfrak{k}}=(m-1, m-3, \ldots, m+1-2 L)$. Note that $\sigma\left(\mathrm{Cas}_{\mathfrak{m}}\right)$ is just multiplication by a constant on $\mathscr{E}\left(S^{m}, \mathbb{V}_{\sigma}\right)$.

Looking just at $\sigma=(2)$,

$$
\mathscr{E}\left(S^{m}, \mathbb{V}_{(2)}\right)=\bigoplus_{q=0}^{2} \bigoplus_{j=0}^{\infty} F_{(2+j, q)}, \quad m \geq 4,
$$

where

$$
F_{(2+j, q)} \simeq_{K}(2+j, q)
$$

and $\nabla^{*} \nabla$ acts by

$$
2(1+j)+j(m+1+j)+(m-3+q) q
$$

on $F_{(2+j, q)}$. This allows us to run a "spectrum generating" argument as in Sec. 2 to determine the eigenvalues of $D_{(2)}$.

The action of $(\mathfrak{g}, K)$ on $\mathscr{E}\left(S^{m}, \mathbb{V}_{(2)}\right)$ is by $\left(U_{\nu}^{(2)}, u^{(2)}\right)=:\left(U_{\nu}, u\right)$, where $u(h)=h$. for $h$ an isometry of $\left(S^{m}, g_{0}\right)$, and $U_{\nu}(X)=\mathscr{L}_{X}+\left(\nu+\frac{m}{2}\right) \omega_{X}$, where $\mathscr{L}_{X}$ is the Lie derivative, $\omega_{X}$ is as in Section 2 , and $\lambda=\nu \alpha$ for $\alpha$ the positive $(\mathfrak{g}, \mathfrak{a})$ root as in Section 2. (In particular, $\omega_{L_{i, m+1}}$ is the homogeneous coordinate function $y_{i}$.) The effect of the Lie derivative (unlike the covariant derivative) depends on the internal conformal weight $p$; in the expression for $U_{\nu}$ in terms of $\mathscr{L}$ immediately above, we have chosen $p=0$.

Lemma 7.7. If $X \in \mathfrak{s}$ and $\beta=(2+j, q) \in \mathbb{N} \times\{0,1,2\}$, then

$$
\omega_{X} F_{\beta}, \mathscr{L}_{X} F_{\beta} \subset F_{\beta-(1,0)} \oplus F_{\beta+(1,0)} \oplus F_{\beta-(0,1)} \oplus F_{\beta+(0,1)},
$$

where by convention, $F_{(-1, q)}=F_{(j,-1)}=F_{(j, 3)}=0$.

Proof. The maps

$$
X \otimes \varphi \mapsto \omega_{X} \varphi, \quad X \otimes \varphi \mapsto U_{\nu}(X) \varphi
$$


are $K$ equivariant from $\mathfrak{s} \otimes F_{(2+j, q)}$ to $\mathscr{E}\left(S^{m}, \mathbb{V}_{(2)}\right)$, the $K$ module structure on $\mathfrak{s}$ being given by the adjoint representation. Since $(\mathfrak{s}$, ad) is a copy of the defining represention of $\mathrm{SO}(m+1), \mathfrak{s} \otimes F_{(2+j, q)}$ is $K$ isomorphic to the space on the right in (7.18), by the selection rule $(7.10,7.11)$ with $m+1$ in place of $m$, and the result follows.

The analogue of Lemma 2.4 is

Lemma 7.8. For $X \in \mathfrak{s},\left[\nabla^{*} \nabla, \mu\left(\omega_{X}\right)\right]=2 U_{0}(X)$.

Proof. The proof is the same as that of Lemma 2.4 until we reach (2.9), which is replaced by

$$
\left[\nabla^{*} \nabla, \mu\left(y_{0}\right)\right]=\mu\left(\Delta y_{0}\right)+2 \nabla_{Z} .
$$

But if $X$ is a conformal vector field, an easy calculation (based on the relations $\nabla_{X} g=0$ and $\left.\mathscr{L}_{X} g=2 \omega_{X} g\right)$ shows that

$$
\mathscr{L}_{X}=\nabla_{X}+p \omega_{X} \quad \text { on } C^{\infty}\left(M, \mathbb{V}_{\sigma}^{p}\right)
$$

in general. This and the fact that $\Delta y_{0}=m y_{0}$ give

$$
\left[\nabla^{*} \nabla, \mu\left(y_{0}\right)\right]=2 \mathscr{L}_{Z}+m \mu\left(y_{0}\right) \quad \text { on } \mathscr{E}\left(S^{m}, \mathbb{V}_{(2)}^{0}\right)
$$

as desired.

Let us say that $\beta \leftrightarrow \gamma$ if and only if $\gamma$ is a summand in the $K$ decomposition of $\mathfrak{s} \otimes \beta$; Lemma 7.7 describes the $\gamma$ satisfying $\beta \leftrightarrow \gamma$ for fixed $\beta \downarrow$ (2). Since $\mathfrak{s}$ is self-contragredient as a $K$ module, the relation $\beta \leftrightarrow \gamma$ is symmetric. (The symmetry of $\leftrightarrow$ can also be read off from (7.10,7.11) with $m+1$ in place of $m$.) The analogue of Lemma 2.3 is:

Lemma 7.9 (Cocycle irreducibility). If $\beta \leftrightarrow \gamma$ and $\beta, \gamma \downarrow(2)$, then

$$
\operatorname{Proj}_{\gamma} \omega(\mathfrak{s}) F_{\beta}=F_{\gamma} \text {. }
$$

As a result, the orbit $\mathscr{O}(\varphi)$ of any nonzero $\varphi \in F_{\beta}$, for any $\beta \downarrow(2)$, under the joint action of $u(K)$ and $\omega(\mathfrak{s})$, is $\mathscr{E}\left(S^{m}, \mathbb{V}_{(2)}\right)$. In particular, $\mathscr{E}\left(S^{m}, \mathbb{V}_{(2)}\right)$ has no nontrivial invariant subspace under this action.

Proof. In analogy with the proof of Lemma 2.3, let

$$
t_{\beta}^{\gamma}=\left.\sum_{i=0}^{m} \operatorname{Proj}_{\beta} \omega_{X_{i}} \operatorname{Proj}_{\gamma} \omega_{X_{i}}\right|_{\beta}
$$

for all $\beta, \gamma \downarrow(2)$, where $\left\{X_{i}\right\}$ is an orthonormal basis of $\mathfrak{s}$. Here we abuse notation by writing $\left.\right|_{\beta}$ and $\operatorname{Proj}_{\beta}$ instead of $\left.\right|_{F_{\beta}}$ and $\operatorname{Proj}_{F_{\beta}}$. The sum on the right in (7.19) is a $K$-endomorphism of $F_{(2+j, q)}$, and thus $t_{\beta}^{\gamma}$ is a scalar by Schur's Lemma. By (2.8),

$$
\sum_{\{\gamma \mid \gamma \leftrightarrow \beta\}} t_{\beta}^{\gamma}=1
$$


By the trace identity $\operatorname{tr} a b=\operatorname{tr} b a$,

$$
(\operatorname{dim} \beta) t_{\beta}^{\gamma}=(\operatorname{dim} \gamma) t_{\gamma}^{\beta}
$$

whenever $\gamma \downarrow(2)$. Unlike their analogues in the scalar case, (7.20) and (7.21) are not strong enough to compute the $t_{\beta}^{\gamma}$. But we can get the rest of the information we need by "squaring" Lemma 7.8. Specifically, let $\kappa_{\beta}=\left\langle 2 \rho_{\mathfrak{k}}+\beta, \beta\right\rangle_{\mathbb{R}^{L}}$ be the eigenvalue of $\beta\left(\operatorname{Cas}_{\mathfrak{k}}\right)$ on the $\beta$ summand of $\mathscr{E}\left(S^{m}, \mathbb{V}_{(2)}\right)$. If $\beta, \gamma \downarrow \sigma$, Lemma 7.8 shows that if $X \in \mathfrak{s}$, then

$$
\left.\left(\kappa_{\gamma}-\kappa_{\beta}\right) \operatorname{Proj}_{\gamma} \omega_{X}\right|_{\beta}=\left.2 \operatorname{Proj}_{\gamma} U_{0}(X)\right|_{\beta} .
$$

Switching the roles of $\beta$ and $\gamma$ in (7.22), composing with the original form of (7.22), summing over $X$ in an orthonormal basis $\left\{X_{i}\right\}$ for $\mathfrak{s}$, and summing over all $\gamma \leftrightarrow \beta$ for fixed $\beta$, we get

$$
-\sum_{\{\gamma \mid \gamma \leftrightarrow \beta\}}\left(\kappa_{\gamma}-\kappa_{\beta}\right)^{2} t_{\beta}^{\gamma}=\left.4 \sum_{i} U_{0}\left(X_{i}\right)^{2}\right|_{\beta}=4\left(U_{0}\left(\operatorname{Cas}_{\mathfrak{g}}\right)-\beta\left(\operatorname{Cas}_{\mathfrak{k}}\right)\right) .
$$

By, e.g., [Kn, 8.22 and 12.28], taking account of our normalizations, $U_{0}\left(\mathrm{Cas}_{\mathfrak{g}}\right)$ is multiplication by the constant $\sigma\left(\operatorname{Cas}_{\mathfrak{m}}\right)-m^{2} / 4$, where here $\sigma=(2)$, so $\sigma\left(\mathrm{Cas}_{\mathfrak{m}}\right)=2 m$. Thus

$$
\sum_{\{\gamma \mid \gamma \leftrightarrow \beta\}}\left(\kappa_{\gamma}-\kappa_{\beta}\right)^{2} t_{\beta}^{\gamma}=m^{2}-8 m+4 \kappa_{\beta}
$$

If $\beta=(2+j, q)$,

$$
\begin{aligned}
& \kappa_{\beta}=(m+j+1)(j+2)+q(m+q-3), \\
& \kappa_{\beta+(1,0)}-\kappa_{\beta}=m+2 j+4, \\
& \kappa_{\beta+(0,1)}-\kappa_{\beta}=m+2 q-2,
\end{aligned}
$$

and by Weyl's dimension formula (see, e.g., [Wa, Theorem 2.4.1.6]),

$$
\begin{aligned}
& \frac{\operatorname{dim}(\beta+(1,0))}{\operatorname{dim} \beta}=\frac{(m+j+q+1)(j-q+4)(m+j)(m+2 j+5)}{(m+j+q)(j-q+3)(j+4)(m+2 j+3)}, \\
& \frac{\operatorname{dim}(\beta+(0,1))}{\operatorname{dim} \beta}=\frac{(m+j+q+1)(j-q+2)(m+q-3)(m+2 q-1)}{(m+j+q)(j-q+3)(q+1)(m+2 q-3)} .
\end{aligned}
$$

This data and $(7.20,7.21,7.23)$ allows us to compute the $t_{\beta}^{\gamma}$ inductively. The 
result is:

$$
\begin{aligned}
& t_{\beta}^{\beta+(1,0)}=\frac{(j+1)(m+j+3)}{(j+3)(m+2 j+3)} \\
& \left.t_{\beta}^{\beta-(1,0)}=\frac{j(m+j+2)}{(m+j)(m+2 j+3)}\right\} \quad \beta=(2+j, 0) \text {, } \\
& t_{\beta}^{\beta+(0,1)}=\frac{2 m}{(j+3)(m+j)} \\
& t_{\beta}^{\beta+(1,0)}=\frac{(j+1)(m+j)(m+j+3)}{(j+2)(m+j+1)(m+2 j+3)} \\
& t_{\beta}^{\beta-(1,0)}=\frac{j(j+3)(m+j+2)}{(j+2)(m+j+1)(m+2 j+3)} \\
& \left.t_{\beta}^{\beta+(0,1)}=\frac{(m-2)(m+1)}{(m+j+1)(j+2)(m-1)}\right\} \\
& \beta=(2+j, 1) \\
& \left.t_{\beta}^{\beta-(0,1)}=\frac{2 m}{(m+j+1)(j+2)(m-1)}\right) \\
& t_{\beta}^{\beta+(1,0)}=\frac{(m+j)(m+j+3)}{(m+j+2)(m+2 j+3)} \\
& \left.t_{\beta}^{\beta-(1,0)}=\frac{j(j+3)}{(j+1)(m+2 j+3)}\right\} \beta=(2+j, 2) . \\
& t_{\beta}^{\beta-(0,1)}=\frac{2}{(j+1)(m+j+2)}
\end{aligned}
$$

(Recall that we assumed $m \geq 5$.) In particular, none of these numbers vanishes, except those giving $t_{\beta}^{\gamma}$ when it is not the case that $\gamma \downarrow \sigma$. This establishes the lemma.

Corollary 7.10. If $\beta=(2+j, q)$ as above, $\beta \leftrightarrow \gamma \downarrow(2)$, and $X \in \mathfrak{g}$,

$$
\left.\operatorname{Proj}_{\gamma} U_{\nu}(X)\right|_{\beta}=\left.c(\beta, \gamma, \nu) \operatorname{Proj}_{\gamma} \omega_{X}\right|_{\beta},
$$

where $c(\beta, \gamma, \nu)=\frac{1}{2}\left(\kappa_{\gamma}-\kappa_{\beta}+2 \nu\right)$. If $|\nu|<(m-2) / 2$, then $\left.\operatorname{Proj}_{\gamma} U_{\nu}(X)\right|_{\beta}$ $=F_{\gamma}$, and the assertions of Lemma 7.9 hold with $\left(u(K), U_{\nu}(\mathfrak{s})\right)$ in place of $(u(K), \omega(\mathfrak{s}))$.

Proof. For $X \in \mathfrak{k}$, both sides of (7.25) vanish, so we are reduced to $X \in \mathfrak{s}$, for which (7.25) follows from (7.22) and the fact that $U_{\nu}=U_{0}+\nu \omega$. For the rest of the statement, we just have to know that $c(\beta, \gamma, \nu) \neq 0$ for $|\nu|<(m-2) / 2$; this follows from (7.24).

With this irreducibility result in place, we can follow the arguments of Section 2 to generate the spectrum of an intertwining operator $A$ of bidegree $(-1,1)$; this operator will be unique up to a constant factor. Since the Wünsch operator $D_{(2)}$, being conformally covariant of bidegree $((m-2) / 2,(m+2) / 2)$, must in particular be intertwining with bidegree $((m-2) / 2,(m+2) / 2)-(m / 2, m / 2)=$ $(-1,1)$, it will have to coincide with $A$ up to a constant factor $C$. Since we are interested in positivity properties of $D_{(2)}$, we shall need to determine (at least the sign of) $C$. 
Lemma 7.11. For $m \geq 5$, the eigenvalue of $D_{(2)}$ on $F_{(2+j, q)}$ is

$$
\mu_{(2+j, q)}=\frac{(m+4+2 j)(m+2+2 j)(m+2 q-2)(m+2 q-4)}{2(m+2)^{2}(m-4)} .
$$

In particular, $D_{(2)}$ satisfies condition $(7.15)$ on $\left(S^{m}, g_{0}\right)$, and thus on $\left(S^{m}\right.$, $\left.e^{2 \omega} g_{0}\right)$ for each $\omega \in C^{\infty}\left(S^{m}\right)$.

Proof. By Lemma 7.9 and Corollary 7.10, an intertwinor of bidegree $(-1,1)$ has

$$
\left(\kappa_{\gamma}-\kappa_{\beta}-2\right) \mu_{\gamma}=\left(\kappa_{\gamma}-\kappa_{\beta}+2\right) \mu_{\beta}
$$

whenever $\beta \leftrightarrow \gamma$ and $\beta, \gamma \downarrow$ (2). By the proof of Corollary 7.10, all coefficients in this relation are nonzero, so the list $\left\{\mu_{\beta}\right\}$ is uniquely determined up to a constant factor. Computing inductively, we get

$$
\mu_{(2+j, q)}=C \frac{(m+4+2 j)(m+2+2 j)(m+2 q-2)(m+2 q-4)}{(m+4)(m+2)(m-2)(m-4)},
$$

where $C=\mu_{(2,0)}$ is a constant which may depend on $m$. By Theorem 7.5,

$$
D_{(2)}=J+\frac{2}{m+2} G_{(3)}^{*} G_{(3)}-\frac{2}{m+2} G_{(1)}^{*} G_{(1)}+\frac{2}{m-4} G_{(2,1)}^{*} G_{(2,1)} \text {. }
$$

(Note that (7.27) and the conformal covariance relation for $D_{(2)}$ are completely general, and in particular do not depend on conformal flatness of the metric.) By the branching rule (7.16) and Frobenius reciprocity, the multiplicity of $(2,0)$ in the $K$ decomposition of $\mathscr{E}\left(S^{m}, \mathbb{V}_{(3)}\right)$ is 0 , and similarly for $\mathscr{E}\left(S^{m}, \mathbb{V}_{(2,1)}\right)$. Since gradients are $K$ equivariant operators, Schur's Lemma implies that $G_{(3)} \varphi=0$ and $G_{(2,1)} \varphi=0$, so computing in the standard sphere metric $g_{0}$, we get

$$
D_{(2)} \varphi=\left(J-\frac{2}{m+2} G_{(1)}^{*} G_{(1)}\right) \varphi=\left(J-\frac{2}{m+2} \nabla^{*} \nabla\right) \varphi, \quad \varphi \in F_{(2,0)} .
$$

But with $g_{0}$ as the metric, $J=m / 2$, and $\nabla^{*} \nabla \varphi=2 \varphi$ by (7.17). Thus

$$
C=\mu_{(2,0)}=\frac{(m+4)(m-2)}{2(m+2)},
$$

as desired for (7.26). The statement about condition (7.15) follows from Lemma 7.6 .

Theorem 7.12. Suppose $m \geq 5$, and let $g_{0}$ be the standard metric on $S^{m}$. Then on $\left(S^{m}, g_{\omega}\right)$ with $g_{\omega}=e^{2 \omega} g, \omega \in C^{\infty}\left(S^{m}\right)$,

$\int_{S^{m}}\left(\left\{\frac{2(m-1)(m-2)}{m(m+2)^{2}}|d J|^{2}-\frac{1}{m} J^{3}+\frac{m+4}{m+2} J|V|^{2}-\frac{2 m}{m+2} \operatorname{tr} V^{3}\right\} d \xi\right)_{\omega} \geq 0$ with equality if and only if (7.4) holds. If $m=6$,

$$
\int_{S^{6}}\left(\left\{|d J|^{2}+\frac{28}{5} J^{3}-\frac{48}{5} J|V|^{2}\right\} d \xi\right)_{\omega} \geq 108,
$$

with equality if and only if (7.4) holds (with $m=6$ ).

Proof. By (7.14) and (7.27), on any Riemannian manifold $(M, g)$,

$$
\begin{aligned}
D_{(2)} b & =\left(J+\frac{2}{m+2} G_{(3)}^{*} G_{(3)}-\frac{2}{m+2} G_{(1)}^{*} G_{(1)}\right) b \\
& =\left(J+\frac{2}{m+2} \nabla^{*} \nabla-\frac{4}{m+2} G_{(1)}^{*} G_{(1)}\right) b .
\end{aligned}
$$


Thus

$$
\left(D_{(2)} b, b\right)_{L^{2}\left(M, g, \mathrm{TFS}^{2}\right)}=\int_{M} J|b|^{2} d v+\frac{2}{m+2}\|\nabla b\|^{2}-\frac{4}{m+2}\left\|G_{(1)} b\right\|^{2},
$$

where the norms are those of the $L^{2}$ section spaces of the appropriate bundles. But

$$
\|\nabla b\|^{2}=\left\|\nabla V-\frac{1}{m} d J \otimes g\right\|^{2}=\|\nabla V\|^{2}-\frac{1}{m}\|d J\|^{2} .
$$

At this point we restrict to the conformally flat case. Using Remark 5.4 and integrating by parts, we get

$$
\|\nabla V\|^{2}=\int V_{i j \mid k} V_{i j \mid k} d v=\int V_{i j \mid k} V_{i k \mid j} d v=-\int V_{i j \mid k j} V_{i k} d v .
$$

By the Ricci and Bianchi identities,

$$
\begin{aligned}
V_{i j \mid k j} & =V_{i j \mid j k}+R_{p i k j} V_{p j}-R_{j p k j} V_{i p} \\
& =J_{\mid i k}-R_{i j k l} V_{j l}+\rho_{p k} V_{i p} .
\end{aligned}
$$

But

$$
\rho_{p k} V_{i p}=(m-2) V_{p k} V_{i p}+J V_{i k},
$$

and (using conformal flatness again),

$$
-R_{i j k l} V_{j l}=V_{i l} V_{k l}-J V_{i k}+V_{j k} V_{j i}-|V|^{2} g_{i k} \text {. }
$$

Furthermore, integrating by parts again and using the Bianchi identity,

$$
\int J_{\mid i k} V_{i k} d v=-\int J_{\mid i} V_{i k \mid k} d v=-\int|d J|^{2} d v
$$

Collecting calculations, we get that

$$
\|\nabla V\|^{2}=\int|d J|^{2} d v+\int J|V|^{2} d v-m \int \operatorname{tr} V^{3} d v
$$

in the conformally flat case. By (7.29),

$$
\|\nabla b\|^{2}=\frac{m-1}{m} \int|d J|^{2} d v+\int J|V|^{2} d v-m \int \operatorname{tr} V^{3} d v
$$

By (7.13),

$$
\left\|G_{(1)} b\right\|^{2}=\frac{2(m-1)}{m(m+2)} \int|d J|^{2} d v
$$

Finally,

$$
J|b|^{2}=J|V|^{2}-\frac{1}{m} J^{3}
$$

Collecting the information from (7.28), (7.30), (7.31), and (7.32), we get that

$$
\begin{aligned}
& \left(D_{(2)} b, b\right)_{L^{2}\left(M, g, \mathrm{TFS}^{2}\right)} \\
& \quad=\int\left\{\frac{2(m-1)(m-2)}{m(m+2)^{2}}|d J|^{2}-\frac{1}{m} J^{3}+\frac{m+4}{m+2} J|V|^{2}-\frac{2 m}{m+2} \operatorname{tr} V^{3}\right\} d v
\end{aligned}
$$

in the conformally flat case. This and Lemma 7.11 imply the first (general $m$ ) inequality in the theorem. Furthermore, by Lemma 7.11 and the conformal covariance relation for $D_{(2)}$, equality holds in this inequality if and only if 
$e^{(m-2) \omega / 2} b_{\omega}$ vanishes, if and only if $b_{\omega}$ vanishes, if and only if $g_{\omega}$ is Einstein. Since Einstein metrics have constant scalar curvature for $m>2$, Obata's Theorem finishes the general $m$ part of the theorem.

Specializing to $m=6$ and multiplying both sides of the inequality by $48 / 5$, we get that

$$
\int_{S^{6}}\left(\left\{|d J|^{2}-\frac{8}{5} J^{3}+12 J|V|^{2}-\frac{72}{5} \operatorname{tr} V^{3}\right\} d \xi\right)_{\omega} \geq 0,
$$

with equality if and only if (7.4) holds. We now use the conformal invariance of $\int Q_{6} d v$ to eliminate the $\operatorname{tr} V^{3}$ term from this inequality: by Theorem 6.4,

$$
\int Q_{6} d v=8 \int\left\{J^{3}-3 J|V|^{2}+2 \operatorname{tr} V^{3}\right\} d v, \quad m=6 .
$$

Adding 0 to both sides of our inequality in the form $\frac{9}{10} \int_{S^{6}}\left\{\left(Q_{6} d v\right)_{\omega}-\left(Q_{6} d v\right)_{0}\right\}$ we get the second $(m=6)$ part of the theorem.

We are now ready to prove the main theorem of this section.

Proof of Theorem 7.1. Neither $Y_{0}$ nor $\nabla_{0}^{2}$ has negative eigenvalues, so the same holds for $Y_{\omega}$ and $\nabla_{\omega}^{2}$ by Theorem 4.8. (Alternatively, the fact that $\not$ is formally self-adjoint implies that $\nabla^{2}$ will never have negative eigenvalues.) Thus maximizing det $Y_{\omega}$ is the same as maximizing

$$
\log \left(\mathscr{D}\left(Y, e^{2 \omega} g_{0}\right)\right) /\left(\mathscr{D}\left(Y, g_{0}\right)\right)
$$

and similarly for minimizing det $\not_{\omega}^{2}$. Collecting the information from (7.1), (7.2), Lemmas 7.2 and 7.3, and Theorem 7.12, and representing the quantity

$$
\begin{aligned}
\int_{S^{6}}\left\{\left(|d J|^{2} d \xi\right)_{\omega}-\left(|d J|^{2} d \xi\right)_{0}\right\} & +b \int_{S^{6}}\left\{\left(J^{3} d \xi\right)_{\omega}-\left(J^{3} d \xi\right)_{0}\right\} \\
& +c \int_{S^{6}}\left\{\left(J|V|^{2} d \xi\right)_{\omega}-\left(J|V|^{2} d \xi\right)_{0}\right\}
\end{aligned}
$$

by the triple $(a, b, c) \in \mathbb{R}^{3}$, we just have to show that the vectors

$$
w_{Y}=:(13,34,-32), \quad-w_{\nabla^{2}}:=(507,1578,-1752)
$$

have positive coefficients when expressed as linear combinations of

$$
u_{1}:=(1,0,0), \quad u_{2}:=(1,2,0), \quad u_{3}:=\left(1, \frac{28}{5},-\frac{48}{5}\right) .
$$

But

$$
3 w_{Y}=6 u_{1}+23 u_{2}+10 u_{3}
$$

and

$$
-2 w_{\not \nabla^{2}}=93 u_{1}+556 u_{2}+365 u_{3} .
$$

Remark 7.13. It is remarkable, and somewhat mysterious, that the vectors (7.33) and (7.34) representing the "trailing terms" of the functional determinants of $Y$ and $\not \nabla^{2}$ fall into the rather narrow positive cone $\mathscr{K}$ generated by $u_{1}, u_{2}$, and $u_{3}$. The coefficients involved are simply buried somewhere in the heat invariants, and thus are determined "purely algebraically." There would seem to 
be no a priori reason that these coefficients should satisfy inequality constraints, but they do. Such "luck" was also present in the four-dimensional case; for each operator, the sign of one constant had to be correct to get an extremal result. Specifically, the quantity asserted to be positive by the norm computation for the Sobolev embedding $L_{1}^{2} \hookrightarrow L^{4}$ had to have a coefficient sign "matching" that of the $L_{2}^{2} \hookrightarrow e^{L}$ quantity. In six dimensions, our proof demanded that three signs fall into place. (The true maximal positive cone is actually larger than the one we have constructed; see the next remark.) The inequalities we have used can be thought of as (at least consequences of) two embeddings $L_{1}^{2} \hookrightarrow L^{3}$, that for scalar functions and that for trace free symmetric two-tensors, and of course $L_{3}^{2} \hookrightarrow e^{L}$. One of the challenges for future work is to explain why these sign miracles occur.

Remark 7.14. It would save quite a bit of delicate conformal geometry, as well as the introduction of the principal series $\operatorname{Ind}_{M A N}^{G}(2) \otimes \nu \otimes 1$, if we could replace the positive quantity $\left(D_{(2)} b, b\right)_{L^{2}\left(S^{6}, g_{\omega}, \mathrm{TFS}^{2}\right)}$ of Theorem 7.12 with the simpler quantity $\left\|G_{(3)} b\right\|^{2}$, which we have not yet used. By (7.8), (7.14), (7.30), and (7.31),

$$
\left\|G_{(3)} b\right\|^{2}=\int\left\{\frac{m-1}{m+2}|d J|^{2}+J|V|^{2}-m \operatorname{tr} V^{3}\right\} d v, \quad m \geq 5 .
$$

Thus

$$
\int\left\{|d J|^{2}+\frac{8}{5} J|V|^{2}-\frac{48}{5} \operatorname{tr} V^{3}\right\} d v \geq 0, \quad m=6 .
$$

Adding 0 in the form $\frac{3}{5} \int_{S^{6}}\left\{\left(Q_{6} d v\right)_{\omega}-\left(Q_{6} d v\right)_{0}\right\}$, we eliminate the $\operatorname{tr} V^{3}$ term as usual; the result is

$$
\int\left\{|d J|^{2}+\frac{24}{5} J^{3}-\frac{64}{5} J|V|^{2}\right\} d v \geq 0, \quad m=6 .
$$

Even on $S^{6}$, this is new information, since the vector

$$
u_{4}:=\left(1, \frac{24}{5},-\frac{64}{5}\right)
$$

representing this quantity is not in the positive cone $\mathscr{K}$ described in the last remark:

$$
3 u_{4}=3 u_{1}-4 u_{2}+4 u_{3},
$$

and of course $u_{1}, u_{2}$, and $u_{3}$ are linearly independent. However, we cannot prove positivity of $w_{Y}$ or of $w_{\nabla^{2}}$ using only $u_{1}, u_{2}$, and $u_{4}$, since

$$
2 w_{Y}=-u_{1}+22 u_{2}+5 u_{4}, \quad-8 w_{\not 2}=-723 u_{1}+3684 u_{2}+1095 u_{4},
$$

and $u_{1}, u_{2}$, and $u_{4}$ are linearly independent.

\section{EPILOGUE}

While signs of trailing terms must miraculously fall into place in order to produce the extremal behavior summarized in Table 0.1 , the sign of the leading term's coefficient is predictable in arbitrary even dimension $m$. Indeed, by [BGØ1, Theorem 1.5], any natural operator of the form $A=\nabla^{*} \nabla-c_{A} J, c_{A}$ 
constant, has

$$
\begin{aligned}
& \left.(4 \pi)^{m / 2} \text { (leading term }\right)\left(U_{m}[A]\right) \\
& \quad=\frac{(-1)^{m / 2}(m / 2) !}{2(m+1) !}\left(-2 m(m-1)+4(m+1) c_{A}\right) \Delta^{(m-2) / 2} J .
\end{aligned}
$$

To be precise about what is meant by "leading term", recall the notation of Corollary 1.5: the right side of $(8.1)$ agrees with $(4 \pi)^{m / 2} U_{m}[A]$ modulo $\mathscr{I}_{m, m-4}$. By Corollary 1.5 , it also agrees with $Q_{m}$ modulo $\mathscr{I}_{m, m-4}$. This in some sense explains the sign pattern in Table $0.1: Y$ and $\nabla^{2}$ both have the form $\nabla^{*} \nabla-c J$, with $c_{Y}=(m-2) / 2, c_{\nabla^{2}}=(m-1) / 2$. But the sign of the quantity $-2 m(m-1)+4(m+1) c_{A}$ changes as $c_{A}$ moves between these two values:

$-2 m(m-1)+4(m+1) c_{Y}=-4, \quad-2 m(m-1)+4(m+1) c_{\nabla^{2}}=2(m-1)$.

Thus

$$
\begin{aligned}
& \text { (leading term })\left(U_{m}[Y]\right)=-(-1)^{m / 2} k_{Y} Q_{m}, \\
& \text { (leading term })\left(U_{m}\left[\nabla^{2}\right]\right)=(-1)^{m / 2} k_{\nabla^{2}} Q_{m},
\end{aligned}
$$

where $k_{Y}$ and $k_{\not{ }^{2}}$ are positive constants. If $U_{m}[A]$ consisted only of its $Q_{m}$ term, the consequence would be the obvious extension of Table 0.1 . Guessing that the signs of the trailing terms' coefficients will continue to fall into place as we go up in dimension, we offer:

Conjecture 8.1. On $S^{m}$ for $m$ even, for $g=g_{\omega}=e^{2 \omega} g_{0} \in\left[g_{0}\right]$ in the conformal class of the standard metric $g_{0}$ and having the standard volume $v_{m}=$ $(4 \pi)^{m / 2} \Gamma(m / 2) / \Gamma(m)$, the quantities $-(-1)^{m / 2} \operatorname{det} Y_{\omega}$ and $(-1)^{m / 2} \operatorname{det} \nabla_{\omega}^{2}$ are maximized exactly when

$$
g_{\omega}=h \cdot g_{0}, \quad \text { some } h \in \operatorname{ctran}\left(S^{m}, g_{0}\right) .
$$

It remains to treat other operators $A$ satisfying our assumptions $4.1,4.3$, and 4.6. For example, limiting ourselves just to operators that we have discussed here, we could treat the $P_{n}$ when (1.6) is satisfied, or the Wünsch operator $D_{(2)}$ of Section 7 when $m \geq 5$. Certainly computations with $P=P_{4}$ in dimension $m=4$ are feasible; the relevant information about the heat invariants is contained in [G3]. Perhaps some analogue of Conjecture 8.1 is more tractable for the operator $P_{m}$, which is in some ways more like the two-dimensional Laplacian than is the conformal Laplacian in dimension $m$.

Another exciting challenge is that of determinants of elliptic boundary value problems. There has been much recent progress in developing computation schemes for boundary contributions to the heat invariants [BG1], and in deriving sharp boundary value Sobolev inequalities [E1-2]. A rich supply of conformally covariant boundary value problems certainly exists; for example, if the basic operator (acting in the interior of our manifold with boundary) is the conformal Laplacian $Y$, one can choose either a pure Dirichlet condition, or a generalized Neumann condition known to physicists as the Robin condition, and have conformal covariance as well as ellipticity. See [E1, Section 1] for a description of the Robin condition, and [BG2] for determinant computations in dimension 4 based on these conditions, as well as for a discussion of the "correct" boundary conditions for a higher order conformal covariant like $P_{n}$. 
In another direction, it should be possible to attack all of these problems (covariant operators, sharp inequalities, functional determinants) in geometries other than Riemannian conformal geometry; specifically, a target of interest and opportunity is CR (tangential Cauchy-Riemann) geometry. Indeed, just as our considerations here are intimately related to the complementary series of representations of $\mathrm{SO}_{0}(m+1,1)$, so are those for CR geometry to the complementary series of $\mathrm{SU}(m+1,1)$. The necessary programs in these other geometries are certainly at a much more primitive state of development than their Riemannian conformal analogues, but the requisite hard analysis and heat operator theory are in progress.

We have concentrated here on finding a (one-sided) bound for the functional determinant. The other side of the coin is the prospect of using the functional determinant to bound other quantities, notably the metric. This brings us into the realm of isospectral problems; specifically, problems of determining the "size" of a set of metrics which are isospectral for some given operator, for example $\Delta$ or $Y$. In this context, "compact in the $C^{\infty}$ topology modulo diffeomorphisms" is the usually conjectured size; if it is possible to show that isospectral classes are singletons (modulo diffeomorphisms), one is "hearing the shape of a drum." The point is that the functional determinant has been crucial in some such investigations, in particular [OPS2] in dimension 2, and [BCY] in dimension 4 . The exponential class inequality needed, again the description of the norm of the embedding $L_{m / 2}^{2} \hookrightarrow e^{L}$, is provided by a transplantation to "bumpy" manifolds of an exponential class inequality of Adams [A], originally proved for domains in $\mathbb{R}^{m}$; see [BCY, Proposition 2.2] for such a transplantation.

Finally, because of our interest in extremal problems on the sphere, we have worked in this paper mostly on the conformally flat case, obtaining general formulas for the functional determinant that involve the Euler characteristic $\chi(M)$. Beyond conformal flatness, $\chi(M)$ tends to get replaced by linear combinations of $\chi(M)$ and various invariants of conformal, but not of topological, structure [BØ3, BCY]. Since there certainly exist manifolds with conformally curved background metrics which exhibit a high degree of symmetry (see, e.g., [BCY, Section 3]), there are new frontiers for the present techniques even within Riemannian conformal geometry.

\section{REFERENCES}

[A] D. Adams, A sharp inequality of J. Moser for higher order derivatives, Ann. of Math. 128 (1988), 385-398.

[BFG] M. Beals, C. Fefferman, and R. Grossman, Strictly pseudoconvex domains in $\mathbb{C}^{n}$, Bull. Amer. Math. Soc. 8 (1983), 125-322.

[Bec] W. Beckner, Sharp Sobolev inequalities on the sphere and the Moser-Trudinger inequality, Ann. of Math. 138 (1993), 213-242.

[Bes] A. Besse, Einstein manifolds, Ergebnisse der Mathematik und ihrer Grenzgebiete, 3. Folge, Band 10, Springer-Verlag, Berlin, 1987.

[BI] D. Bleecker, Determination of a Riemannian metric from the first variation of its spectrum, Amer. J. Math. 107 (1985), 815-831.

[Bo] H. Boerner, Darstellungen von Gruppen, Springer-Verlag, Berlin, 1955.

[Bra1] T. Branson, Differential operators canonically associated to a conformal structure, Math. Scand. 57 (1985), 293-345. 
[Bra2] _ Group representations arising from Lorentz conformal geometry, J. Funct. Anal. 74 (1987), 199-291.

[Bra3] _ Harmonic analysis in vector bundles associated to the rotation and spin groups, $\mathbf{J}$. Funct. Anal. 106 (1992), 314-328.

[BCY] T. Branson, S.-Y. A. Chang, and P. Yang, Estimates and extremals for zeta function determinants on four-manifolds, Comm. Math. Phys. 149 (1992), 241-262.

[BG1] T. Branson and P. Gilkey, The asymptotics of the Laplacian on a manifold with boundary, Comm. Partial Differential Equations 15 (1990), 245-272.

[BG2] _ The functional determinant of a four-dimensional boundary value problem, Trans. Amer. Math. Soc. 344 (1994), 479-531.

[BGØ1] T. Branson, P. Gilkey, and B. Orsted, Leading terms in the heat invariants, Proc. Amer. Math. Soc. 109 (1990), 437-450.

[BGØ2] _ Leading terms in the heat invariants for the Laplacians of the de Rham, signature, and spin complexes, Math. Scand. 66 (1990), 307-319.

[BGP] T. Branson, P. Gilkey, and J. Pohjanpelto, Invariants of locally conformally flat manifolds, Trans. Amer. Math. Soc. 347 (1995), 939-954.

[BØ1] T. Branson and B. Ørsted, Conformal indices of Riemannian manifolds, Compositio Math. 60 (1986), 261-293.

[BØ2] _ Conformal geometry and global invariants, Differential Geom. Appl. 1 (1991), 279308.

[BØ3] _ Explicit functional determinants in four dimensions, Proc. Amer. Math. Soc. 113 (1991), 669-682.

[Bru] F. Bruhat, Sur les représentations induites des groupes de Lie, Bull. Soc. Math. France 84 (1956), 97-205.

[ES] M. Eastwood and M. Singer, A conformally invariant Maxwell gauge, Phys. Lett. 107A (1985), 73-74.

[E1] J. Escobar, Sharp constant in a Sobolev trace inequality, Indiana Univ. Math. J. 37 (1988), 687-698.

[E2] The Yamabe problem on manifolds with boundary, J. Differential Geom. 35 (1992), 21-84.

[F] H. D. Fegan, Conformally invariant first order differential operators, Quart. J. Math. Oxford 27 (1976), 371-378.

[FG] H. D. Fegan and P. Gilkey, Invariants of the heat equation, Pacific J. Math. 117 (1985), 233-254.

[G1] P. Gilkey, Smooth invariants of a Riemannian manifold, Adv. in Math. 28 (1978), 1-10.

[G2] _ Recursion relations and the asymptotic behavior of the eigenvalues of the Laplacian, Compositio Math. 38 (1979), 201-240.

[G3] The spectral geometry of the higher order Laplacian, Duke Math. J. 47 (1980), 511-528.

[G4] Invariance theory, the heat equation, and the Atiyah-Singer Index Theorem, Publish or Perish, Wilmington, DE, 1984.

[G5] L Leading terms in asymptotics of the heat equation, Contemp. Math. 73 (1988), 79-85.

[GJMS] C. R. Graham, R. Jenne, L. Mason, and G. Sparling, Conformally invariant powers of the Laplacian, I: existence, J. London. Math. Soc. 46 (1992), 557-565.

[KI] F. Klein, Vorlesungen über Höhere Geometrie, Springer-Verlag, Berlin, 1926.

[Kn] A. Knapp, Representation theory of semisimple groups, Princeton Univ. Press, Princeton, NJ, 1986.

[Ko] Y. Kosmann, Dérivées de Lie des spineurs, Ann. Mat. Pura Appl. (4) 91 (1972), 317-395.

[Lic] A. Lichnerowicz, Spineurs harmoniques, C. R. Acad. Sci. Paris 257 (1963), 7-9.

[Lie] E. Lieb, Sharp constants in the Hardy-Littlewood-Sobolev and related inequalities, Ann. of Math. 118 (1983), 349-374. 
[M] V. Molčanov, Representations of pseudo-orthogonal groups associated with a cone, Math. USSR-Sb. 10 (1970), 333-347.

[Ob] Obata, The conjectures on conformal transformations of Riemannian manifolds, J. Differential Geom. 6 (1971), 247-258.

[On] E. Onofri, On the positivity of the effective action in a theory of random surfaces, Comm. Math. Phys. 86 (1982), 321-326.

[OPS1] B. Osgood, R. Phillips, and P. Sarnak, Extremals of determinants of Laplacians, J. Funct. Anal. 80 (1988), 148-211.

[OPS2] __ Compact isospectral sets of surfaces, J. Funct. Anal. 80 (1988), 212-234.

[P] S. Paneitz, A quartic conformally covariant differential operator for arbitrary pseudoRiemannian manifolds, preprint, 1983.

[Sc] R. Schimming, Lineare Differentialoperatoren zweiter Ordnung mit metrischem Hauptteil und die Methode der Koinzidenzwerte in der Riemannschen Geometrie, Beiträge Anal. 15 (1981), 77-91.

[SW] E. Stein and G. Weiss, Generalization of the Cauchy-Riemann equations and representations of the rotation group, Amer. J. Math. 90 (1968), 163-196.

[St] R. Strichartz, Linear algebra of curvature tensors and their covariant derivatives, Canad. J. Math. 40 (1988), 1105-1143.

[V] D. Vogan, Unitary representations of reductive Lie groups, Ann. of Math. Stud., Princeton Univ. Press, Princeton, NJ, 1987.

[Wa] G. Warner, Harmonic analysis on semi-simple Lie groups I, Springer-Verlag, Berlin, 1972.

[Wü] V. Wünsch, On conformally invariant differential operators, Math. Nachr. 129 (1986), 269-281.

Department of Mathematics, The University of Iowa, Iowa City, Iowa 52242

E-mail address: branson@math.uiowa.edu

Mathematical Institute, Roskilde University, DK-4000 Roskilde, Denmark

SONDERFORSCHUNGSBEREICH 170, BUNSENSTRASSE 3-5, D-3400 GötTINGEN, GERMANY 